\title{
A Deep Representation Learning Framework for Medical Imaging Data Analysis
}

by

\section{PengCheng Xi}

A Dissertation submitted to

the Faculty of Graduate and Postdoctoral Affairs

in partial fulfilment of

the requirements for the degree of

Doctor of Philosophy

Ottawa-Carleton Institute for Electrical and Computer Engineering

Department of Systems and Computer Engineering

Carleton University

Ottawa, Ontario, Canada

May 2020

Copyright (C)

2020 - PengCheng Xi 


\section{Abstract}

This thesis studies representation learning for medical imaging data analysis. We propose a deep learning framework that is composed of data representation and feature learning. The data representation module deals with challenges arising from the need to analyze the various types of data from medical imaging. Examples of such data include 1D physiological signals, 2D high-resolution images and 3D human shape. The framework uses deep convolutional neural networks (CNN) and deep recurrent

neural networks (RNN) for feature learning from the data representations. Through validations, the proposed framework proves effective in learning representations for detecting abnormalities in the physiological signals and the medical images, as well as identifying landmarks from the human shape data.

The framework starts from converting the various types of medical imaging data to $2 \mathrm{D}$ visual representations unanimously. Transfer learning is a technique for addressing data insufficiency problems in deep learning. With this technique, the framework uses deep CNNs pre-trained on large-scale 2D image sets to extract deep features from the data representations. In applications where the medical data are in a sequential form, the framework also integrates deep RNNs to conduct representation learning in spatial, spectral and temporal domains.

We introduce the representation learning framework through selected tasks, including detecting cardiac murmurs from phonocardiograms (PCGs), recognizing masses and calcifications in mammograms, and locating anatomical landmarks on 
3D human surface data. In the detection of cardiac murmurs, we have found a suitable data representation, which is computed from mel spectrograms within specific frequency ranges. Deep feature learning from the representations has achieved an F-score of 0.9767 . When deployed to the detection of abnormalities in mammograms, the framework trains patch CNNs and builds abnormality detectors that outperform traditional approaches by a large margin. When applied to 3D human surface data, the proposed framework demonstrates that the learned representations are invariant to viewpoints. When applied to the surface data for locating landmarks, the framework finds optimal data representations and it has achieved human-level performance.

In summary, we have proposed a deep representation learning framework and proved its effectiveness in medical imaging data analysis. Experimental results indicate that the framework serves as a guidance on effective representation learning for solving other problems from medical imaging data analysis using deep learning. 
To my family. 


\section{Acknowledgments}

First of all, I would like to thank my thesis advisors, Prof. Rafik Goubran and Dr. Chang Shu. I acknowledge them for their guidance, advice and time during this journey. This thesis would not have been possible without their great support.

I appreciate the feedback and comments from my thesis defense committee. In particular, Prof. Farida Cheriet provided constructive comments and suggestions on how to improve my thesis. Prof. Michel Nakhla gave me suggestions on how to introduce the framework to people outside of the field. Prof. Robert Laganière motivated me with challenging questions. Prof. Richard Yu inspired me to frame my work in a broader perspective. Their inputs have helped me to improve my thesis.

I would like to thank my colleagues and friends. When I started doing the study, I received training on medical device research from Prof. Tofy Mussivand at Ottawa Heart Institute. Prof. Xiaodan Zhu gave me advice on integrating deep learning with many applications. I have learned from Dr. Hongyu Guo and Prof. Yifeng Li through multiple technical discussions.

I would also like to thank my managers at National Research Council Canada. Dr. Joel Martin has been very supportive during my Ph.D. study. I also received support from Mr. Louis Borgeat for doing the study.

Last but not least, I am very grateful for my family's constant support over the years. 


\section{Table of Contents}

Abstract $\quad$ ii

Acknowledgments $\quad$ V

Table of Contents $\quad$ vi

List of Tables $\quad$ x

List of Figures $\quad$ xii

1 Introduction $\quad 1$

1.1 Problem Statement . . . . . . . . . . . . . . . . 2

1.2 Motivation ........................... 3

1.3 Proposed Framework . . . . . . . . . . . . . . . 5

1.3.1 Data Representation Module . . . . . . . . . . . . 5

1.3.2 Feature Learning Module . . . . . . . . . . . . . . . . . . 6

1.3.3 Applications to Medical Imaging Data Analysis . . . . . . . . 6

1.4 Contribution and Outline . . . . . . . . . . . . . . . . . . . 7

2 Literature Review $\quad 12$

2.1 Machine Learning . . . . . . . . . . . . . . . . . 12

2.1.1 From Feature Engineering to Architecture Engineering . . . . 13

2.1.2 Deep Learning . . . . . . . . . . . . . . . . . . . . . . . 14 
2.2 Related Work . . . . . . . . . . . . . . . . . . . . . 17

$2.2 .1 \quad$ Feature Engineering $\ldots \ldots \ldots \ldots \ldots$

$2.2 .2 \quad$ Deep CNN models . . . . . . . . . . . . . . . . . . . 18

3 Representation Learning on 1D Physiological Signals 23

3.1 Introduction . . . . . . . . . . . . . . . . . . . . . . . . 24

3.2 Related Work . . . . . . . . . . . . . . . . . . 27

3.3 Methodology using Deep CNNs _. . . . . . . . . . . . 30

3.3 .1 Data Selection . . . . . . . . . . . . . . . . . . . . 30

3.3.2 Data Pre-processing . . . . . . . . . . . . . . . . 30

3.3.3 Data Augmentation . . . . . . . . . . . . . . . . . . . 31

3.3.4 Feature Extraction and Model Building . . . . . . . . . . . 32

3.4 Methodology using Deep Recurrent CNNs _. . . . . . . . . . 33

3.4 .1 Data Selection . . . . . . . . . . . . . . . . . . . . . . 34

3.4.2 Feature Extraction and Model Building . . . . . . . . . . . 34

3.5 Experiments with Deep CNNs . . . . . . . . . . . . . . 36

3.5.1 Comparison on Results from Deep CNN Architectures . . . . 36

3.5.2 Prediction on Spectrograms using AlexNet and ResNet . . . . 40

3.5.3 Prediction on Spectrograms using VGGNet and GoogLeNet . 41

3.5.4 Using CNN as Off-The-Shelf Feature Extractors . . . . . . . 42

3.6 Experiments with Deep Recurrent CNNs . . . . . . . . . . . . . 42

3.6.1 Use of Spectrogram as Data Representation . . . . . . . . . 42

3.6.2 Use of Scalogram and Mel Spectrogram . . . . . . . . . . 45

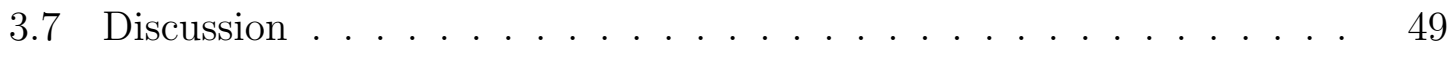

3.8 Conclusion . . . . . . . . . . . . . . . . . . . . . . . . . . . . . 50

4 Representation Learning on 2D Medical Images 51

4.1 Introduction . . . . . . . . . . . . . . . . . . 52 
4.2 Related Work . . . . . . . . . . . . . . . . . . . 55

4.2.1 Traditional CADe Approaches . . . . . . . . . . . . . . 56

4.2 .2 Deep Learning for CADe . . . . . . . . . . . . . . . 57

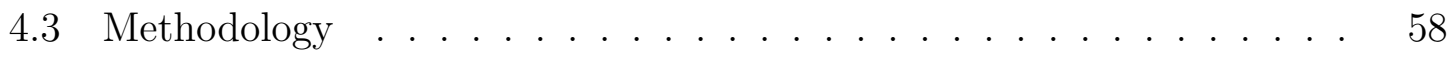

4.3.1 Abnormality Detection with Class Activation Mapping . . . . 60

4.3.2 Abnormality Detection with Faster R-CNN . . . . . . . . 62

4.3.3 Abnormality Detection with YOLO V2 . . . . . . . . 65

4.4 Results . . . . . . . . . . . . . . . . . . . . 66

4.4.1 Abnormality Detection with Class Activation Mapping . . . . 66

4.4.2 Abnormality Detection with Faster R-CNN . . . . . . . . 73

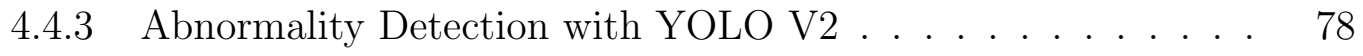

4.5 Discussion . . . . . . . . . . . . . . . . . . . . . . . 85

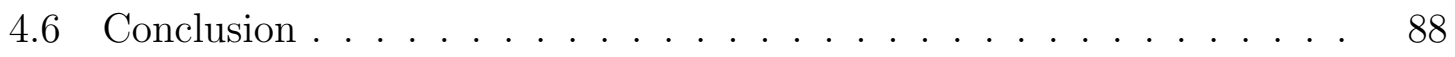

5 Representation Learning on 3D Data for Computing Features $\quad 89$

5.1 Introduction . . . . . . . . . . . . . . . . . . 90

5.2 Related Work . . . . . . . . . . . . . . . . . . . 92

5.3 The Approaches . . . . . . . . . . . . . . . . . . . . . . . 93

5.3 .1 Data Preparation . . . . . . . . . . . . . . . . . 93

$5.3 .2 \quad$ HOG Feature and SVM $\ldots \ldots \ldots \ldots$

5.3.3 Deep Auto-encoders Network . . . . . . . . . . . . . . . 96

5.3.4 Deep Convolutional Neural Network and SVM _ . . . . . . 97

5.3.5 End-to-End Training using Deep CNNs . . . . . . . . . . 97

5.4 Experiments and Implementations . . . . . . . . . . . . . . 98

5.4 .1 Datasets . . . . . . . . . . . . . . . . . . . . . 98

$5.4 .2 \quad$ HOG Feature and SVM $\ldots \ldots \ldots \ldots$

5.4.3 Deep Auto-encoders Network . . . . . . . . . . . . . . . . . . 99 
5.4.4 Deep Convolutional Neural Network and SVM . . . . . . . . 100

5.4.5 End-to-End Training using Deep CNNs . . . . . . . . . . . . 103

5.4.6 Comparison on Performance . . . . . . . . . . . . . . . . 103

5.5 Discussion . . . . . . . . . . . . . . . . . . . . . . . . . . . . . 104

5.6 Conclusion . . . . . . . . . . . . . . . . . . . . . . . . . . . . . . . 105

6 Representation Learning on 3D Data for Locating Landmarks 106

6.1 Introduction . . . . . . . . . . . . . . . . . . . . . . . . . 107

6.1.1 Accurate 3D Landmarking . . . . . . . . . . . . . . . . 108

6.1.2 Deep Convolutional Neural Networks _ . . . . . . . . . . . . 109

6.2 Related Work . . . . . . . . . . . . . . . . . . . 110

6.3 Deep Learning for Landmark Prediction . . . . . . . . . . . . . . 111

6.3 .1 Data Preparation . . . . . . . . . . . . . . . . . . . 112

6.3.2 Deep CNN for Regression . . . . . . . . . . . . . . 113

6.3.3 Deep CNN for Classification . . . . . . . . . . . . . . . . . . 114

6.4 Experimental Results . . . . . . . . . . . . . . . . . . . . . . 114

6.4.1 Data Preparation . . . . . . . . . . . . . . . . . 115

6.4 .2 Deep Regression CNN . . . . . . . . . . . . . . . . 115

6.4.3 Deep Classification CNN . . . . . . . . . . . . . . . 116

6.4.4 Prediction Results . . . . . . . . . . . . . . . . . 117

6.5 Discussion . . . . . . . . . . . . . . . . . . . . . . . . . 121

6.6 Conclusion . . . . . . . . . . . . . . . . . . . . . . . . . . 123

$\begin{array}{lll}7 \text { Conclusion } & 129\end{array}$

List of References 132 


\section{List of Tables}

1 Classification Accuracies of Deep CNNs (step size = 500) . . . . . 36

2 Classification Accuracies of Deep CNNs (step size = 100) . . . . . . 37

3 Classification Accuracies of Deep Recurrent CNNs. . . . . . . . . . . 44

4 Comparison on Classification Accuracy with Traditional Approaches. 45

5 Classification Accuracies (F-Score) using Different Features . . . . . . 46

6 Size of training and testing image patches for CBIS-DDSM mammogram data-set. . . . . . . . . . . . . . . . . . . 67

7 Mean Classification Accuracy of Deep CNNs and SVMs on the Mammogram Data-set . . . . . . . . . . . . . . . . . 69

8 Size of training and testing sets from Nantong data-set of ultrasound liver images. . . . . . . . . . . . . . . . . . . 76

9 Average Precision of Deep Detectors on Different Abnormalities in the Ultrasound Liver Data-set . . . . . . . . . . . . . . . . . . . . . . 78

10 Average Precision of YOLO v2 based Detectors in the Ultrasound Liver Data Set . . . . . . . . . . . . . . . . 79

11 Average Precision of Object Detectors on Mass Detection in the Mammogram Data-set . . . . . . . . . . . . . . . 80

12 Mean Accuracy of Deep CNNs and SVMs on the Liver Data-set . . . 81

13 Composition of the CAESAR dataset. . . . . . . . . . . . . 94 
14 The comparison on classification rates for different dimensions of hidden layers in the auto-encoder networks. The last column lists the number of epochs when reaching minimum gradient during fine-tuning. 99

15 Performance comparison on image classification using different approaches. . . . . . . . . . . . . . . . . . . . 103

16 Results comparison on selected landmarks between the regression network and the classification networks. The numbers in each cell represent the mean and standard deviation of the prediction errors (in pixels) . . . . . . . . . . . . . . . . . . . . . . 121 


\section{List of Figures}

1 The proposed deep representation learning framework for 1D, 2D and 3D medical data analysis. . . . . . . . . . . . . . . . . 4

2 Work flow of deep learning applications. . . . . . . . . . . . . . . . . 14

3 Applying the deep representation learning framework on 1D audio signal data (from left to right: raw data, converted 2D data representations, deep model training and output). . . . . . . . . . . . . . 24

4 PCG recordings and spectrograms computed for (a) a murmur subject and (b) a normal subject. . . . . . . . . . . . . . . . . 31

5 Sample image patches extracted from spectrograms within (a) murmur and (b) normal categories. . . . . . . . . . . . . . . . . . . 32

6 Diagram of a murmur detection approach using deep CNNs. . . . . . 33

7 Diagram of a murmur detection approach using deep recurrent CNNs (FFT - Fast Fourier Transform; FV - Feature Vectors). . . . . . . . . 35

8 Predictions on consecutive image patches extracted from spectrograms computed on (a) a murmur PCG and (b) a normal PCG (step size for training image window $=500)$. From top to bottom: spectrogram, prediction from a AlexNet model, and prediction from a ResNet model. 38 
$9 \quad$ Predictions on consecutive image patches extracted from spectrograms computed on (a) a murmur PCG and (b) a normal PCG (step size for training image window $=100)$. From top to bottom: spectrogram, prediction from a AlexNet model, and prediction from a ResNet model. 39

10 Predictions on consecutive image patches extracted from spectrograms computed on (a) a murmur PCG and (b) a normal PCG (step size for training image window $=100)$. From top to bottom: spectrogram, prediction from a VGGNet model, and prediction from a GoogLeNet model. ........................ . . 41

11 Classification rates (F-score) over different layers in the deep CNN . . 43

12 Different data representations for a murmur case (a, c, e, and g) and a normal case (b, d, f and h). Both cases are three seconds in duration. 47

13 Plot extracted features using spectrograms as input. . . . . . . . . . . 48

14 Applying the deep representation learning framework on 2D medical image data (from left to right: raw data, converted 2D data representations, deep model training and output). . . . . . . . . . .

15 Diagram of an integrated approach for medical abnormality detection using deep convolutional neural networks. . . . . . . . . . . . . . 59

16 Diagram of CAM-based approach: 1) train deep CNNs using abnormality patches; 2) modify network and compute the weights for CAM; 3) given a test image, compute color-coded abnormality map using CAM. 61

17 Diagram of R-CNN-based approach: ResNet is cut at a convolution layer and a region proposal network is added; bounding box classification and regression layers are used to replace the original output layer of the ResNet. . . . . . . . . . . . . . . . . . . . . . 64

18 Accuracy of SVM models trained with features computed from the different layers in the AlexNet (mammogram data-set). . . . . . . . . 70 
19 Calcification localization result (left: full mammogram, middle: class activation map output superimposed on the mammogram, right: ground-truth binary mask image). . . . . . . . . . . . . . . 72

20 Mass localization result (from left to right: full mammogram, class activation map output superimposed on mammogram, ground-truth binary mask image). . . . . . . . . . . . . . . . . .

21 Calcification localization result (left: full mammogram, middle: class activation map superimposed on mammogram, right: ground-truth image). . . . . . . . . . . . . . . . .

22 Mass localization result (left: full mammogram, middle: class activation map superimposed on mammogram, right: ground-truth image). 75

23 Sample images of the CYST data-set (labeled in green rectangles). . . 76

24 Sample images of the PHC data-set (labeled in green rectangles). . . 77

25 Accuracy of SVM models trained with features computed from the different layers in the AlexNet (liver data-set). . . . . . . . . . . . . 81

26 Detection results on two test cases (case 1: (a) - (c) with CYST abnormality; case 2: (d) - (f) with PHC abnormality). Detection results are labeled in yellow with probabilities and ground truth is labeled with green rectangles. . . . . . . . . . . . . . . . . .

27 Detection results on two test cases with CYST abnormality (detection results are labelled in yellow with probabilities, ground truth is labelled with green rectangles. . . . . . . . . . . . . . . . . .

28 Detection results on two test cases with PHC abnormality (detection results are labelled in yellow with probabilities, ground truth is labelled with green rectangles. . . . . . . . . . . . . . . 
29 Applying the deep representation learning framework on 3D human surface data for human recognition (from left to right: raw data, converted 2D data representations, deep model training and output). . .

30 The setup of virtual cameras for capturing full-body images from different viewpoints . . . . . . . . . . . . . . . . . . . . . . . . . . 93

31 Multi-view images rendered from one 3D raw scan (top row: upper front views, bottom row: front views) . . . . . . . . . . . .

32 HOG features computed with different cell sizes on a sample image (top row: sample image, bottom row: computed HOG features (on top of each image icon are the cell size used for computing features and the length of the computed features)) . . . . . . . . . 95

33 A deep neural network using auto-encoders . . . . . . . . . . . . . 96

34 Visualization of features learned by the first encoder. . . . . . . . . . 100

35 Visualization of features learned by the second encoder. . . . . . . . . 101

36 An example selection of first part of the AlexNet (conv1 - pool5) as feature extractor for building an SVM classifier. . . . . . . . . . . . . 101

37 Classification rates over different layers in the deep CNN . . . . . . . 102

38 Applying the deep representation learning framework on 3D human surface data for locating landmarks (from left to right: raw data, converted 2D data representations, deep model training and output). . . 107

39 From left to right: rendered appearance image (eight selected anatomical landmarks are labeled in red), range depth image, and curvature map image. The landmarks are: 1-Sellion 2-Infraorbitale 3Supramenton 4-Suprasternale 5-Thelion 6-Anterior Axilla 7-Radial Styloid and 8-Groin. . . . . . . . . . . . . . . . 113

40 Examples of rendered appearance images. . . . . . . . . . . . . . 116

41 Examples of range depth images. . . . . . . . . . . . . 117 
42 Examples of curvature mapped images. . . . . . . . . . . . . . 118

43 Phantom landmarks selection (red cross (5): current sellion landmark; red square: image patch extraction area around current sellion landmark; blue crosses (1-4): phantom landmarks on four corners; blue square: image patch extraction area around the first phantom landmark) . . . . . . . . . . . . . . . . . . . . 119

44 Comparison on mean and standard deviation of prediction errors on each landmark. . . . . . . . . . . . . . . . . . . . 120

45 Regression result on sample test images (red cross: locations of predicted landmarks from the deep regression CNN. . . . . . . . . . . . 124

46 Window-sweeping image patch classification results for Sellion landmark.125

47 Window-sweeping image patch classification results for Infraorbitale landmark. . . . . . . . . . . . . . . . . . 125

48 Window-sweeping image patch classification results for Supramenton landmark. . . . . . . . . . . . . . . . . . . . . 126

49 Window-sweeping image patch classification results for Suprasternale landmark. . . . . . . . . . . . . . . . . . . 126

50 Window-sweeping image patch classification results for Thelion land-

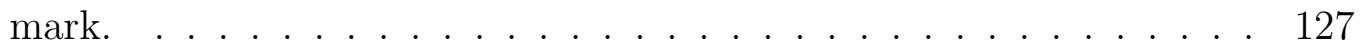

51 Window-sweeping image patch classification results for Anterior Axilla landmark. . . . . . . . . . . . . . . . . . . . . . 127

52 Window-sweeping image patch classification results for Radial Styloid landmark. . . . . . . . . . . . . . . . . . . . . . 128

53 Window-sweeping image patch classification results for Groin landmark.128 


\section{Chapter 1}

\section{Introduction}

The goal of this thesis is to conduct representation learning for medical imaging data analysis. The performance of machine learning algorithms is dependent on the choice of data representation and feature learning [1]. Data representation converts raw data to new forms in order to highlight information in the data. Feature learning computes features from the data representation [1]. Deep learning (DL) is effective in feature learning but does not deal with data representation. For medical imaging data analysis, representation learning remains a challenging research topic.

In this thesis, a deep representation learning framework is proposed for medical imaging data analysis. The framework is composed of data representation and feature learning. The data representation module deals with the need to extract information from the various types of data in medical applications. Examples of the data include physiological signals, high-resolution images and human surface data. The data representation module converts the raw data unanimously to $2 \mathrm{D}$ visual representations. The framework then uses modified convolutional neural networks (CNN) and recurrent neural networks (RNN) for feature learning from the visual representations. Through a series of validations, the framework proves effective in learning deep representations for medical imaging data analysis. 


\section{$1.1 \quad$ Problem Statement}

When applying deep learning to medical imaging data analysis, one faces three major challenges. First, collecting and annotating medical imaging data are costly because it needs expertise from medical professionals. The second challenge comes from the multi-dimensional nature of the data collected in medical applications. For example, electrocardiogram (ECG) and phonocardiogram (PCG) are 1D physiological signals recorded on patients for monitoring their health conditions. X-rays and ultrasound are $2 \mathrm{D}$ images that provide visual information for diagnosis. Doctors also use 3D surface imaging for the planning of operations and computer-aided surgeries.

The third challenge comes from the need to make proper selection or optimal design of deep neural networks for processing the data in different dimensions and types. It is known that the evolving architecture of deep learning models has played an important role in achieving top performance on tasks within ImageNet challenges [2]; however, these models were tailored for general image data sets and it is difficult to tailor models for medical images due to the limited data size. Achieving the top performance also comes at a price: designing the CNN models needs expertise and involves a lot of trials and errors. For medical image analysis, it is difficult to collect training data in the size of ImageNet or to find deep learning models tailor designed for medical imaging data.

Representation learning is about extracting features from data representations in order to extract useful information for building classifiers or predictors [1]. Applying deep learning to medical imaging data requires effective representation learning, which comprises optimal data representations and improved neural network architectures. The two essential components are worth studying in order to make progress towards artificial intelligence (AI) [1]. 


\subsection{Motivation}

Vision is a major source of information in human perception as eighty percent of the perception is estimated to be mediated through the vision [3]. For object recognition, the human vision system follows a hierarchical and feed-forward model [4]. In higher visual areas, receptive fields are larger and neurons are sensitive to complex features such as shapes and objects, whereas in lower visual areas, neurons are sensitive to low-level features, such as edges and lines.

The design of existing deep learning models, especially deep CNNs, has a root in the human vision system. They extract visual features in a similar way as the human vision system. In particular, the CNN models share two common properties with the human visual processing system. First, the receptive field size of individual neurons grows towards the end of a deep CNN model. This is achieved through pooling layers, which help to enlarge the receptive field size for convolutional filters at deeper layers. Second, convolutional layer extracts basic-level features from the shallow side and object-level ones from the deep side of the model [5].

The first notable success of deep CNNs was on improving the accuracy of image classifications in ImageNet. In 2012, AlexNet [6] achieved top-5 test error rate of $15.3 \%$, compared to $26.2 \%$ achieved by the second-best entry. Since then, a series of deep CNN models have been designed to improve the performance on both image classification and object recognition [7-9] and the list keeps growing.

The analysis of medical imaging data lacks two key contributing factors that led to the success of deep learning on ImageNet, namely large amount of training data and improved neural network architectures; therefore, it is worth studying the ways of leveraging the plethora of deep models pre-trained on ImageNet. Fortunately, studies have proven the effectiveness of transfer learning using deep neural networks when target data are not too different from source data [10]. This motivates us to study 


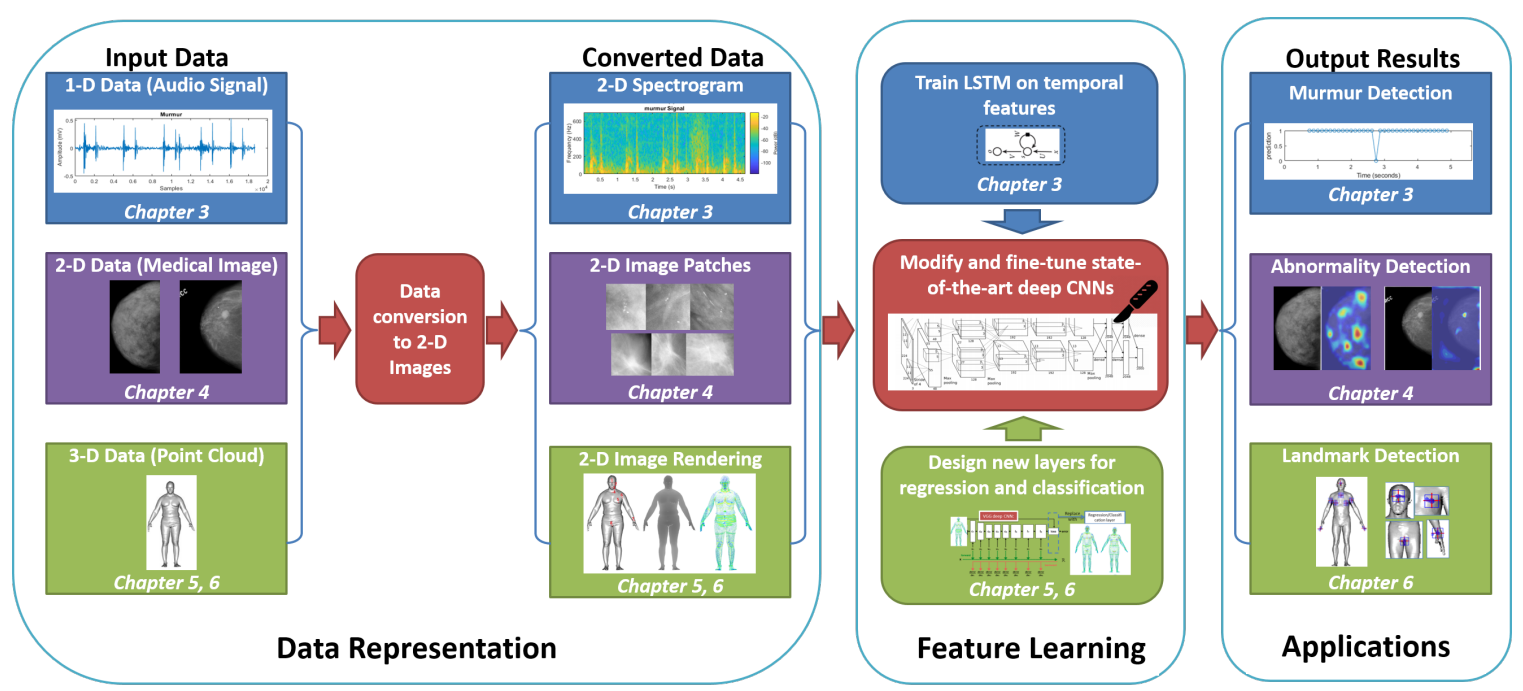

Figure 1: The proposed deep representation learning framework for 1D, 2D and 3D medical data analysis.

the effectiveness of transfer learning for the analysis of medical imaging data.

Studies also revealed that the brain's visual areas can be repurposed for other tasks, a phenomenon called visual cortex recruitment [11] [12]. In blind people, the visual cortex takes on higher cognitive functions, including language or speech processing. This discovery motivates us to convert other types of perception data, such as sound, into visual representations for conducting deep feature learning.

Note that while we draw an analogy between the human brain and our solution based on neural networks, we do not intend to create a computational model of the human brain. Instead, we adopt an engineering approach that mimics how brain works. In fact, the current research progress in neuroscience indicates that we know little about the human brain; therefore, our approach needs adapting to the progress in neuroscience. 


\subsection{Proposed Framework}

The diagram in Figure 1 illustrates the proposed deep representation learning framework for medical imaging data. The core of the framework comprises data conversions (for data representation) and architecture modifications (for feature learning). Both are highlighted with rounded rectangles in red. The proposed framework is built on deep CNN models that have been pre-trained on the ImageNet data set. When input data are in temporal sequences, the framework also integrates RNNs for achieving improved performance.

\subsubsection{Data Representation Module}

The data conversion component computes $2 \mathrm{D}$ visual representations for different types of medical imaging data. Data in different dimensions are unanimously converted to 2D representations in order to conduct transfer learning [10], which re-uses features learned from the ImageNet data set embedded with existing deep CNN architectures.

The data representation module converts medical imaging data to $2 \mathrm{D}$ visual representations with minimum information loss. In particular, 1D physiological signals are converted to 2D spectrograms. For 2D medical images, their resolution is usually too high for fine-tuning existing deep CNNs. Therefore, image patches are cropped to learn deep features for abnormality detection. 3D data, while perceived by human vision, become $2 \mathrm{D}$ visual perception in human brains. The framework uses an image rendering technique from computer graphics to convert 3D data to $2 \mathrm{D}$ representations for extracting deep features.

In short, the data representation module works on converting the raw data to new forms while highlighting information in the data for doing classifications or predictions. It is necessary that this step is revisited after training machine learning models in order to find the optimal data representation. 


\subsubsection{Feature Learning Module}

The architecture modification component makes operations (labeled with a scalpel

knife in Figure 1) on state-of-the-art deep CNN architectures. Architectural modifications are conducted to extend the capabilities of existing deep CNNs for solving real-life problems in medical data analysis.

We select deep CNN models and modify them for medical imaging data analysis based on transfer learning [10]. We choose the ones that lead to the best performance on the target applications. In these CNN models, features computed at the shallow end are general and hence transferable to a target task. Therefore we replace the layers towards the deep end with new ones for fine-tuning on the medical data. Depending on the application, the output layer is defined either for classification or regression. When the task is creating an abnormality map, special pooling layers are used. In the event that the input data contain temporal information, the modified CNN architectures are integrated with an RNN model. Along with the data representation module, the introduced modification and integration builds the framework for deep representation learning in spatial, spectral and temporal domains.

In short, the feature learning module leverages a plethora of deep architectures available for transfer learning. Experiments are conducted to decide which part of these models are to be kept and which new layers need to be added. Depending on the size of target data, a choice needs to be made on either fine-tuning the newly added layers or using the transferred architecture as off-the-shelf feature extractors.

\subsubsection{Applications to Medical Imaging Data Analysis}

We apply the proposed deep representation learning framework to selected applications in medical imaging data analysis. In the representation learning on 1D audio signal data, spatial features computed from deep CNNs are fed to a recurrent neural 
network (RNN) model named long-short-term-memory network (LSTM) to account for temporal features (in blue, Chapter 3). In the representation learning on 2D medical image data, deep CNNs are first trained on cropped image patches, and their final layers (fully connected and soft-max layers) are replaced and re-trained for computing a map of medical abnormalities (in purple, Chapter 4). In the representation learning on 3D human surface data, 2D image renderings are generated to compute viewpoint-invariant features for recognition (in green, Chapter 5), and to train new regression and classification networks for anatomical landmark detection (in green, Chapter 6).

\subsection{Contribution and Outline}

In this thesis, we propose a deep representation learning framework for medical imaging data analysis. The framework is designed for making effective use of deep learning for data-limited medical applications. It uses a unified data conversion module for data representation and modifies deep learning architectures for feature learning. The main contribution has been published in the following paper [13]:

- P. Xi, R. Goubran, C. Shu, "A Unified Deep Learning Framework for Multi-Modal MultiDimensional Data", IEEE International Symposium on Medical Measurements and Applications (IEEE MeMeA), Turkey, June 2019.

In Chapter 3, we apply the proposed framework on 1D medical data for cardiac murmur detection. We have converted phonocardiograms (PCG) to 2D spectrograms and trained deep CNNs for detecting cardiac murmurs. We have achieved a classification accuracy at $90.26 \%$. This has resulted in the following paper [14]:

- P. Xi, R. Goubran, C. Shu, "Cardiac Murmur Classification in Phonocardiograms using Deep Convolutional Neural Networks", International Conference on Pattern Recognition and Artificial Intelligence (ICPRAI), Montreal, Quebec, May 2018. 
In addition, in Chapter 3, we continue improving the above work by integrating the deep CNNs with an LSTM network to account for temporal features in the PCG sequence. We have achieved an improved classification accuracy of $94.01 \%$ and have published the results in the following book chapter [15]:

- P. Xi, R. Goubran, C. Shu, "Cardiac Murmur Classification in Phonocardiograms using Deep Recurrent-Convolutional Neural Networks", a book chapter in "Frontiers in Pattern Recognition and Artificial Intelligence" by World Scientific, https://doi.org/10.1142/11362, ISBN: 978-981-12-0335-0, July 2019.

In a different application, we have experimented on applying the framework to pilot workload prediction using electrocardiograms (ECG). The results have been published in the following paper [16]:

- P. Xi, A. Law, R. Goubran, C. Shu, "Pilot Workload Prediction from ECG Using Deep Convolutional Neural Networks", IEEE International Symposium on Medical Measurements and Applications (IEEE MeMeA), Turkey, June 2019.

In Chapter 4, we apply the proposed framework to 2D medical data for abnormality detection. We have extracted image patches from labeled abnormalities in mammogram images. Deep CNN models are trained on the cropped image patches rather than on the full-size mammograms, in order to learn features for detecting mass and calcification. Modifications are made to the deep patch CNNs for computing abnormality maps. This application has resulted in the following paper [17]:

- P. Xi, C. Shu, R. Goubran, "Abnormality Detection in Mammography using Deep Convolutional Neural Networks", IEEE International Symposium on Medical Measurements and Applications (IEEE MeMeA), Rome, Italy, June 2018.

In addition, in Chapter 4, we integrate the deep patch CNNs with region-proposal networks when the location of medical abnormalities are available during training. This integration has been validated on an ultrasound liver data set in the following journal paper [18]: 
- P. Xi, H. Guan, C. Shu, L. Borgeat, R. Goubran, "An integrated approach for medical abnormality detection using deep patch convolutional neural networks", Vis Comput (2019) doi:10.1007/s00371-019-01775-7.

Furthermore, we have conducted a comparative analysis on deep object detectors for the detection of medical abnormalities. The analysis results will be published in a book chapter [19]:

- P. Xi, G. Rouhafzay, H. Guan, C. Shu, L. Borgeat, R. Goubran, "Computer Aided Detection of Abnormality in Mammography using Deep Object Detectors", State-of-the-art in Neural Networks and Applications in Medical Field, by Elsevier, in press for 2020.

In another application, we have proposed an integrated approach using deep object detectors for detecting abnormalities in breast Magnetic Resonance Imaging (MRI) data. This work will be published as follows [20]:

- G. Rouhafzay, Y. Li, H. Guan, C. Shu, R. Goubran, P. Xi, "An Integrated Deep Approach for Lesion Detection in Breast MRI", ICPRAI 2020 - Second International Conference on Pattern Recognition and Artificial Intelligence, Zhongshan, China, 2020.

In Chapter 5, we apply the framework on 3D medical data for computing features robust to varying viewpoints. We have converted 3D human body surface data to 2D renderings with various viewing angles. Deep CNN models are then trained on the $2 \mathrm{D}$ renderings for recognition through learning viewpoint-independent features. This work has resulted in the following journal paper [21]:

- P. Xi, C. Shu, R. Goubran, "Comparing 2D Image Features on Viewpoint Independence Using 3D Anthropometric Dataset", Int. J. of the Digital Human, 1(4) (2016), pp. 412-425.

In Chapter 6, we continue applying the framework to 3D data for locating anatomical landmarks. We have converted 3D human body surface data to different 2D renderings. The landmark prediction is realized through a cascaded learning process. The locations of the selected landmarks are first estimated through a deep CNN, 
which is modified for doing regression. Their locations are further adjusted through a second deep CNN, which is modified for doing local classifications. This work has been published in the following paper [22]:

- P. Xi, C. Shu, R. Goubran, "Localizing 3D Anatomical Landmarks Using Deep Convolutional Neural Networks", 14th Conference on Computer and Robot Vision (CRV), Edmonton, Alberta, May 16-19, 2017.

The proposed framework leverages 2D deep features extracted through the deep CNNs for 1D, 2D and 3D medical data analysis. After dealing with the challenges on using deep learning for medical data analysis, the framework has been successfully applied to several medical data sets, all in limited quantities. It proves effective in learning deep representations from limited medical imaging data.

In summary, we have made the following contributions using the proposed framework for deep representation learning:

1. We proposed a deep representation learning framework and successfully validated it on medical imaging data. The framework is based on transfer learning since the target data are different from the source data. In the framework, medical data in different dimensions are unanimously converted to $2 \mathrm{D}$ visual representations for fine tuning pre-trained deep CNNs with adaptations. The framework is also extensible to more medical data analysis problems [13].

2. We proposed an algorithm to detect abnormalities in physiological signal data. It was based on visual representations of the spectrum of frequencies of signal data over time. With deep learning, we have found an optimal frequency range and the best spectral representations for cardiac murmur detection through computing spatial, spectral and temporal features [14-16] (Chapter 3);

3. We proposed an algorithm to detect medical abnormalities located in tiny local areas of high-resolution medical images. It is based on an integration of deep 
patch CNNs with class activation mappings and region-based CNNs. Through experiments, we found that existing deep CNNs had failed to detect abnormalities when trained on compressed full-view images. Instead, our framework used local views to conduct deep feature learning and the integrated deep detectors outperform traditional approaches by a large margin [17-19] (Chapter 4);

4. We proposed an algorithm to compute viewpoint-independent image features for human recognition. We demonstrated that applying deep learning to the computation of human shape features results in those being robust to varying viewpoints [21] (Chapter 5);

5. We proposed an algorithm to predict the location of 3D landmarks. We have discovered an optimal combination of visual representations for achieving the best performance. The task is framed as a combination of regressions and classifications in mimicking human vision mechanism [22] (Chapter 6).

The thesis is organized as follows. We conduct literature review on deep neural networks in Chapter 2. We then apply the proposed deep representation learning framework on medical imaging data in Chapters 3, 4, 5 and 6 respectively. Finally, we conclude the thesis in Chapter 7. 


\section{Chapter 2}

\section{Literature Review}

The proposed deep learning framework is based on convolutional neural networks $(\mathrm{CNN})$ and recurrent neural networks $(\mathrm{RNN})$. In this chapter, we first review the two main types of deep learning models, followed by related work on reviewing traditional features in computer vision and the history of deep CNNs.

\subsection{Machine Learning}

Real-life phenomena exist in various shapes and flavors. Traditional statistical approaches fit pre-defined models to data using linear, quadratic or logarithmic models; however, the data in modeling are usually complex, nonlinear or disorganized. Machine learning aims to discover patterns in the data without assuming distributions or relations between input and output variables.

In general, machine learning is categorized into the following three classes: supervised learning, unsupervised learning and reinforcement learning. Supervised learning is to develop predictive models based on both input and output data. It is used to solve classification or regression problems. Sample algorithms include support vector machines, naïve Bayes, nearest neighbor, discriminant analysis, neural networks, decision trees, ensemble methods, linear and non-linear regressions. 
Unsupervised learning is defined as grouping and interpreting data based on input data only. No labels are available for the input data. One big category of algorithms is clustering, which needs to figure out the classes based on grouping similar objects and creating rules on the similarities. Sample algorithms include k-means, fuzzy c-means, neural networks, Gaussian mixture and Hidden Markov Model (HMM) [23].

Reinforcement learning is an algorithm that knows a set amount of rules and makes moves so as to maximize some notion of cumulative reward. It differs from supervised learning in that labels need not to be presented, and sub-optimal actions need not to be explicitly corrected. Instead the focus is on performance, which involves finding a balance between exploration (of uncharted territory) and exploitation (of current knowledge). [24]

Theories, technologies and applications discussed in this thesis belong to supervised learning. From training data-set, features are manually or automatically extracted for training classifiers or regressors. The trained models are then tested on an independent data-set before they are deployed for applications. The implementations of different models are conducted using the latest version of Matlab (MathWorks, USA), unless indicated otherwise.

\subsubsection{From Feature Engineering to Architecture Engineering}

A machine learning system is mainly composed of feature extraction and classifier building. While traditional machine learning conducts these steps separately, latest deep learning technologies provide an end-to-end solution by combining feature extraction and classification in a single architecture.

Fundamental to the application of machine learning is feature extraction, which is both difficult and expensive [25]. It can be categorized as traditional feature engineering and the most recent deep learning approaches (also deemed as architecture 


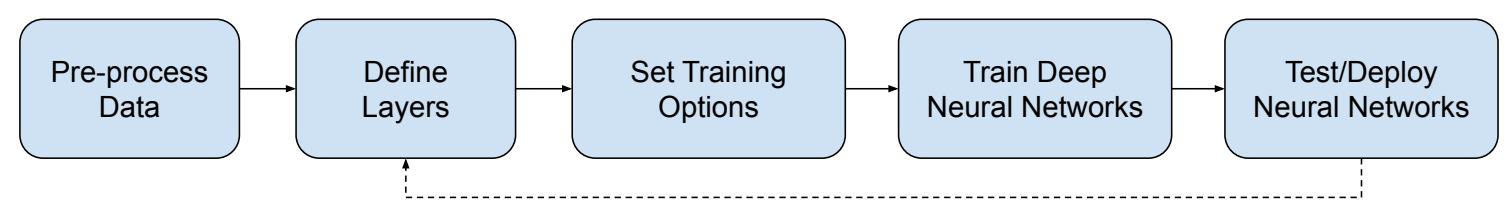

Figure 2: Work flow of deep learning applications.

engineering). The former is to use domain knowledge of data to manually create features that work well with machine learning algorithms (or models). In comparison, the deep learning approaches learn features from data based on the tasks they are trained for. Although deep learning computes features automatically, the design and creation of novel deep neural network architectures require expertise, knowledge and creativity on the selection of building blocks and the design of structures and layouts.

\subsubsection{Deep Learning}

A typical deep learning work-flow is illustrated with Figure 2. Building a deep learning model includes defining the layers and setting options for training. Depending on the actual performance of the trained networks and application requirements, some of the steps are repeated to improve the performance (dotted line).

Depending on data types, most deep learning neural networks can be categorized into two types: deep CNNs and deep RNNs. The former works well on spatial data such as images or those that can be converted to image representations, and the latter works on temporal data such as speech signals or natural language.

\section{Deep Convolutional Neural Networks}

Typical deep CNNs comprise three parts: convolution layers for searching for patterns in the input (use small filters to search for patterns in the input images), max pooling for down-sampling (reduce dimensionality while preserving important information), 
and ReLU (rectified linear units layer, typically converts negative numbers to zero) for introducing nonlinearity. CNNs typically end with three layers: fully connected layer, softmax layer and classification layer. Fully connected layers look at which high-level features correspond to a specific class and calculates scores for each class. Softmax layer turns scores into probabilities. Classification layer categorizes inputs into one of the classes.

With a large amount of training data and good knowledge about architecture design, deep CNNs can be built and trained from scratch. Otherwise a good practice is to use transfer learning with pre-trained neural networks [10]. Since the pre-trained models are great feature extractors, it is a good starting point to perform deep learning by fine-tuning parameters in pre-trained deep neural networks. For example in image classifications, initial layers learn low level features such as edges or corners and deeper layers learn high level features like objects. In order to learn features specific to a new dataset, the final layers need to be replaced with new convolutional and/or fully connected layers and get fine-tuned.

\section{Deep Recurrent Neural Networks}

An RNN is a class of artificial neural networks where connections between nodes form a directed graph along a sequence in order to encode and decode temporal dynamic behavior in a time sequence [26]. The architectures of RNNs come in many variants, among which the most notable are long short-term memory (LSTM) and gated recurrent unit (GRU).

The LSTM is a deep learning system augmented by recurrent gates called "forget" gates, which allow errors to flow backwards through unlimited number of virtual layers unfolded in space. Hence, LSTM enables learning from memories of events that happened millions of discrete time steps earlier. In comparison, GRU has fewer parameters than LSTM but has similar performance in applications such as music 
modeling and speech signal processing [27].

A variant of RNN called bi-directional RNNs uses a finite sequence to predict each element of the sequence based on the past and future contexts [26]. It is achieved through concatenating the outputs of two RNNs, one processing the sequence from left to right and the other from the opposite direction. This technique proves to be useful when combined with LSTM $[28,29]$.

\section{Transfer Learning}

Transfer learning is to fully or partially reuse existing deep learning architectures and their connection weights for domain-specific tasks. These pre-trained deep models can be used just for feature extraction or fine-tuned on domain-specific data set for the best performance.

In general there are two ways of conducting transfer learning with deep CNNs. A deep CNN that has been trained on natural images such as ImageNet has the features computed at different layers ranging from being generic to specific. Given a new target data set, the first part of the pre-trained deep CNN, including its weights, can be reused as a feature extractor, of which the output are then fed to a traditional classifier (e.g., support vector machine) for training. Another way is to append new output layers to the feature extractor and fine-tune the new model.

In a discussion on how transferable features are in deep neural networks [10], the authors claimed that first-layer features are not specific to a particular dataset or task, but general in that they are applicable to many data sets and tasks. It was mentioned that the transferability of deep features is negatively affected by the specialization of higher layer neurons to their original task. It was concluded that the transferability of features decreases as the distance between the base task and target task increases, but that transferring features even from distance tasks can be better than using random features. 


\subsection{Related Work}

In visual computing, tremendous progress has been made by deep CNNs in object classification and recognition, thanks to the availability of large scale annotated datasets such as ImageNet Large Scale Visual Recognition Competition (ILSVRC) [2]. Comparing to hand-crafted image features, deep CNNs automatically extract features from a large data-set for tasks they are trained for.

\subsubsection{Feature Engineering}

\section{One-dimension Signal Data}

For one-dimensional signal data, the following features are normally extracted for classification purposes: simple statistical features, time domain and frequency domain features. Example statistical features include means, standard deviations, skewness, Kurtosis, maximum and minimum values; example time domain features include Cross-correlations between each dimension, auto-correlations, orders of the autoregressive (AR), and integrated (I) and moving average (MA) part of an estimated ARIMA model; frequency domain features include frequencies of the $k$ peaks in amplitude in the DFTs for the detrended $d$ dimensions and $k$-quantiles of the DFTs.

\section{Two-dimension Image Data}

Good image features should have both geometric invariance (translation, rotation, scale) and photometric invariance (brightness, exposure). The following are wellknown 2D image features: scale invariant feature transform (SIFT), Local Binary Patterns (LBP), histogram of oriented gradients (HOG), KAZE features, speed-up robust feature (SURF) and binary robust invariant scalable key points (BRISK).

The LBP feature was proposed in 1990 as a powerful feature for texture classification $[30,31]$. To compute LBP feature, the image is first divided into smaller cells. 
Within each cell, a histogram is computed on the frequency over the number of center pixels which have greater pixel values than neighbors and the number of those with a lower pixel value. Normalizing and concatenating the histograms forms a feature vector for the image.

The HOG feature was introduced in 2005 for the purpose of object detection [32]. The first step is to compute gradient values along the horizontal and vertical directions on the image. The second step is to create the orientation-based cell histograms by counting a weighted vote from the cells. To account for changes in illumination and contrast, the cells need to be grouped together into spatially connected blocks. The HOG descriptor is a concatenated vector of the normalized cell histograms from all the blocks.

\section{Three-dimension Geometry Data}

A $3 \mathrm{D}$ shape signature is referred to as a local description for a point on a 3D surface and shape descriptor is referred to as a global description for the entire shape. The following are popular 3D features: global point signature (GPS), heat kernel signature (HKS), wave kernel signature (WKS), temperature distribution descriptor (TD) and unique signatures of histograms for surface and texture description (SHOT).

\subsubsection{Deep CNN models}

Neural networks are inspired by the nervous system where the nodes correspond to neurons and the edges to synapse. Depending on applications, the structure of the network can be designed to learn weights in the hidden layers by minimizing a cost function at the output layer. Multi-layer neural networks serve as a good feature learning tool as they learn the representations at the hidden layers for classification or regression. 
Deep neural networks is structured hierarchically by stacking multiple layers of simple learning blocks such as Restricted Boltzmann Machine (RBM) [33] and Autoencoder [34]. The output of each hidden layer is considered as a representation of the input data. Using this representation as input, the following layer produces new representations at output, which is then fed into a higher-level layer. The output of the last layer servers as a lower-dimensional feature or representation [35]. The deep convolutional neural network has demonstrated tremendous improvement on many image and vision tasks [7,36-38].

The advantage of deep neural networks is that it is an end-to-end system, alleviating the need for manually designing a feature extractor; however, deep neural networks have a ravenous appetite for labeled training data. Transfer learning is a good solution to the problem of insufficient training data when using deep neural networks. It has proved an effective approach by taking advantage of the deep neural networks as a feature extractor [5,39,40]. Razavian et al. [41] discussed that the generic descriptors extracted from deep CNNs are very powerful. They used features extracted from the OverFeat network [42] as a generic image representation to tackle a range of image tasks. To use transfer learning, trained models/networks are available from repositories including the model zoo of Caffe project [43].

\section{AlexNet}

In 2012, Krizhevsky et al. [6] entered ILSVRC with a deep CNN (named AlexNet) and achieved top- 5 test error rate of $15.3 \%$, compared to $26.2 \%$ achieved by the second-best entry. AlexNet contains eight learned layers - five convolutional and three fully-connected.

The network has 60 million parameters and 650,000 neurons, consisting of five convolutional layers, some of which are followed by max-pooling layers, and three fully-connected layers with a final 1000-way softmax layer. They use non-saturating 
neurons to make training faster and use a regularization method called "dropout" to reduce over-fitting. It was found that removing any convolutional layer resulted in inferior performance (loss of about $2 \%$ for the top-1 performance).

In general, each of the convolution layers contains no more than 1 percent of the model's parameters and most of the parameters reside in the fully-connected layers. The first convolutional layer has learned a variety of frequency- and orientationselective kernels as well as various color blobs.

\section{VGGNet}

In 2014, Simonyan and Zisserman [7] introduced a deeper 19-layer CNN (named VGGNet) and achieved top result in the localization task of ImageNet ILSVRC. The network used very small $3 \times 3$ convolutional filters and showed significant improvement. This influential work indicated that CNNs need to have a deep network of layers in order for the hierarchical feature representations to work.

In the paper of AlexNet, the authors emphasized that the layers are essential in that removing any of the layers will lead to a drop in performance. The paper of VGGNet actually studies on increasing the number of layers by making it deeper. More specifically, they fix other parameters of the architecture, and steadily increase the depth of the network by adding more convolutional layers, which is feasible due to the use of very small $(3 \times 3)$ convolution filters in all layers.

This work investigates the effect of the convolution network depth on its accuracy in the large-scale image recognition setting. Their work secured the first and the second places in the localization and classification tracks in ImageNet Challenge 2014. They also show that the representations generalize well to other datasets.

The main contribution is a thorough evaluation of networks of increasing depth using an architecture with very small $(3 \times 3)$ convolution filters, which shows a significant improvement on the prior-art by pushing the depth to 16-19 weight layers. 


\section{GoogLeNet}

The previous CNNs have a typical structure - stacked convolutional layers followed by optional normalization and pooling layers. Lin et al. [44] proposed an approach called network in network to increase the representational power of neural networks. They added multi-layer perceptron after convolution layers (called mlpconv) to make the network more powerful.

In 2014, Szegedy et al. [8] introduced a deeper CNN (named GoogLeNet) to ILSVRC and achieved top 5 error rate of $6.7 \%$. Instead of sequentially stacking layers, this network was one of the first CNNs that used parallel structures in its architecture (9 Inception modules with over 100 layers in total). Their architecture is called Inception (incarnated as GoogLeNet in the challenge).

GoogLeNet is developed based on LeNet architecture by adding inception module. The main idea of the Inception architecture is based on finding out how an optimal local sparse structure in a convolutional vision network can be approximated by readily available dense components. The architecture needs to find the optimal local construction and to repeat it spatially. As a result, the architecture uses a combination of $1 \times 1,3 \times 3$ and $5 \times 5$ convolutions with their output filter banks concatenated into a single output vector forming the input of the next stage. Adding a parallel pooling path also has additional benefit.

\section{ResNet}

Deeper neural networks are more difficult to train. In [9], The authors present a residual learning scheme (named ResNet) to facilitate the training of neural networks much deeper than previous ones. It is known that simply adding more layers to a deep model leads to higher training error. The authors make a hypothesis that it is easier to optimize the residual mapping than to optimize the original, unreferenced 
mapping. To achieve this, they use a building block for residual learning.

Denoting the desired mapping as $\mathcal{H}(x)$, this building block breaks the mapping into stacked nonlinear layers $\mathcal{F}(x)$ and an identity mapping. Therefore, the original mapping is recast into $\mathcal{F}(x)+x$. In their implementation, the weight layers are replaced with small convolution layers such as $1 \times 1$ or $3 \times 3$, following the similar practices found in the design of previous deep neural networks. There are two different versions of the building block using different weight layers [9].

The building block seems a trivial change; however, this change leads to great benefits in building much deeper neural networks. As the paper claims, the extremely deep residual nets by stacking multiple building blocks are easy to optimize and enjoy accuracy gains from greatly increased depth, producing results substantially better than previous networks. In ILSVRC 2015, The new 152-layer network architecture set new records in achieving 3.57\% error rate in the classification task. The residual learning framework is 8 times deeper than VGGNet but still has lower complexity.

In the order of being introduced, the above neural network architectures become deeper and more complex. The first efforts were to make them deeper and then new modules and parallelism were introduced to make them more powerful in modeling the data. Lastly, in order to avoid over-fitting, small convolutional filters are commonly used in all the architectures. Along this path, a few new deep CNN models have been introduced, including Xception [45], Inception-v4 [46], and ResNeXt-50 [47]. This is by no means a complete list of all the neural networks invented so far but rather serves as a glimpse into the evolving path of deep convolutional neural network architectures. 


\section{Chapter 3}

\section{Representation Learning on 1D Physiological Signals}

We introduce the deep representation learning framework for predicting cardiac murmurs from phonocardiograms. A successful validation on this type of data is important for many applications, including physiological monitoring, speech recognition and digital signal analysis. In the proposed deep learning framework, components used for this validation are highlighted in Figure 3.

Cardiac murmurs are the first signs of pathological changes in heart valves. Their subtle presence poses great challenges for detection through auscultation or phonocardiograms (PCGs). Therefore, computer-aided detection (CADe) of heart murmurs has medical significance in assisting health care professionals. Traditional CADe approaches relying on engineered features are prone to changes in environmental noise and data collection methods, whereas deep Convolutional Neural Networks (CNN) have shown robustness in advancing the performance of computer vision tasks through automatic feature learning from large amount of data. Meanwhile, deep Recurrent Neural Networks (RNN) have demonstrated state-of-the-art performance on processing sequence data such as in speech recognition and natural language processing. With 


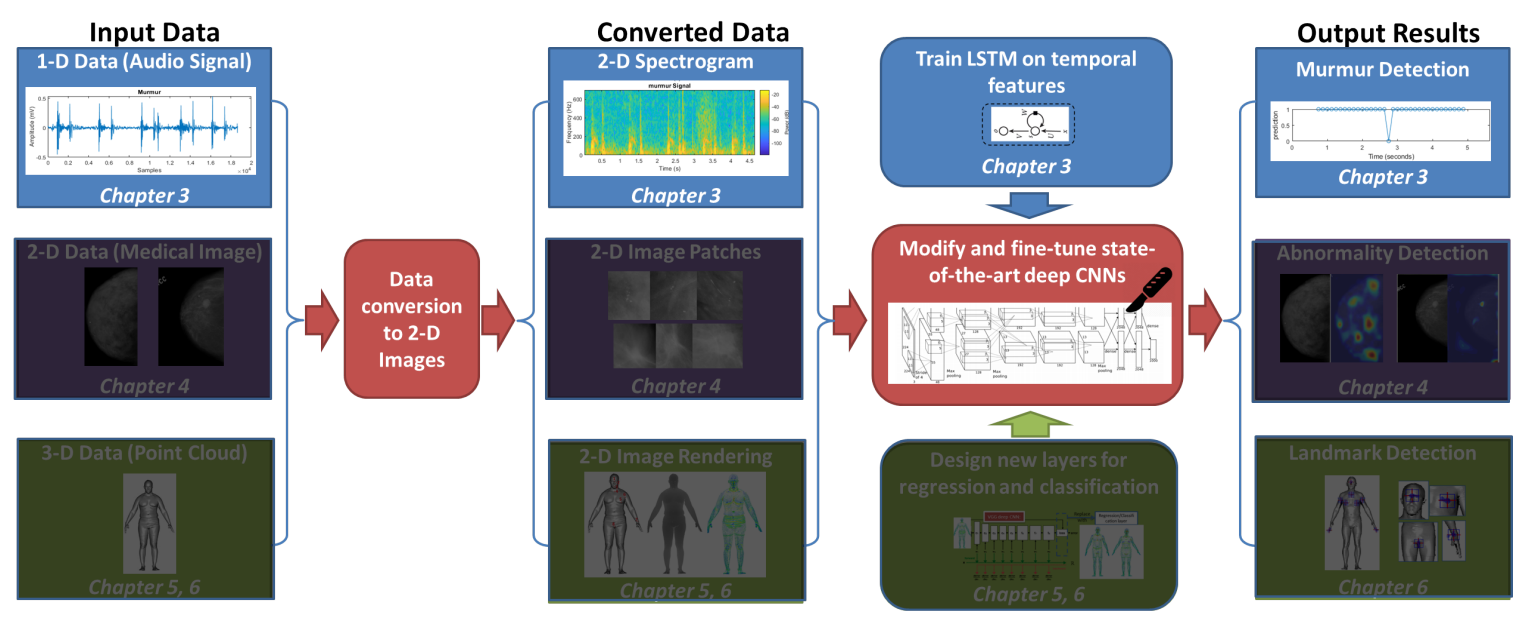

Figure 3: Applying the deep representation learning framework on 1D audio signal data (from left to right: raw data, converted 2D data representations, deep model training and output).

a limited set of labelled PCG recordings, this work first transforms PCGs to topologypreserving spectral images, conducts data augmentation and successfully tunes latest deep CNN architectures with transfer learning. Experimental results indicate that the fine-tuned deep CNNs are of effectiveness in classifying cardiac murmurs from PCG recordings without segmentation. Moreover, the deep CNNs gained further performance boost from learning the dynamics in temporal sequences after being plugged into an RNN model. In summary, the proposed deep recurrent-convolutional neural network approach captures spatial, spectral and temporal features. It achieves an F-score at 0.9767 for automatic cardiac murmur detections in phonocardiograms.

\subsection{Introduction}

According to World Health Organization (WHO), cardiovascular disease (CVD) is the leading cause of mortality worldwide [48]. The majority of the problems found in CVD 
are related to heart valves, in which cardiac murmurs are the first signs of pathological changes. Therefore, classic auscultation and phonocardiography (PCG) play essential roles in early detection of CVDs, providing guide for further examinations.

Cardiac valve problems include mitral valve prolapse, mitral valve or aortic stenosis, aortic sclerosis and stenosis, and mitral or aortic regurgitation, etc. Stenosis leads to heart working harder to pump blood to the rest of the human body. Regurgitation means the blood is going the wrong way through the valves, resulting in heart working harder to force blood through the damaged valves. Both can wear out the heart and lead to heart failure.

Cardiac murmur is the sound of blood flowing through a problematic heart valve. It may also be a condition which makes the heart beat faster and forces heart to handle more blood quicker than normal. Most heart murmurs are innocent and do not require treatment; however, murmurs linked to damaged or overworked heart valves need surgery or treatment. Some murmurs are congenital and others are a part of ageing or from other heart problems.

The evaluation of cardiac murmurs is based on its timing, shape, location, intensity and duration in the cardiac cycle (S1, systolic, S2 and diastolic). They can be categorized into systolic or diastolic murmurs. In each situation, the murmur takes a particular shape, e.g., crescendo-decrescendo shape for aortic stenosis, decrescendo shape for aortic regurgitation, plat shape for mitral regurgitation or tricuspid regurgitation. This implies that visual representations of heart recordings (in time and frequency domains) can be utilized for detecting cardiac murmurs.

Because of the medical significance of cardiac murmurs, there has been considerable effort on developing computer-aided detection (CADe) approaches [49-51]. Conventional approaches follow three steps: segmentation, feature extraction and classification. The features used in CADe are selected in three domains: time, frequency, and time-frequency. While the manual features work in certain cases, they 
are prone to changes in environmental noise and data recording instruments.

Recent advances in deep neural networks have enabled automatic feature learning from large amount of training data, providing an end-to-end solution from feature extraction to classifier building. Moreover the learning scheme contributes to the robustness of deep neural networks to dataset noise, making them suitable for solving the cardiac murmur classification problem.

Among deep neural networks, Convolutional Neural Networks (CNN) are especially effective in processing visual data. Therefore, deep CNNs are ideal candidates for cardiac murmur classifications because of the following reasons. First, with minimum information loss, PCGs can be transformed to spectrograms, a visual form that can then be processed through deep CNNs for extracting spatial and spectral features. Second, deep CNNs learn hierarchical features automatically from data. Therefore, they provide a powerhouse for feature learning in order to distinguish cardiac murmur recordings from normal ones.

Likewise, Recurrent Neural Networks (RNN) have demonstrated state-of-the-art performance on applications such as speech recognition and natural language processing. Therefore, RNNs are a natural fit for analysing PCGs or the transformed spectrogram "movies" in order to capture the dynamics in temporal sequences for detecting cardiac murmurs.

This chapter addresses the following questions: i) how deep CNNs will learn from a limited amount of labelled PCG recordings, ii) whether deep CNNs are robust in classifying PCGs without segmentation, iii) whether the fine-tuned models gain the same performance boost as they achieved in ImageNet Large-Scale Visual Recognition Challenge (ILSVRC) [2], and iv) whether the incorporation of temporal features helps improving the performance on cardiac murmur classification.

To address the limited training data problem, we employ data augmentation and transfer learning to fine-tune state-of-the-art deep CNN architectures. To address 
the other questions, we compare two approaches: i) we train and test deep CNNs on sampled spectrogram patches, and ii) after applying the deep CNNs for feature extraction, we train a Long Short-Term Memory neural network (LSTM) to account for temporal features in the sequence data.

Experimental results show that the best performing model in the first approach reaches an overall accuracy at $90.26 \%$, which is further pushed to $94.01 \%$ after the inclusion of temporal features. After experimenting on different spectral representations, an F-score of 0.9767 is achieved by mel spectrograms. This indicates that the proposed approach is promising for solving the problem of classifying cardiac murmurs in phonocardiograms automatically.

In summary, our approach enables the extraction of features for identifying the subtle and challenging differences between cardiac murmur and normal PCGs without segmentation. Our contributions are three-fold:

- Leveraged deep CNNs' hierarchical feature extraction capabilities through transfer learning.

- Tuned deep CNNs on a small training dataset without over fitting through data augmentation.

- Captured spectral, temporal and spatial features in PCG recordings for detecting cardiac murmurs through the integration of deep CNNs with RNNs.

\subsection{Related Work}

In this section, we first review the top three results on classifying normal and abnormal heart sound recordings in the PhysioNet/CinC challenge 2016 [52]. What is common among the winning methods is the use of shallow neural networks. In contrast, our work attempts to use deep neural networks because of their demonstrated 
advantage over traditional methods in many computer vision tasks. However, one of the challenges in training deep neural networks with limited data is to avoid over fitting. Therefore, we discuss the many techniques for dealing with the problem. In addition, we discuss recent work on applying deep recurrent and convolutional neural networks on sequence data.

Potes et al. [53] proposed an approach of using ensemble of feature-based and deep learning-based classifiers for the detection of abnormal heart sounds. The classifier ensemble approach obtained the highest score in the competition. Their classifier trained CNNs using PCGs decomposed into four frequency bands. Each of the CNNs consists of three layers: the input layer followed by two convolution layers.

Zabihi et al. [54] used ensemble of neural networks without segmentation and achieved the second best score in the PhysioNet/CinC Challenge 2016. They used 20 feed-forward neural networks - two hidden layers in each network and 25 hidden neurons in each layer. A combination rule is then applied to identify the quality and abnormality of each input.

Kay and Agarwal [55] built a fully-connected two-hidden-layer neural network trained by error back-propagation, and regularized with DropConnect to classify heart sounds as normal or abnormal.

The above approaches all use shallow neural networks as part of their solution mainly due to the limited amount of training data. Other factors that may affect their performance include the manually decided thresholds used in the combination rules [54] and the decomposition of frequency bands [53].

Limited amount of training data leads to overfitting in deep neural networks. Solutions include dropout, batch normalization and transfer learning [56]. Among all, the simplest is to add regularization term on the connection weights. Dropout works by removing neurons or connections from the network probabilistically during training [57]. Batch normalization works by normalizing layer inputs. It allows the use 
of much higher learning rates and results in less sensitivity to weight initialization [58]. In addition, Yosinski et al. [10] proposed a transfer learning approach that makes use of a neural network which has been pre-trained on large amount of data and finetune some of the layers . Other transfer learning approaches include learning from unlabeled data with stacked auto-encoders and then transfering the knowledge to a labeled dataset [59].

Data augmentation is another way to avoid over fitting in deep learning. It works by increasing the amount of training data from the existing training set. In image processing, this includes operations such as cropping, flipping and rotating the original images. It has been proved effective in avoiding over fitting.

Because of the success from recurrent neural networks on sequence data, there has been recent work on using deep CNNs and RNNs to detect features in electroencephalograms (EEG) [60,61]. Bashivan et al. [61] demonstrated a technique on learning representations from EEG data that are invariant to inter- and intra-subject differences in the context of mental load classification task. They transformed EEG activities into a sequence of topology-preserving multi-spectral images and trained a deep recurrent-convolutional network to learn representations from the sequence of images. While VGGNet was used in their work, a recent study by Xi et al. [14] indicates that AlexNet shows better performance in processing PCG data.

Another recent work on using deep CNNs and RNNs comes from Thodoroff et al. [60]. They followed a similar approach on learning spatial, spectral, and temporal structure of EEG signals for automatic seizure detection. Transfer learning was used to deal with limited data problem and for cross-patient detection of seizures. 


\subsection{Methodology using Deep CNNs}

\subsubsection{Data Selection}

To study heart sound problems, there are four publicly available heart sound databases: (i) the PhysioNet/CinC Challenge 2016 [52], (ii) the PASCAL Heart Sounds Challenge 2011 [62], (iii) the Michigan heart sound and murmur database (MHSDB) and (iv) the Cardiac Auscultation of Heart Murmurs database (eGeneralMedical). The PhysioNet/CinC dataset is by far the largest dataset with heart sound recordings labelled as normal and abnormal; however, no further labels on murmurs are available. The MHSDB and eGeneralMedical datasets have very limited data. Ultimately the PASCAL dataset is selected for this work as it comprises at-scale heart sound recordings labelled as normal or murmur.

From PASCAL dataset, we selected a subset which was collected from a clinical trial in hospitals using a digital stethoscope. Within the dataset, 66 murmur recordings and 200 normal recordings are used for this study. All the recordings were sampled to 4,000 $\mathrm{Hz}$ and their length varies from 1 second to 30 seconds.

\subsubsection{Data Pre-processing}

To take advantage of the automatic feature extraction capabilities of deep CNNs, PCGs are first transformed to spectrograms. According to Ranganathan et al. [63], most cardiovascular murmurs show a frequency range extending from almost zero to $700 \mathrm{~Hz}$. To compute spectrograms, we applied a 512-point Hamming window with 75\% overlapping for computing Fast Fourier Transform (FFT) of size 512. A plot of the spectrograms computed on one murmur PCG and one normal PCG is shown in Fig. 4, in which limits of the color axis for spectrograms are set between -120db and $-40 \mathrm{db}$. 

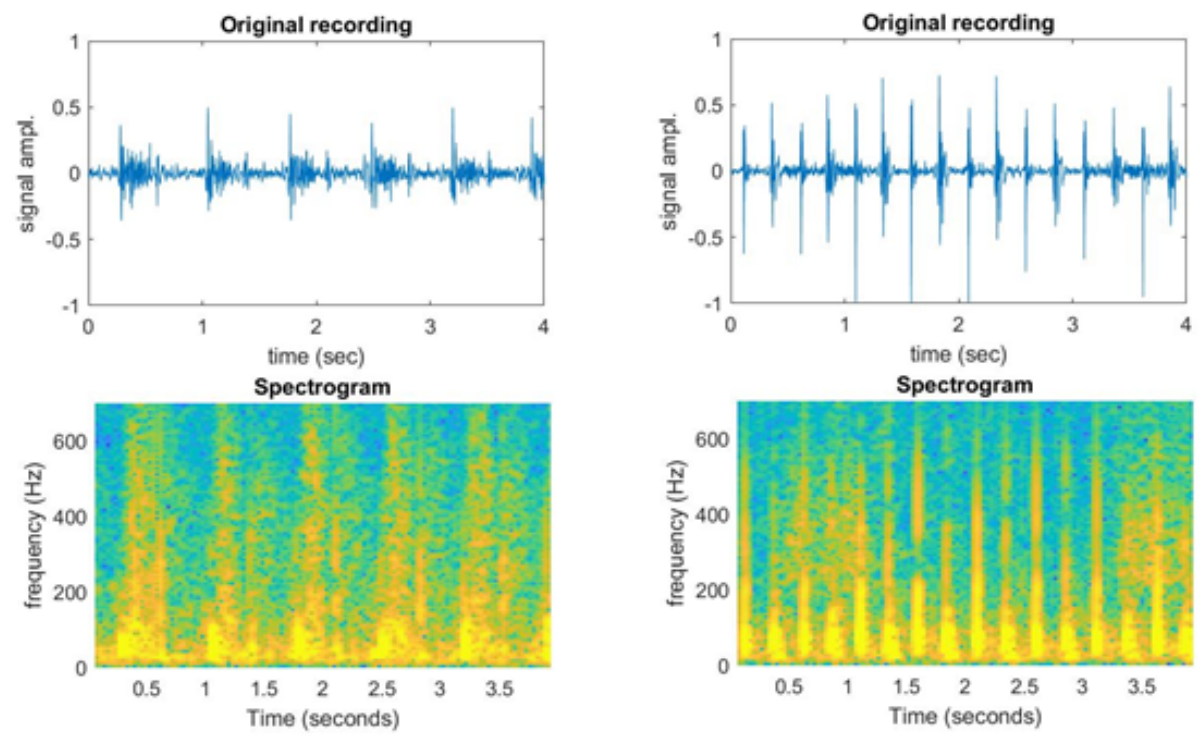

(a)

(b)

Figure 4: PCG recordings and spectrograms computed for (a) a murmur subject and (b) a normal subject.

Our motivation is to conduct feature extraction and classification from data automatically. Traditional approaches segment PCGs into S1, systole, S2 and diastole, and train classifiers on manually designed features. Instead, this work performs automatic feature extraction and conducts classifications without the need of segmentation.

\subsubsection{Data Augmentation}

An observation on the PCGs and computed spectrograms shows that most cardiac cycles last no more than 1.5 seconds. Therefore, to prepare training images for deep CNNs, a sliding window with length of 1.5 seconds is used to extract image patches from spectrograms with an initial step size of 500 samples. As a result, those heart recordings with a length shorter than 1.5 seconds are excluded from this study, leaving the final number of training subjects for murmur and normal categories to be 64 and 180 respectively. 
All the windowed image patches are put into two categories, i.e., murmur or normal, and a pre-trained deep CNN is modified to classify two classes at the output. Sample image patches from the two categories are plotted with Fig. 5.

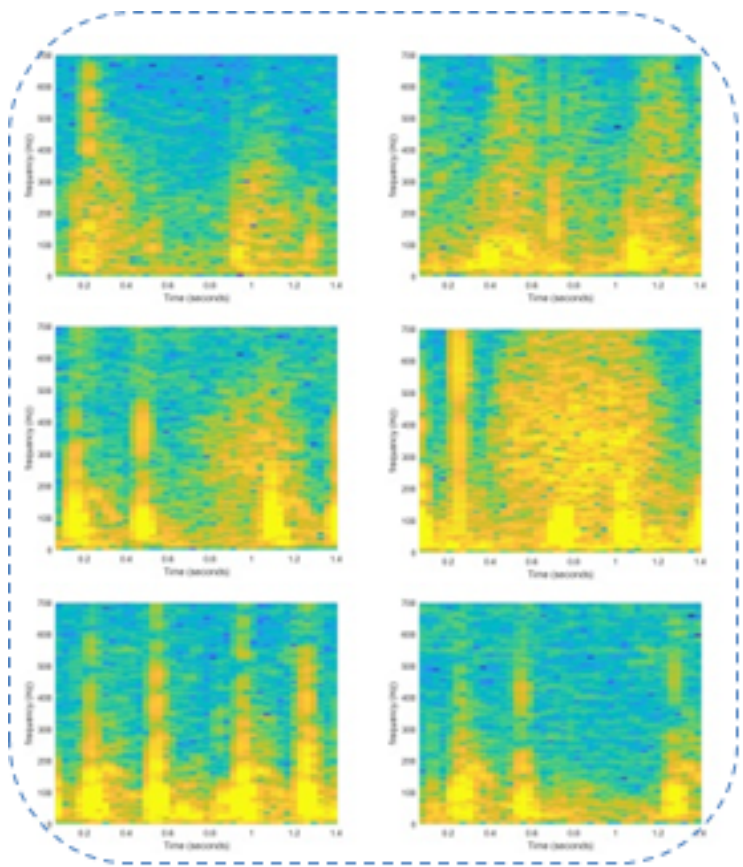

(a)

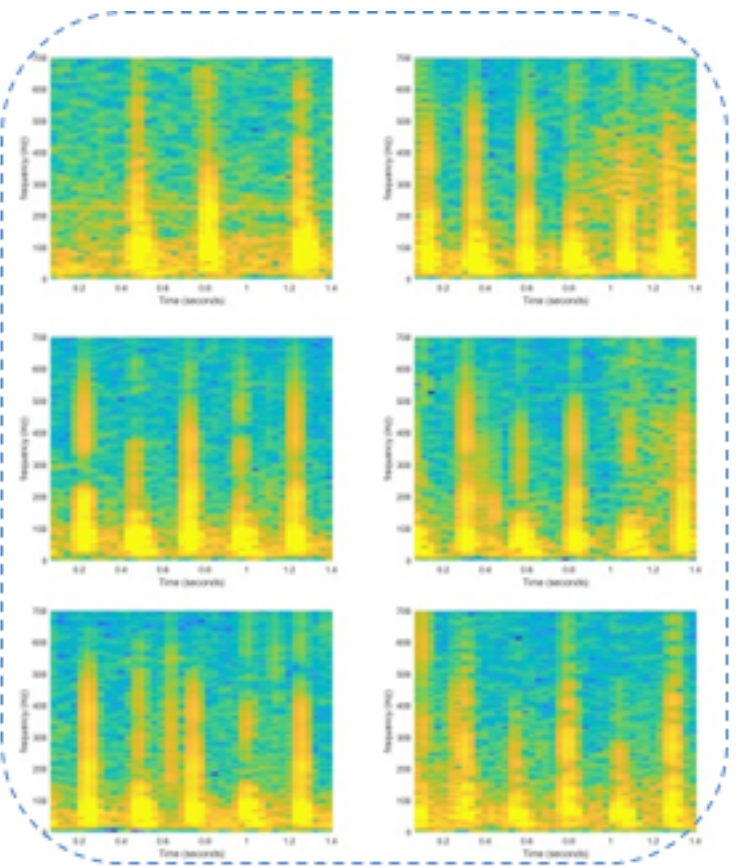

(b)

Figure 5: Sample image patches extracted from spectrograms within (a) murmur and (b) normal categories.

We conduct five-fold cross validations on the training dataset. For each fold, an independent testing set of 12 subjects are set aside for the murmur category and a testing set of 35 subjects are for the normal category. The remaining subjects within each category are used to extract image patches for training purpose. The grouped image patches are used to fine-tune deep CNNs.

\subsubsection{Feature Extraction and Model Building}

Following one of the transfer learning approaches, we feed the image patches from both categories into the modified deep CNNs for fine-tuning. The first part of each 
model is frozen, leaving the last layers actively tuned for high performance.

To test trained deep CNN models, we apply the same sliding window approach (same width and step size as those used for extracting training image patches) to consecutively extract image patches along a spectrogram. Each patch is fed into the trained deep CNN to predict a label. The predicted labels are then agglomerated to a voting scheme for creating a final prediction. The approach is illustrated in Fig 6 .

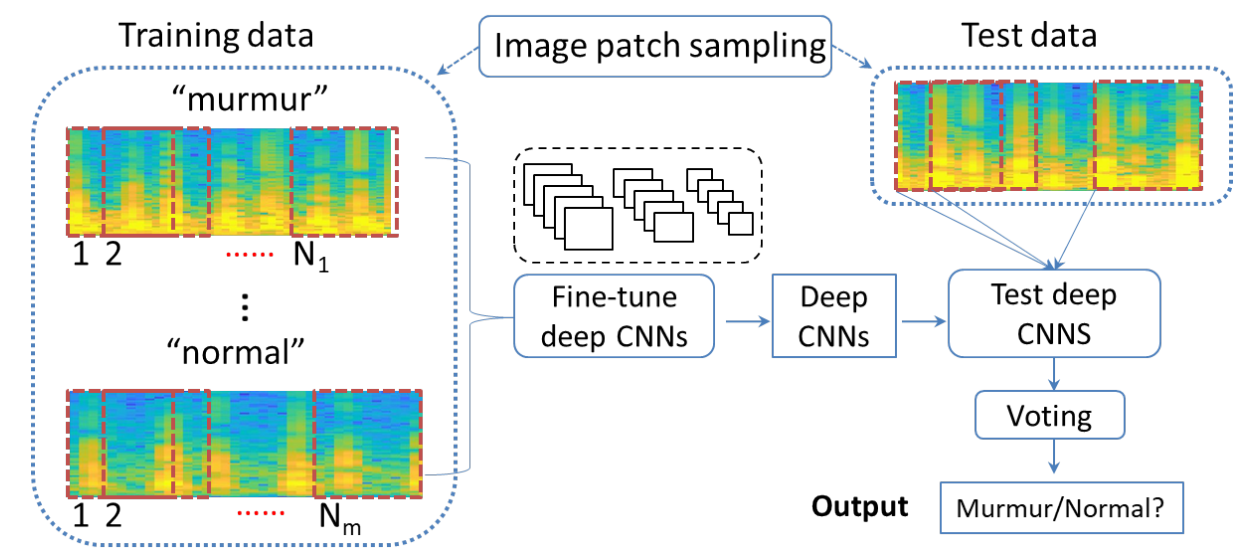

Figure 6: Diagram of a murmur detection approach using deep CNNs.

\subsection{Methodology using Deep Recurrent CNNs}

Deep convolutional neural networks have demonstrated good performance on extracting spectral and spatial features automatically from visual data. Essentially PCG signals are sequence data and therefore temporal features need to be learned for combining with the visual features for detecting cardiac murmurs. Because of the recent successes on applying recurrent-convolutional neural networks on EEG signals [60,61], we propose an approach on combining the deep convolutional neural networks with recurrent neural networks for the detection of cardiac murmurs in PCG signals.

To learn temporal features, we select long short-term memory (LSTM) network [64], a type of RNN for studying sequence and time-series data. An LSTM network 
uses memory cells and gated inputs/outputs to learn long-term dependencies between time steps of a sequence. The LSTM networks can study the sequence in forward directions or in both forward and backward directions (bi-directional).

\subsubsection{Data Selection}

We use the same dataset as those used for training deep CNNs. To recall, the number of sequences for murmur and normal classes are 66 and 200, respectively.

\subsubsection{Feature Extraction and Model Building}

The approach is illustrated in Figure 7. PCG signals are first transformed to spectrograms using Fast Fourier Transform (FFT), from which a sliding window is used to sample image patches (step size $=100$ samples). Selecting one of the fine-tuned deep CNNs from Section 3.3, we can compute for each image patch a feature vector, which captures spatial and spectral patterns. After feeding the feature vectors into a bi-directional LSTM network, a classifier is trained to learn the temporal pattern. As a result, a deep recurrent-convolutional neural network is built for cardiac murmur detection.

The following settings are used to design the architecture of the bidirectional LSTM neural network. The input sequence layer size is set as the length of the feature vector computed from the deep CNN. A bidirectional LSTM layer is added with an output size of 100. The output of the LSTM is defined at the last unit, followed by a fully connected layer of size two (a two-class classifier), a softmax layer and a classification layer. 


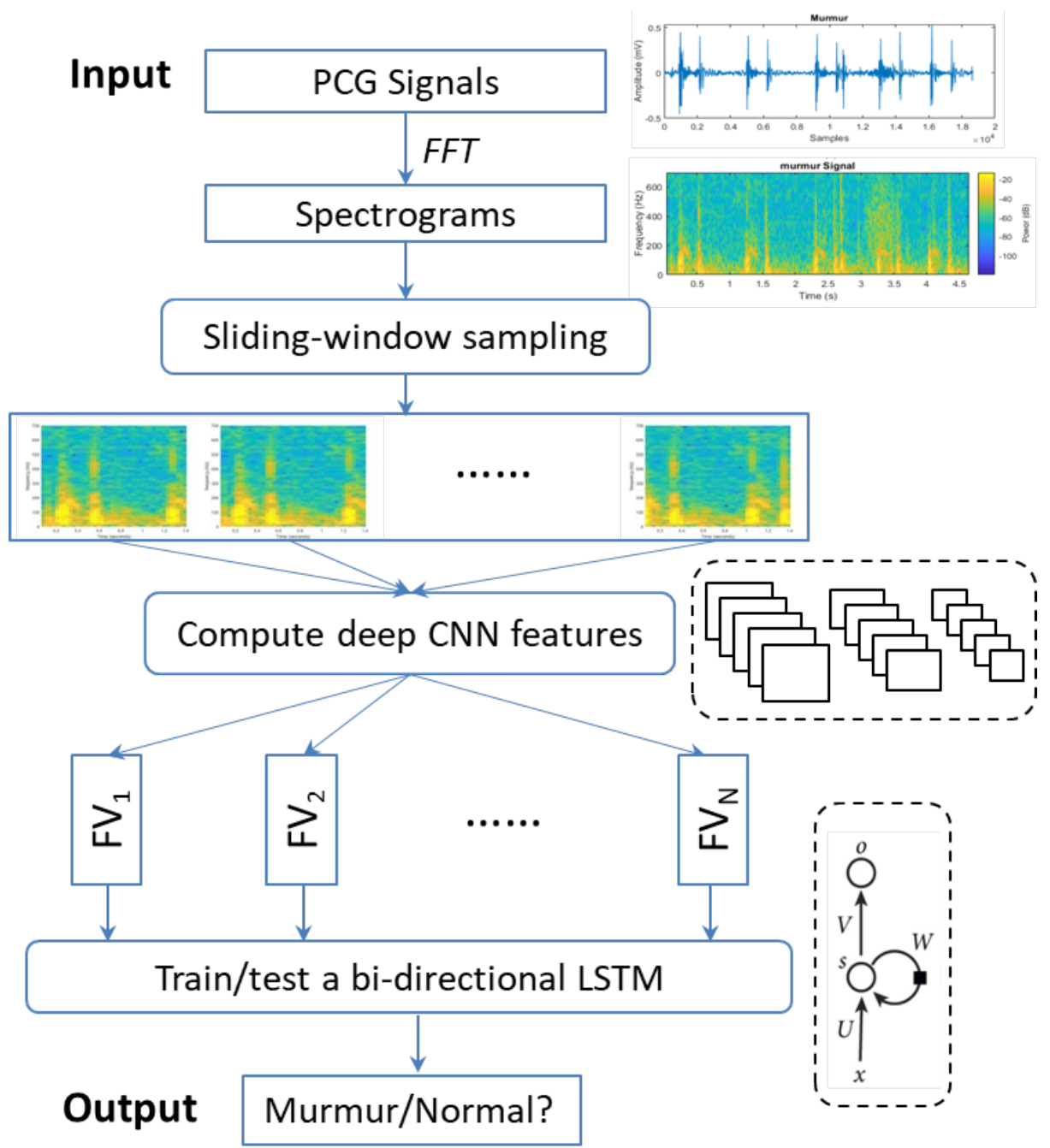

Figure 7: Diagram of a murmur detection approach using deep recurrent CNNs (FFT - Fast Fourier Transform; FV - Feature Vectors). 


\subsection{Experiments with Deep CNNs}

\subsubsection{Comparison on Results from Deep CNN Architectures}

We apply the following parameter settings for training each modified deep CNN: Stochastic Gradient Descent with Momentum (SGDM) as the optimization algorithm, batch size of 10 , initial learning rate as $1 e-4$, and the learning rate for the last fully connected layer as 20.0. Each network stops from further training if the mean accuracy on the fifty most recent batches reaches 99.5\%. On average, the training takes between 20 and 40 minutes to complete on an NVIDIA GeForce GTX TITAN X GPU ( 20 minutes for AlexNet, 40 minutes for VGGNet, and 30 minutes for both GoogLeNet and ResNet). The final size of fine-tuned VGGNet is about 20 times that of GoogLeNet, with in-between sizes for AlexNet and ResNet.

Table 1: Classification Accuracies of Deep CNNs ( $\operatorname{ctep}$ size $=500$ )

\begin{tabular}{|l||c|c|c|}
\hline Model & Sensitivity & Specificity & Overall Accuracy \\
\hline \hline AlexNet & $85.00 \%$ & $90.86 \%$ & $\mathbf{8 7 . 9 3 \%}$ \\
VGGNet & $81.67 \%$ & $89.14 \%$ & $85.40 \%$ \\
GoogLeNet & $95.00 \%$ & $46.29 \%$ & $70.64 \%$ \\
ResNet & $93.33 \%$ & $22.29 \%$ & $57.81 \%$ \\
\hline
\end{tabular}

The voting strategy during testing on a spectrogram is as follows. If over $20 \%$ of the image patches extracted from a spectrogram are classified as murmur, the corresponding PCG is then classified as murmur, otherwise as normal. For each deep CNN, we compute the average accuracies (sensitivity, specificity and overall accuracy) 
from the five-fold cross validations in Table 1. Varying the percentage threshold used in voting makes small updates to the accuracies and they are not big enough in changing the difference among the methods. As an example, increasing the threshold to $60 \%$ leads to overall accuracies for the four models being $86.36 \%, 84.38 \%, 83.02 \%$, and $60.86 \%$, respectively.

Table 2: Classification Accuracies of Deep CNNs ( step size = 100)

\begin{tabular}{|l||c|c|c|}
\hline Model & Sensitivity & Specificity & Overall Accuracy \\
\hline \hline \multirow{2}{*}{ AlexNet } & $81.67 \%$ & $98.86 \%$ & $\mathbf{9 0 . 2 6 \%}$ \\
VGGNet & $76.67 \%$ & $97.71 \%$ & $87.19 \%$ \\
GoogleNet & $98.33 \%$ & $45.14 \%$ & $71.74 \%$ \\
ResNet & $76.67 \%$ & $56.57 \%$ & $66.62 \%$ \\
\hline
\end{tabular}

One natural question is how data augmentation will affect the classification performance of different models. Keeping the rest parameter settings the same, we reduce the window step size from 500 to 100 in order to create more training image patches for both categories of murmur and normal. Because different folds contain PCG recordings at different lengths, we count the average number of murmur and normal training images. The averages increase from $(6,633,9,345)$ to $(13,240,18,617)$ after step size change. Feeding the larger dataset to the four deep CNNs for training and then testing with five-fold cross validations lead to updated performance in Table 2. 

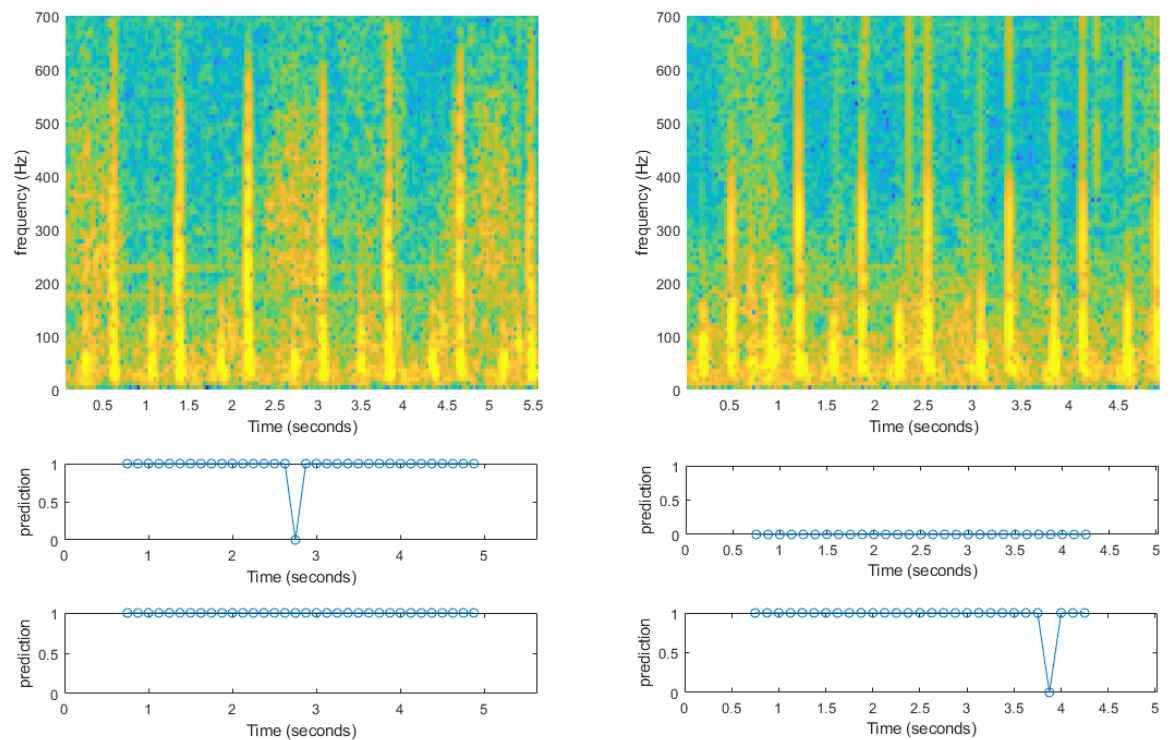

(a)

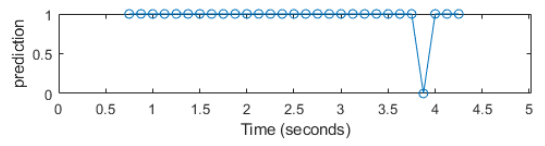

(b)

Figure 8: Predictions on consecutive image patches extracted from spectrograms computed on (a) a murmur PCG and (b) a normal PCG (step size for training image window $=500)$. From top to bottom: spectrogram, prediction from a AlexNet model, and prediction from a ResNet model. 

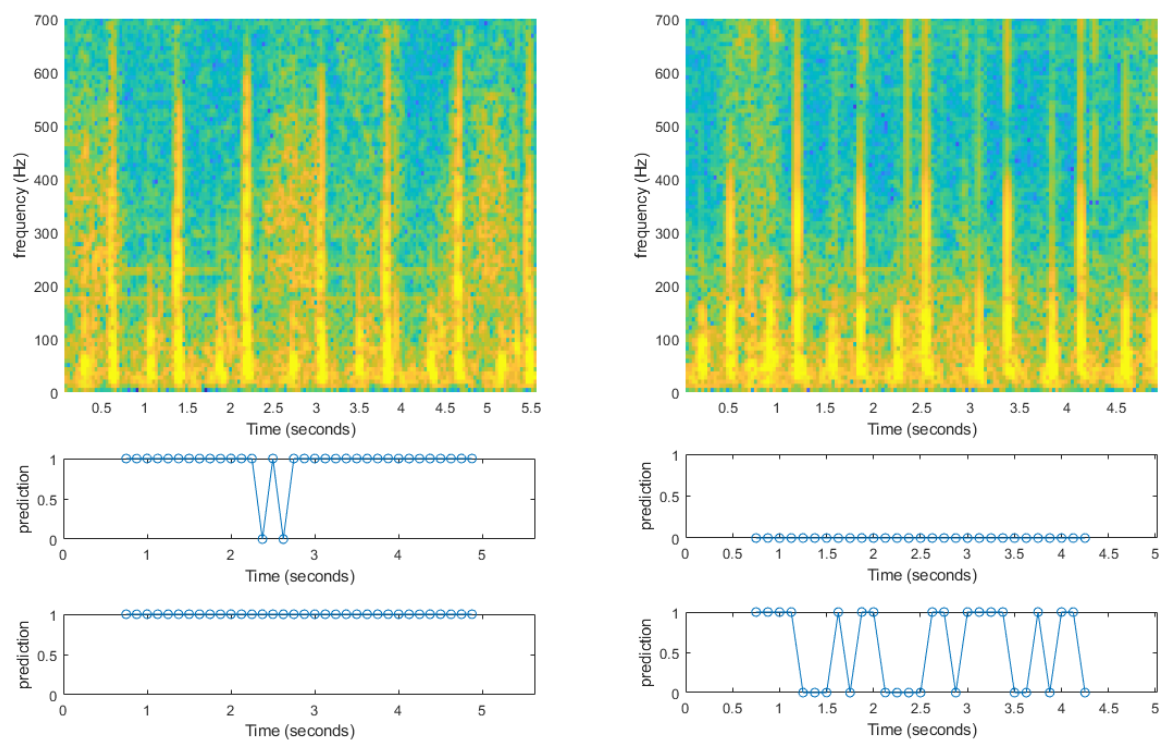

(a)

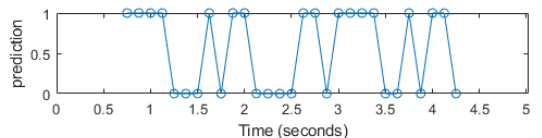

(b)

Figure 9: Predictions on consecutive image patches extracted from spectrograms computed on (a) a murmur PCG and (b) a normal PCG (step size for training image window $=100)$. From top to bottom: spectrogram, prediction from a AlexNet model, and prediction from a ResNet model. 


\subsubsection{Prediction on Spectrograms using AlexNet and ResNet}

We further analyse the performance of different deep CNN models on classifying individual image patches extracted from two spectrograms (one from murmur PCG and the other from normal PCG). In particular, we select AlexNet and ResNet as they represent the best and worst performing models in overall prediction accuracy. We randomly select two spectrograms (one murmur and the other normal) from the independent test set and run classifications with the fine-tuned deep CNNs, which are also randomly selected from one of the five-fold models trained with each architecture.

The first set of prediction results in Fig. 8 are the outputs from deep CNNs trained on image patches extracted at 500 samples apart. In each prediction plot, we use ' 1 ' for output class 'murmur' and ' 0 ' for 'normal'. Their location in the plots matches the center of each sliding window. These plots help identifying the area of the spectrogram that is correctly classified or mis-classified. The left column from top to bottom is the spectrogram for a murmur PCG, prediction results from one of the five-fold AlexNet models, and results from one of the five-fold ResNet Models. The right column of Fig. 8 are the prediction results on a normal PCG.

Fig. 8 indicates that both networks give accurate predictions for the murmur spectrogram, while ResNet predicts poorly on the normal spectrogram (lower right sub-plot in Fig. 8).

Fig. 9 lists the second set of prediction results from deep CNNs trained on image patches extracted at 100 samples apart. Parameter setting for testing remains the same. It indicates that, after data augmentation and model re-tuning, ResNet improves its prediction performance on the same normal spectrogram (lower right sub-plot in Fig. 9). 


\subsubsection{Prediction on Spectrograms using VGGNet and GoogLeNet}

Using the same test examples as previous experiments, we conduct classification experiments on consecutive spectrogram image patches using the fine-tuned VGGNet and GoogLeNet. The classification output results are illustrated in Fig. 10. In comparison with Fig. 9, VGGNet has similar performance as AlexNet in that both make perfect classifications on the normal spectrogram image patches. Also both models make mistakes in classification on two image patches which locate differently in the murmur spectrogram.
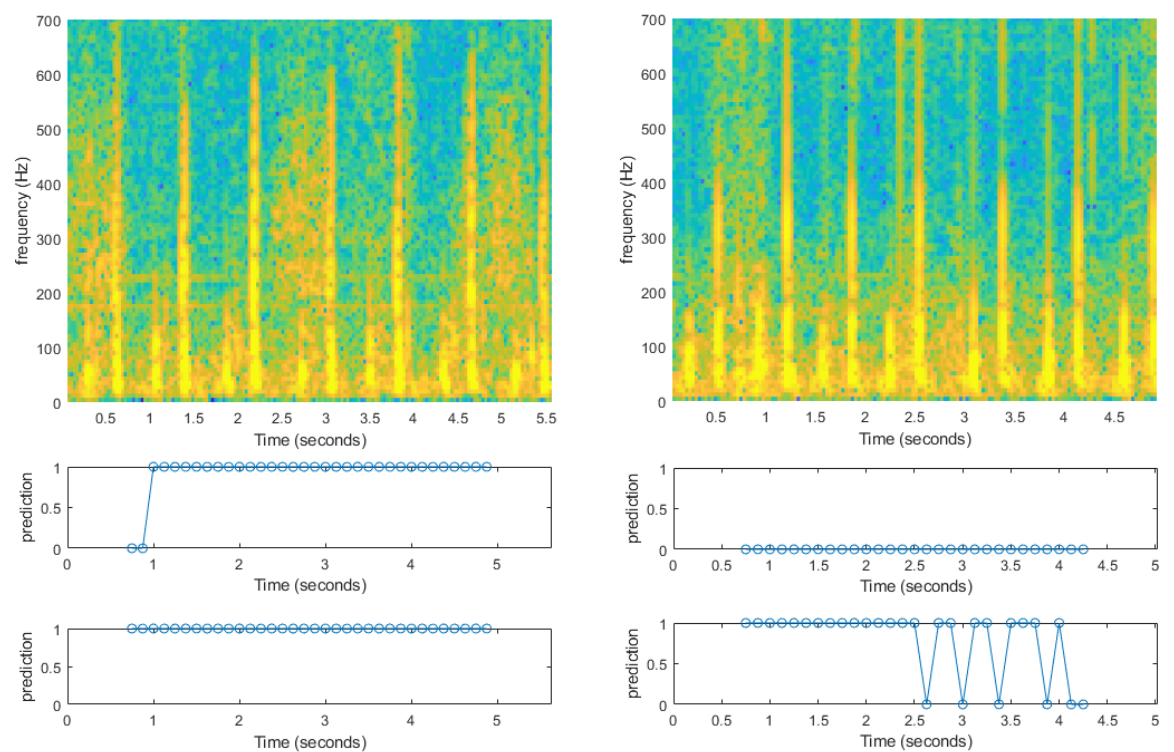

(a)

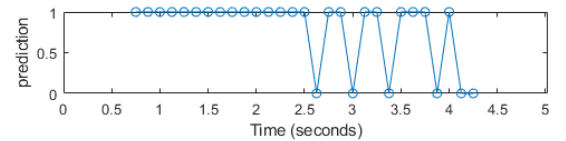

(b)

Figure 10: Predictions on consecutive image patches extracted from spectrograms computed on (a) a murmur PCG and (b) a normal PCG (step size for training image window $=100$ ). From top to bottom: spectrogram, prediction from a VGGNet model, and prediction from a GoogLeNet model.

Likewise, GoogLeNet makes perfect classifications on the murmur spectrogram as ResNet does in Fig. 9. For the normal spectrogram in both figures, ResNet has more 
correct hits than GoogLeNet.

In summary, the comparison in section 3.5.2 demonstrates that increasing the granularity during training image extraction leads to improved performance for ResNet. In both cases, AlexNet gives close to perfect classification result on the two test spectrograms. Maintaining the same granularity as in Fig. 9, we make a further comparison in section 3.5.3 after running classifications with fine-tuned VGGNet and GoogLeNet models. Fig. 10 indicates that VGGNet makes good classification on both spectrograms whereas GoogLeNet has misclassified most of the image patches extracted along the normal spectrogram.

\subsubsection{Using CNN as Off-The-Shelf Feature Extractors}

For comparison, we are studying the use of AlexNet as off-the-shelf feature extractors. At each layer, the portion of the network starting from the input layer is used to extract features from the input spectrogram images. The features are then used to train a two-class support vector machine (SVM). Each SVM model is then validated on the independent testing data set. F-scores are plotted in Figure 11. Overall, the F-score increases over the layers in the network. The largest F-score was achieved at the layers 'relu6' and 'drop6' for $\mathbf{0 . 7 1 .}$

\subsection{Experiments with Deep Recurrent CNNs}

\subsubsection{Use of Spectrogram as Data Representation}

We use the following parameter settings for training the bidirectional LSTM. Adaptive moment estimation (ADAM) solver is selected because it works better with LSTMs than stochastic gradient descent with momentum (SGDM) solver. Learning rate is set at 0.01 with a mini batch size of 50 and max epochs of 200. A gradient threshold 


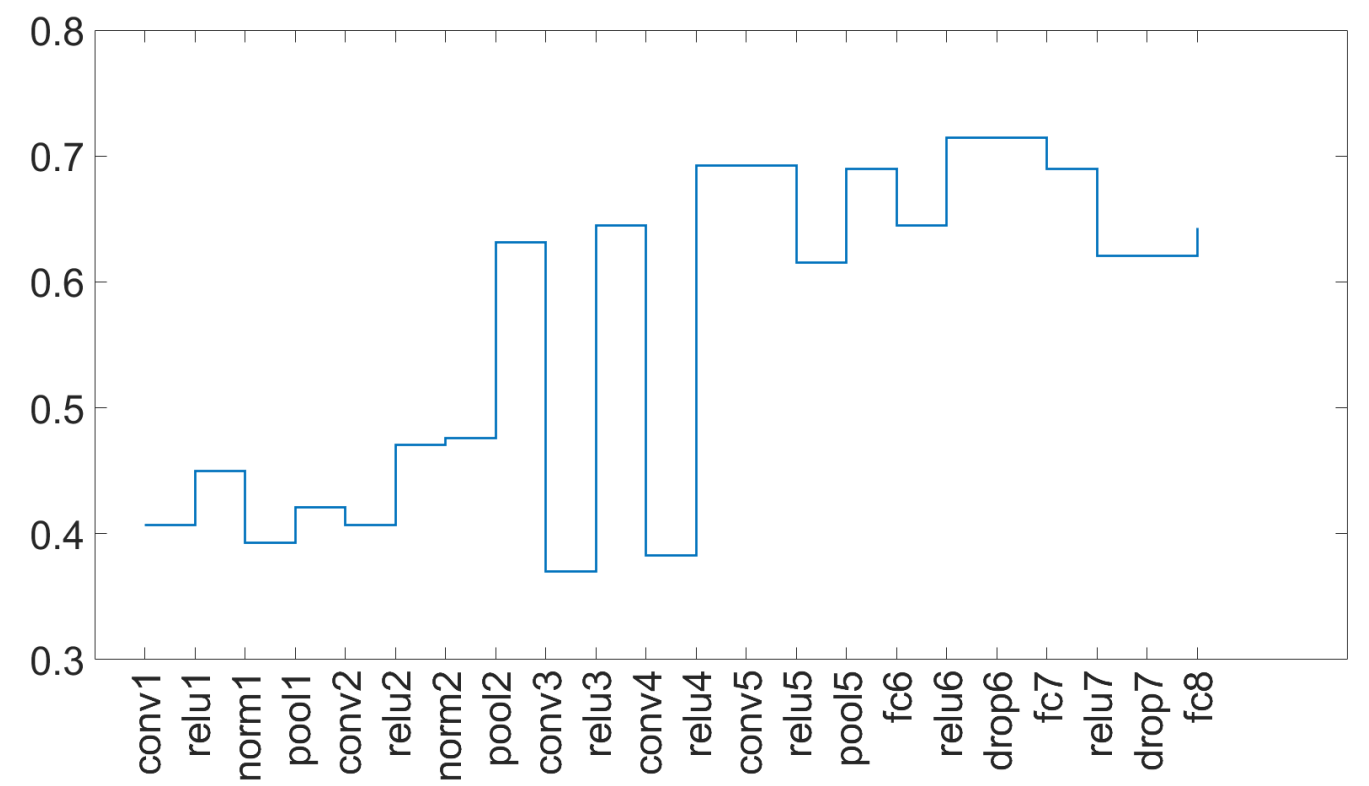

Figure 11: Classification rates (F-score) over different layers in the deep CNN

of 1.0 is also set to stabilize the training process for avoiding large gradients.

We conduct five-fold cross validation on the stability of the trained classifier. Data selection for training and testing is on subject level. In each fold, we put data from twenty percent of the subjects into the testing set and the remaining into the training set. With the training image window step size set at 100, every ten consecutive spectrogram images are sampled and aggregated as one trial. Because PCG signals vary in length from subject to subject, the number of training and testing trials are different in various folds.

Experiments on deep CNNs indicate that AlexNet achives the best performance. Therefore within each trial, every image is fed into the fine-tuned AlexNet model for computing feature tensors (size of $6 \times 6 \times 256$ ). They are computed as the outputs from the last max pooling layer, which is selected based on a previous study [21]. After reshaping the feature tensors into vectors, the bidirectional LSTM starts training and it takes about one hour to complete. 
Table 3: Classification Accuracies of Deep Recurrent CNNs.

\begin{tabular}{|l||c|c|c|}
\hline Fold & Sensitivity & Specificity & Overall Accuracy \\
\hline \hline 1 & $74.69 \%$ & $93.33 \%$ & $85.10 \%$ \\
2 & $100.00 \%$ & $91.12 \%$ & $94.16 \%$ \\
3 & $96.82 \%$ & $99.53 \%$ & $98.38 \%$ \\
4 & $80.21 \%$ & $100.00 \%$ & $93.55 \%$ \\
5 & $98.85 \%$ & $98.90 \%$ & $98.87 \%$ \\
\hline Average & $90.11 \%$ & $96.58 \%$ & $\mathbf{9 4 . 0 1 \%}$ \\
\hline
\end{tabular}

Table 3 lists the classification results from cross-validations. Most folds demonstrate performance boost over those gained in using deep CNNs only. The average of the overall accuracy reaches $94.01 \%$, which is a four-percent improvement over the best result achieved with deep CNNs.

Traditional approaches have been applied to the same data set. These approaches first segment the PCG signals, extract features and then build classifiers. Two representative methods achieved a precision below $40 \%$ on detecting murmurs [65] [66]. A recent work from 2018 [67] boosted the performance to about 71\%. A complete comparison on the results are listed in Table 4. The features used for training the traditional classifiers comprise those in the time domain, including zero crossings, energy and entropy of energy, and those in the frequency domain, including spectral spread, spectral entropy, spectral flux and Mel Frequency Cepstral Coefficients (MFCCs) [67]. 
Table 4: Comparison on Classification Accuracy with Traditional Approaches.

\begin{tabular}{|l||c|c|c|c|c|c|}
\hline Meas. & Naive & SVM & Decis. & Ada- & Random & Gradient \\
& Bayes & & Trees & Boost & Forest & Boosting \\
\hline \hline F-score & 0.45 & 0.69 & 0.68 & 0.46 & $\mathbf{0 . 7 1}$ & 0.67 \\
\hline
\end{tabular}

\subsubsection{Use of Scalogram and Mel Spectrogram}

Besides the spectrogram used in this study, we are computing different representations of the digital signals for a comparison. More specifically, we are computing scalogram and mel spectrogram and train the deep Recurrent CNNs on them.

A scalogram is a visual representation of a wavelet transform, having axes for time, scale and coefficient value, analogous to a spectrogram. The fixed window size used in spectrogram limits the time-frequency resolution. In contrast, wavelet transform uses variable sized windows to capture features occurring at different scales. These scaled wavelets are shifted along the entire length and compared with the signal. As a result, high frequency events are better resolved in time and low frequency events are better resolved in frequency.

A mel spectrogram is also a visual spectrum of a signal and it uses a mel filter bank for the analysis. The mel filter bank is designed as half-overlapped triangular filters equally spaced on the mel scale. These filters are non-uniformly spaced on the frequency axis, with more filters in the low frequency regions.

\section{Data Pre-processing}

For illustration purposes, we randomly select a normal and a murmur PCG, extract the first three seconds, and compute the selected time-frequency representations. The 
visual outputs are demonstrated in Fig. 12. To compute spectrograms, we applied a 512-point Hamming window with 75\% overlapping for computing Fast Fourier Transform (FFT) of size 512. The limits of the color axis for spectrograms are set to be between $-120 \mathrm{db}$ and $-40 \mathrm{db}$. To compute scalograms, we apply continuous wavelet transform (CWT) on the PCG signal. The magnitude of the color axis for scalograms are set to be between 0 and 0.03 . To compute mel spectrograms, we applied a 512point window with $75 \%$ overlapping for computing Fast Fourier Transform (FFT) of size 512. The number of bands is set at 54. The limits of the color axis for spectrograms are set to be between -40db and 10db.

\section{Study on the Influence of Temporal Features}

The first set of experiments are to compare the different time-frequency representations while fine-tuning an AlexNet model. The second set of experiments are conducted to study the influence of temporal features after integrating the AlexNet with an LSTM model. After conducting five-fold cross validations, the average F-Scores are listed in Table 5.

Table 5: Classification Accuracies (F-Score) using Different Features

\begin{tabular}{|l||c|c|c|}
\hline Features & Spectrogram & Scalogram & Mel Spectr. \\
\hline \hline Spatial and Spectral & 0.8775 & 0.8965 & $\mathbf{0 . 9 1 2 7}$ \\
\hline Spatial, Spectral and Temp. & 0.9540 & 0.9680 & $\mathbf{0 . 9 7 6 7}$ \\
\hline
\end{tabular}




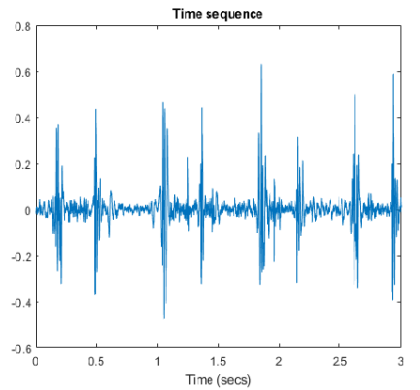

(a) Murmur case, raw signal

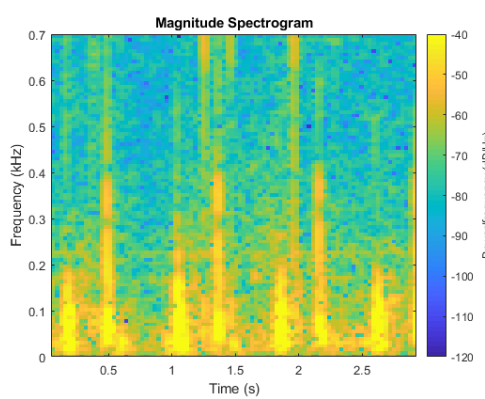

(c) Murmur case, spectrogram

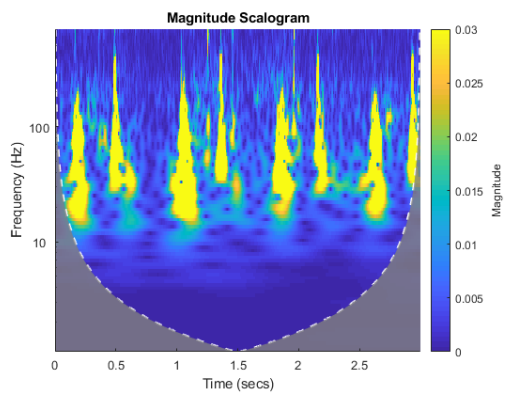

(e) Murmur case, scalogram

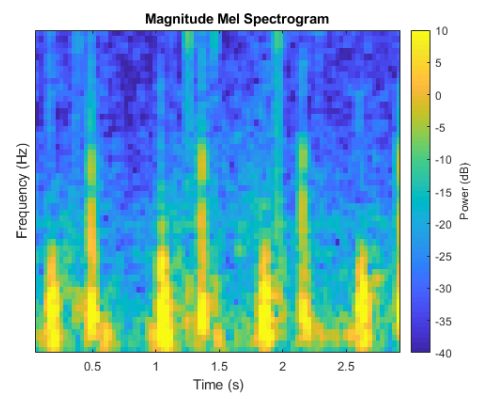

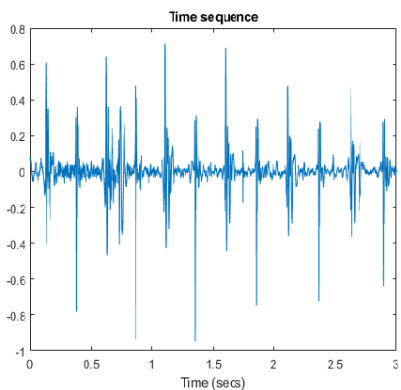

(b) Normal case, raw signal

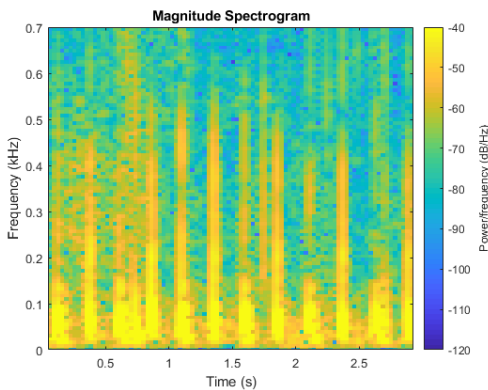

(d) Normal case, spectrogram

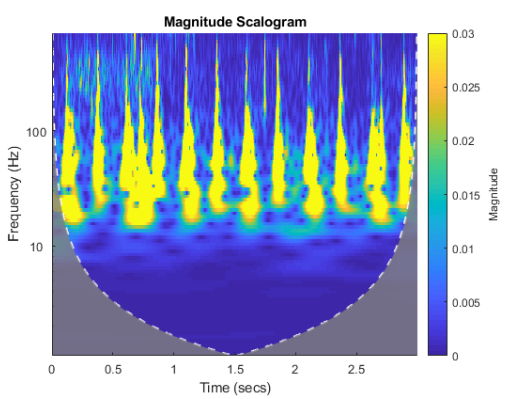

(f) Normal case, scalogram

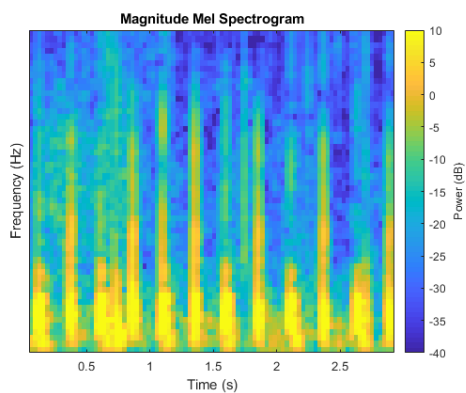

(g) Murmur case, mel spectrogram (h) Normal case, mel spectrogram

Figure 12: Different data representations for a murmur case (a, c, e, and g) and a normal case (b, d, f and h). Both cases are three seconds in duration. 


\section{Visualization of Features using $t$-SNE}

In order to visualize the features in both categories, we use $t$-distributed stochastic neighbor embedding ( $t$-SNE), an algorithm for dimension reduction that is well-suited to visualizing high-dimensional data [68]. It embeds high-dimensional points in low dimensions and retains the similarities between data points. We select one of the layers in the fine-tuned AlexNet, compute feature outputs from the layer and apply $t$-SNE for visualization. Figure 13 illustrates this by plotting the 2D mapped features from 'conv4' layer in the AlexNet model trained on spectrograms.

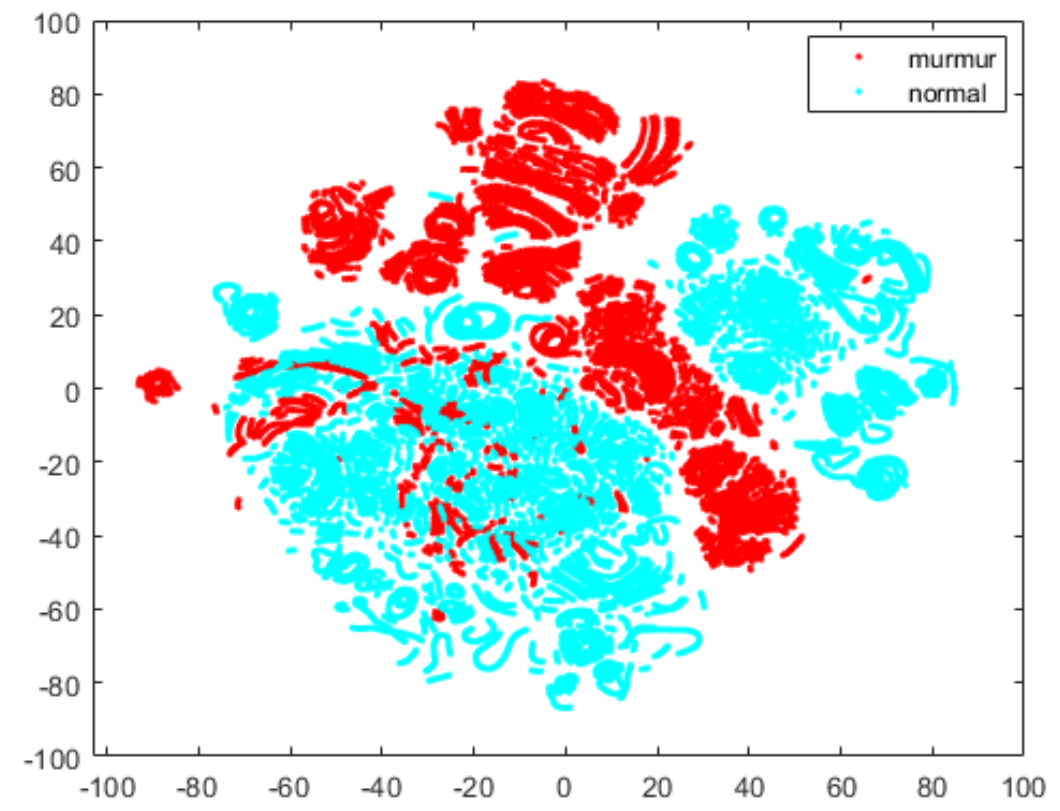

Figure 13: Plot extracted features using spectrograms as input.

The layer 'conv4' is the second last convolutional layer in the AlexNet model. From Figure 13, one can see clear separations between the murmur and normal classes. In fact, the continuous curves are interesting findings because they match the continuous representations in the original data space (consecutive sampling of spectrograms along the time sequence). 


\subsection{Discussion}

The four deep CNN models in comparison demonstrated consistent improvement on the ILSVRC benchmark tasks; however, the same trend does not hold in the cardiac murmur classification task. Our explanation is that deeper models (ResNet being the deepest among the four) have more complexity and parameters, therefore they are more data thirsty in fine tuning. This has been verified through our experiments in which data augmentation with denser image patch extractions leads to improved performance of the deep CNNs, most notably for ResNet.

We use a voting strategy during testing. In a different strategy, the probability of a PCG recording being classified as either murmur or normal can be computed as the average probabilities assigned to its randomly selected window patches.

The comparisons are conducted on two randomly selected testing spectrograms with randomly selected fine-tuned deep CNN models. With a different selection on both testing data and models, the results will be slightly different but the observed difference should remain the same.

Besides the visual features extracted from deep CNNs, we have also experimented with simpler features extracted from spectrograms. Among many, we computed two time-frequency ( $\mathrm{TF}$ ) moments, namely instantaneous frequency and spectral entropy, which are in itself one dimensional. We then fed them to the bidirectional LSTM for training. Experimental results show that these features led to an overall accuracy at around 75 percent, much lower than those achieved with deep CNNs. This is another proof of the power from visual feature extractions through deep CNN models.

Overall, our approach on using deep recurrent CNNs demonstrates performance boost over the method on using deep CNNs only. The majority of the cross validation folds demonstrates an absolute performance gain over the deep CNN approach. This indicates that temporal features captured with LSTMs is a great addition to the 
spatial and spectral features learned with deep CNNs.

\subsection{Conclusion}

The classification between cardiac murmur and normal PCGs is a challenging problem because of subtle differences. This work successfully employs deep CNNs with transfer learning and data augmentation to learn from a small dataset of PCG recordings. The power of deep CNNs on automatic feature extractions enables accurate classifications without the need of segmentation. Four state-of-the-art deep CNN architectures are compared on classification performance.

In the ILSVRC benchmark tasks, deeper models showed higher performance than their counterparts with less number of layers. Training with the limited PCG dataset, the deeper models do not share the same performance gains. In fact, the least deep model in selection demonstrates better performance in all comparisons. Fine tuning the deep CNN models is data thirsty and data augmentation improves performance for all the deep CNN models. The result can be used as a guidance for deep model selections in a similar problem given limited amount of training data.

Adding to the spatial and spectral features computed with deep CNNs, we improve our approach using LSTMs to extract temporal features. Experimental results indicate a performance boost after including the temporal features. After conducting comparisons on three time-frequency representations, mel spectrograms achieve the best F-score of 0.9767, using the fine-tuned AlexNet with the LSTM network.

In conclusion, we validate the proposed deep learning framework on 1-D physiological data. We demonstrate the framework using deep recurrent convolutional neural networks to extract spatial, spectral and temporal features from PCG signals. Experimental results indicate that the deep recurrent $\mathrm{CNN}$ based approach is a practical solution to the detection of cardiac murmurs. 


\section{Chapter 4}

\section{Representation Learning on 2D Medical}

\section{Images}

Although deep CNN models are mostly designed for 2D images, they often only work with small size images. In applications involving high-resolution images such as mammography, challenges arise when applying existing deep CNN models. Therefore, this chapter introduces the proposed deep representation learning framework for this type of applications. The validations are conducted on a mammogram data set and a ultrasound liver image data set. In the proposed deep learning framework, components used for the validation are highlighted in Figure 14.

Computer aided detection of abnormalities in medical images has clinical significance but remains a challenging research topic. Despite recent success of deep learning in computer vision, achieving the same success on medical images is not trivial because of the insufficiency of training data and the lack of deep neural network architectures designed for medicine. Moreover, training deep convolutional neural networks often compresses high-resolution images at input layer to a set size, causing the trained detectors insensitive to medical abnormalities usually found in tiny local areas of high-resolution images. Traditional approaches use manually designed features to 

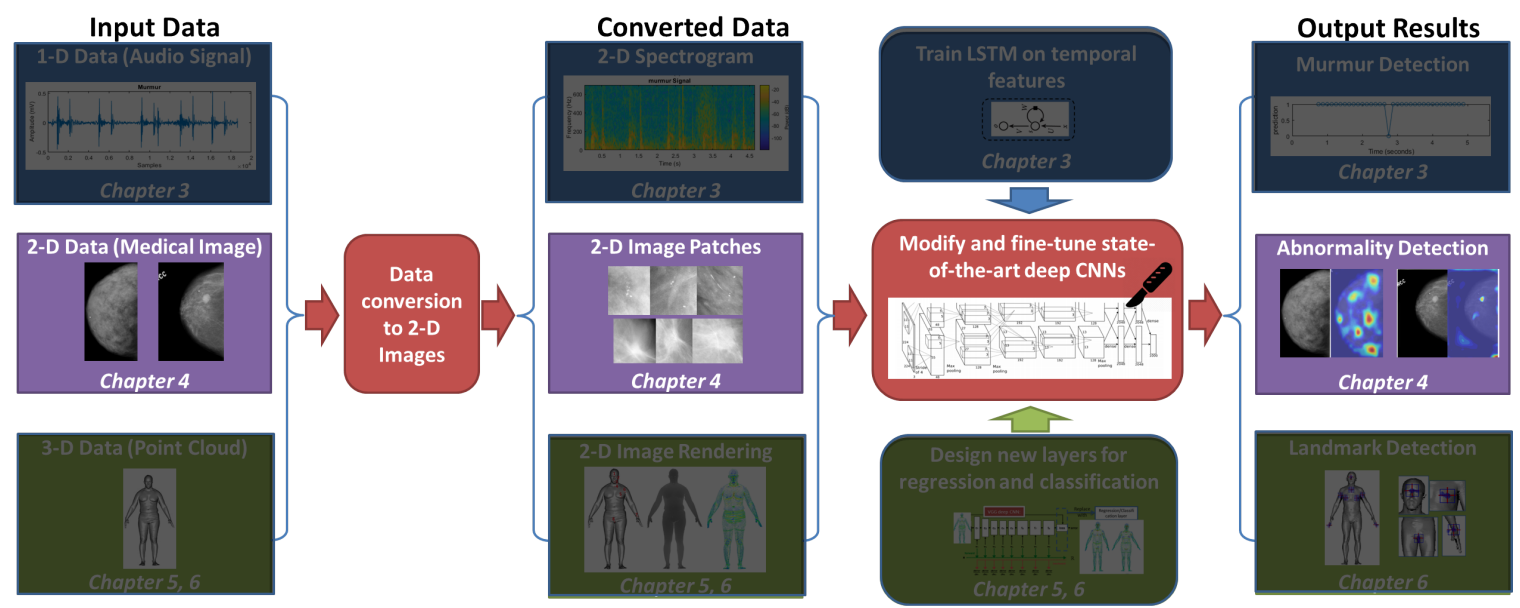

Figure 14: Applying the deep representation learning framework on 2D medical image data (from left to right: raw data, converted 2D data representations, deep model training and output).

build sliding window-based detectors, and therefore they have limitations on both efficiency and accuracy. To address these challenges, we propose transfer learning-based classifiers trained on image patches centered at medical abnormality, and build deep detectors through the integration with class activation mappings and region-based convolutional neural networks. The detectors are tested on a mammogram data-set and an ultrasound liver image data-set. In both cases, the proposed deep learning architectures demonstrate superior performance over the traditional approaches. It also indicates that the integrated approaches will provide better outcome to patients, in assisting doctors on detecting abnormalities from medical images.

\subsection{Introduction}

Medical imaging uses different modalities and processes to image the human body for diagnosis and treatment of disease. Radiologists use medical images to identify 
abnormal tissues, conduct measurements, and provide reports to doctors for diagnosis. Over the years, computer aided detection (CADe) has played an increasingly important role in assisting the medical professionals. CADe is a pattern recognition process that detects potential abnormalities in medical images.

CADe of medical abnormality is a challenging process because of low contrast and high noise levels in medical images. In particular, what makes it a unique problem is that medical abnormalities often appear in tiny local areas within a high-resolution medical diagnostic image. For example in mammography, benign calcifications tend to be large and do not require magnification; however, the suspicious ones tend to be smaller and their characterization needs to be studied with magnified images [69].

Traditional CADe approaches build sliding window-based classifiers to inspect medical images for detecting abnormalities [70] [71]; however, these approaches have issues with efficiency because of redundancy in computing with the overlapped windows, and with accuracy because of non-optimal settings of window and step sizes. Another drawback of the traditional approaches is that they depend on manually designed features. This feature engineering process relies on domain knowledge and is known to be tedious, time consuming and error prone.

In comparison, recent advances in deep convolutional neural networks (CNN) have enabled automatic feature learning [6] [7] [8] [9]. Because of these advances, deep CNNs have emerged as a go-to solution in general object detection. However, an obstacle for applying the deep CNNs is the need of large amount of training data [72], whereas medical applications are usually low with training data. Therefore, it remains a challenging problem in making effective use of deep CNNs for abnormality detection in medical images.

Recently, deep learning has been applied to medical image classification and detection [73]. Wang et al. [74] introduced ChestX-ray8, a hospital-scale chest X-ray 
database, and provided benchmarks on weakly-supervised classification and localization of common thorax diseases. Following this work, Rajpurkar et al. [75] introduced CheXNet, a 121-layer dense convolutional network (DenseNet) trained on the ChestX-ray 14 data-set, producing radiologist-level pneumonia detection. In addition, Rajpurkar et al. [76] introduced MURA data-set for detecting abnormality in musculo-skeletal radio-graphs. Rather than inventing deep CNN architectures for the newly created medical data sets, these approaches re-used and fine-tuned existing CNN architectures that had been trained on natural image data set, such as ImageNet Large Scale Visual Recognition Competition (ILSVRC) [2]. When re-using the existing deep CNNs, all the medical images were scaled to $224 \times 224$ (width and height in pixels) at input layers in order to train the networks. This may not be a problem for the above tasks, but the image compression leads to loss of information that is crucial for abnormality detection such as in mammography.

In addressing the challenges found with the traditional methods, we propose an approach based on automatic feature learning. Once trained, the model undergoes a one-pass forward computation to create an abnormality map for a test image. This significantly improves the efficiency on detecting abnormality. In addressing the information loss problem found with the deep learning work, we train models on cropped image patches centered at medical abnormality, and fine-tune them for detecting abnormality in full images through leveraging state-of-the-art object detectors.

Our approach starts from training deep CNNs on image patches centered at medical abnormalities. During training, transfer learning is used to avoid over-fitting, because of the limited number of training images. In applications where the training set only provides abnormality patches, the proposed approach integrates the deep patch CNNs with class activation mappings (CAM) [77] for computing an abnormality map from a full test image. In other applications where both full images and patch locations are available during training, the proposed approach integrates 
the deep patch CNNs with region proposal networks (RPN) for fine-tuning a faster region-based CNN (R-CNN) [72].

To evaluate the proposed approach, we use a mammogram data-set [78] and an ultrasound liver image data-set, both in limited quantities. Experimental results indicate that the proposed approach yields better performance than traditional approaches on both image patch classifications and abnormality detection.

We have made the following contributions:

- Successful integration of pre-trained deep patch CNNs with CAMs for detecting local abnormalities from high-resolution medical images;

- Effective fine-tuning of faster R-CNN with limited medical data for abnormality detection using transfer learning;

- Demonstration of performance boost from deep CNN-based detectors over traditional approaches.

This chapter is organized as follows. In Section 4.2, we review recent research work in computer aided detection of abnormalities. We then introduce the proposed approaches in Section 4.3, followed by experimental results in Section 4.4. After discussions in Section 4.5, we summarize the work in Section 4.6.

\subsection{Related Work}

Traditional CADe approaches have reached performance plateau before deep learning emerges as an end-to-end solution for both feature extraction and classifier building. However the adoption of deep learning in CADe is limited by the insufficiency of training data and the lack of customized deep neural network architectures. This section reviews both traditional and deep learning-based CADe techniques. 


\subsubsection{Traditional CADe Approaches}

In mammography screening, common abnormalities include micro-calcification (MC) and mass, which are usually found in tiny local regions within a high-resolution medical image. Most CADe approaches for calcification detection follow a common procedure: image enhancement, segmentation or extraction of region of interests (ROIs), feature computation and classification. For mass detection, algorithms first identify suspicious regions in a mammogram and then classify it as mass or normal tissues.

Micro calcifications are tiny deposits of calcium that appear as bright spots in mammograms. Filter banks were used to decompose mammogram images followed by ROI selection and Bayesian classifications [79] [80]. Pal et al. [81] introduced a multistage system for detecting MCs in mammograms. They used a back-propagation neural network to find candidate calcification regions, cleaned network output to remove thin elongated structures and used a measure of local density for final classification. Similarly, Harirchi et al. [82] applied a two-level algorithm for the detection of MCs using diverse Adaboost support vector machine (SVM). Six features (four wavelet plus two gray level features) were computed for neural networks to detect candidate MC pixels. As a result, 25 features from candidate MCs were extracted and further reduced with geometric linear discriminant analysis (GLDA). The classifier was built with diverse Adaboost SVM. Oliver et al. [83] extracted local features to train a pixelbased boosting classifier, which at each round automatically selects the most salient micro-calcification features. Zhang et al. [84] enhanced the MCs using well-designed filters and then conducted subspace learning for feature selection. A twin SVM was used for classification.

A mass in mammogram is defined as a space-occupying lesion seen in more than 
one projection [85]. The general procedure for detecting mass is first to detect suspicious regions, then extract shape and texture features, and finally detect mass regions through classification or removing false positive regions [86]. Petrosian et al. [87] used texture features to distinguish mass and non-mass regions. Petrick et al. [88] used an adaptive density-weighted contrast enhancement filter to obtain potential masses and used Laplacian Gaussian for edge detection. Morphological features were extracted for classifying normal and mass ROIs. Cascio et al. [89] segmented the boundary of ROI using an edge-based approach and computed geometric and shape features. Classical neural networks were trained to distinguish true mass from normal regions.

\subsubsection{Deep Learning for CADe}

Deep learning has been applied to breast lesion detection [90] [91]. In [90], the authors exploited deep learning for differentiating the types of lesions and nodules. A biopsyproven bench-marking data-set was built on semi-automatically segmented lesions. GoogLeNet-based CNN was trained for the classifications. The drawback of the system is that it needs radiologists to select target ROIs on new images. In [91], the authors proposed deep learning approaches for breast lesion detection and compared them with traditional lesion detection algorithms.

Ultrasound (US) images are usually monochrome and low-resolution. In US images, cancer regions are usually blurry, with vague margin and irregular shape. Moreover, the feature of cancer regions are very similar to normal or benign tissues. Li et al. [92] introduced a deep learning approach for the detection of thyroid papillary cancer in US images. They added a spatial constrained layer to CNN so that the detector can extract the features of surrounding region in which the cancer regions are residing. Another deep learning approach in detecting thyroid nodules in US images is introduced in [93], where a pre-trained GoogLeNet model is fine-tuned for feature extraction. The features are then fed to a random-forest classifier to classify 
the images into "malignant" or "benign".

Deep learning has also been applied to segmenting important structures from medical images [94] [95]. Fu et al. [95] applied multi-scale and multi-level CNN with a side-output layer to learn hierarchical representation of features. To model the longrange interactions between pixels, Conditional Random Field (CRF) is used. The proposed method achieved state-of-the-art performance for retinal vessel segmentation. In [94], Fu et al. proposed a new deep learning architecture for segmenting optic disc (OD) and optic cup (OC) from fundus images. The new architecture comprises multi-scale input layer, U-shape convolutional network, side-output layer and multi-label loss function. The structure solves the OC and OD segmentation jointly in a one-stage multi-label system.

In summary, the traditional CADe approaches rely heavily on manual features, which are known to be time-consuming and error-prone. In deep learning CADe, to our best knowledge, we are the first in integrating the deep patch CNNs with CAMs. We are also the first in applying deep CNNs onto ultrasound liver images.

\subsection{Methodology}

Abnormality detection in medical sensing images is essentially an object detection problem; however, applying general object detection techniques to the medical domain remains a challenge. Traditional object detectors rely on manually designed features for training the classifiers, whereas deep learning-based detectors need a large amount of training data. Different from natural image applications, medical abnormality detection poses new challenges. In applications such as mammography, there is a unique problem that the abnormalities are located in tiny local regions within a highresolution medical image.

In order to address the challenges while solving the unique problem, this work 


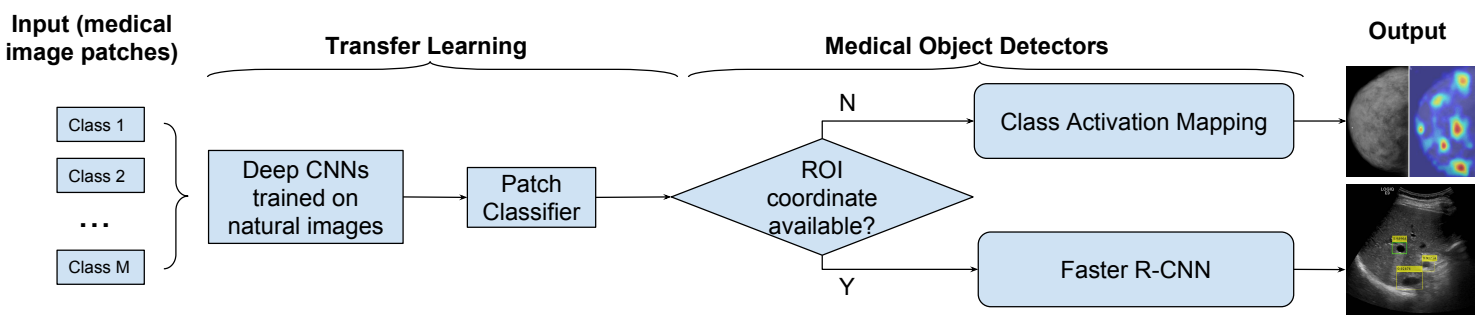

Figure 15: Diagram of an integrated approach for medical abnormality detection using deep convolutional neural networks.

proposes an approach by training transfer learning-based patch classifiers, which are then integrated with class activation mappings and region proposal networks. The patch classifiers serve as an automatic and effective feature extractor for the integration. With class activation mapping, the proposed approach is able to compute an abnormality map for a test image through an one-pass forward computation, avoiding the drawbacks on efficiency and accuracy from sliding window-based approaches. The integration with region proposal networks shares deep convolutional features with the deep patch classifiers, and it leads to a highly efficient and accurate approach for medical abnormality detection. Performance is compared with traditional detectors on a mammogram data-set and an ultrasound liver image data-set.

The proposed approach is illustrated with Figure 15. It starts with transfer learning to deal with limited training data. In order to learn image features for detecting the local abnormalities, image patches centered at medical abnormalities are collected for fine-tuning deep CNNs that have been pre-trained on natural images. In applications where only image patches are available during training, the proposed medical object detector integrates the patch classifiers with class activation mapping. Once trained, the CAM-based detector is able to compute an abnormality map over a full medical image. In other applications where both full images and the coordinates of 
image patches are available during training, the object detector integrates the patch classifiers with region proposal networks to build and fine-tune a faster region-based CNN. Once trained, the R-CNN detector is able to output abnormalities labeled with rectangles on a test image.

\subsubsection{Abnormality Detection with Class Activation Mapping}

Computer-aided detection of mammogram abnormalities cannot be simply treated as an image classification task, because the abnormalities are located in tiny local regions of a high-resolution image. For example, a typical mammogram image with resolution $3000 \times 4600$ (width and height in pixels) contains an abnormality region of size at about $200 \times 200$ (in pixels) or less. Training recent deep CNNs requires resizing images to $224 \times 224$ (in pixels) at the input layer, causing massive loss of information about the medical abnormalities. Consequently this makes it difficult to train on full images for detecting the local abnormalities. To deal with this challenge, we train deep CNNs on cropped image patches (the ROIs) and adapt them to full mammogram images.

Figure 16 illustrates the data-flow of our approach. With training image patches from calcification and mass cases, a two-class classifier is trained with state-of-the-art deep CNN architectures using transfer learning (step 1) [14]. The pre-trained CNNs are modified at output layers to have two output classes. The output layers are then fine-tuned while the first part of the network is frozen.

The fine-tuned patch neural network is then used to localize abnormalities in fullsize mammograms. Traditional approaches used the classifier to scan the whole image with a sliding window and suffered on efficiency [14]. In contrast, our approach enables localizing abnormalities in one single forward pass of computation. Feeding the full-size mammogram image into the patch classifier and computing class activation mappings [77] produce a heat-map on abnormalities. 


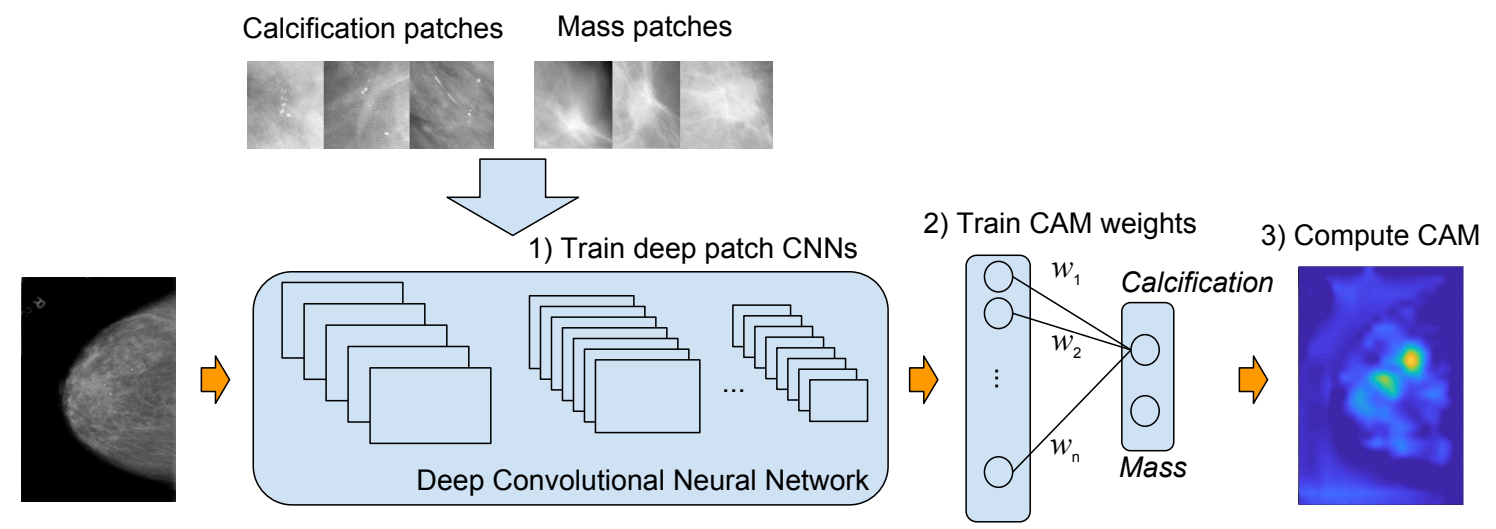

Figure 16: Diagram of CAM-based approach: 1) train deep CNNs using abnormality patches; 2) modify network and compute the weights for CAM; 3) given a test image, compute color-coded abnormality map using CAM.

CAM is a technique for identifying regions in an image using a CNN for a specific class [77]. In other words, CAM identifies image regions relevant to a class. It allows re-using classifiers for localization purpose, even when no training data on locations have been made available. It also demonstrates that CNNs have a built-in attention capability.

Training and computing CAM for mammograms are explained with steps 2 and 3 in Figure 16. A deep CNN needs to be cut after the last convolution layer, and a global average pooling layer and a fully connected layer are appended. The new model needs to be re-trained for learning the CAM weights $w_{i}(i=1,2, \ldots n)$ at the output layer. Among the four deep CNN architectures, the ResNet is ready for computing CAM after feeding a full mammogram at the input layer. The feature maps (in tensor forms) from the output of the last convolutional layer are denoted as $F_{i}(i=1,2, \ldots n)$. We can identify the importance of the image regions by projecting back the weights of the output layer onto the convolutional feature maps [77] through: 


$$
C A M=\sum_{i=1}^{n} w_{i} F_{i}
$$

The output CAM is then displayed for visualization and verification.

\subsubsection{Abnormality Detection with Faster R-CNN}

Despite speed up in traditional approaches [96] [97] [98], the past several years witnessed tremendous progress on object detection using deep learning, including:

- R-CNN [97] - this approach combines rectangle region proposals and convolutional neural networks. The first step is to compute a set of regions that may contain objects and the second step learns classifiers to classify the regions. The region proposals are generated using algorithms such as edge boxes. The proposals are then cropped and resized to train CNN classifiers. Deep CNNs are used for feature extraction to be plugged into a support vector machine to refine the region proposal bounding boxes.

- Fast R-CNN [99] - this approach is similar to R-CNN in that it uses algorithms such as edge boxes to generate region proposals. Instead of cropping the proposal regions and resizing them, this approach works on the entire image by pooling CNN features corresponding to each region proposal. This is a more efficient implementation because it shares computation with overlapping regions.

- Faster R-CNN [72] - this approach makes further improvement by discarding external algorithms such as the edge boxes. It merges the step of region proposal into the entire network and makes computations more efficient.

The three methods share the same component of R-CNN for object detection. Faster R-CNN makes improvements over the other two in that it does not rely on 
external approaches for computing region proposals, which have been seen as a bottleneck. After the faster R-CNN work, several object detectors haven been invented for improving efficiency, such as single shot detector (SSD) [100] and region-based fully convolutional neural networks (R-FCN) [101]. However, a recent study demonstrates that faster R-CNN is competitive on accuracy among the three [102]. It also suggests that, using fewer proposals, faster R-CNN can speed up significantly without a big loss in accuracy. Therefore in this work, we choose faster R-CNN as the detector, and apply transfer learning to adapt it to the medical data-sets. The feature computing of a faster R-CNN can be designed from scratch or borrowed from state-of-the-art deep CNN architectures, such as AlexNet, GoogleNet or ResNet. Because of proven advantages from transfer learning, we experiment on modifying the pre-trained deep CNNs for constructing a faster R-CNN.

Figure 17 introduces the two parts involved in modifying state-of-the-art deep CNN models for building a faster R-CNN. Using ResNet as an example, the first part of modification is performed after one of the convolutional feature extraction layers. After cutting the connections at layer 'activation_40_relu', a new branch for region proposal classification and regression is produced. Both sub branches are linked to a region proposal layer, which is then connected to a new ROI pooling layer. The output of the ROI pooling layer is fed into the previous layers that were attached to 'activation_40_relu'.

The second part is making changes to the output layer. It demonstrates the process of removing original layers after layer 'avg_pool' and attaching two newly created branches for conducting classifications and regressions on region proposals. This completes the network modification for building a faster R-CNN object detector.

Training a faster R-CNN comprises alternate steps between training an RPN and a fast R-CNN, because the latter two share feature computations. The process starts from training an RPN and uses it to train a fast R-CNN. The weights from the fast 


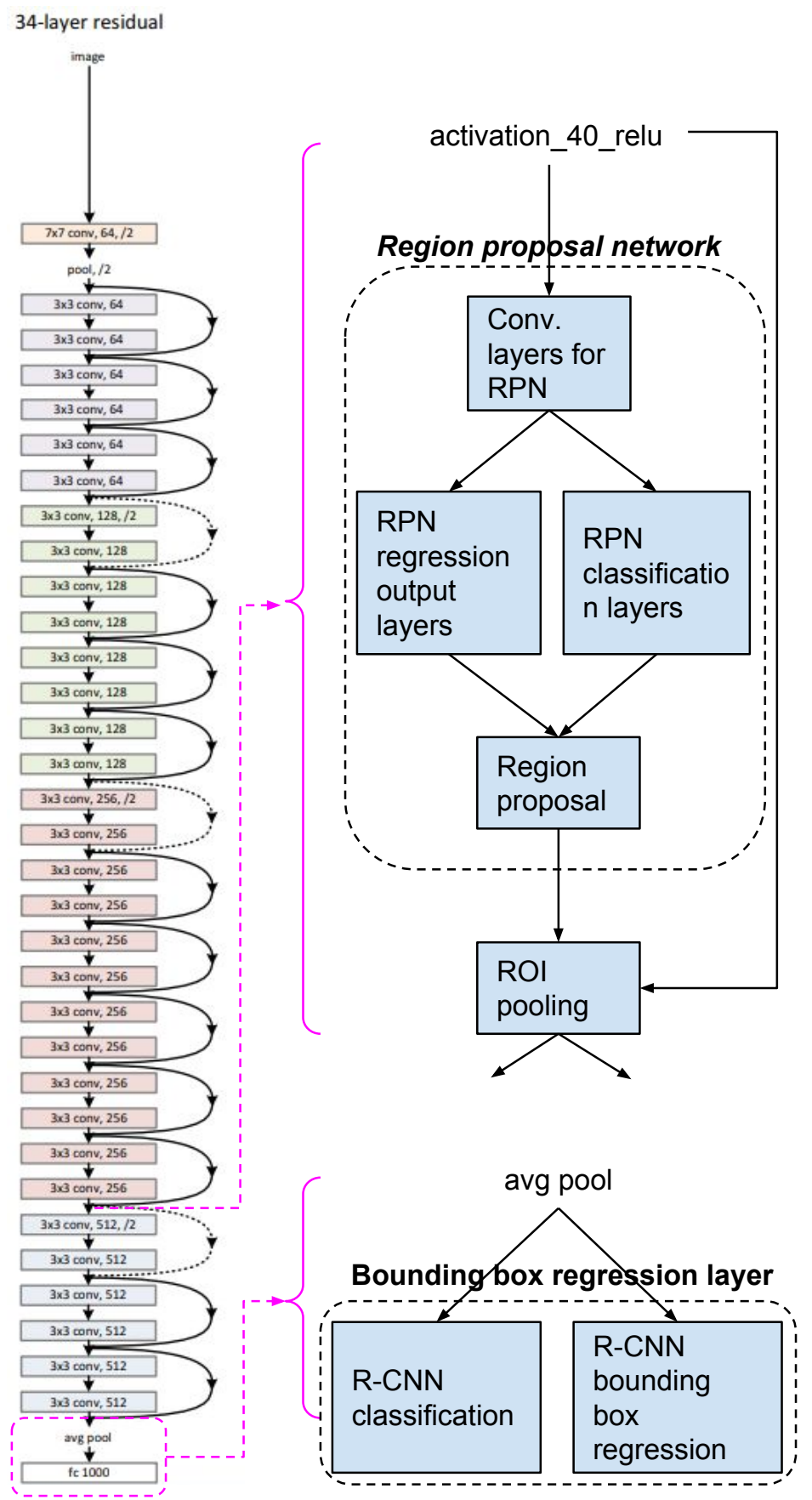

Figure 17: Diagram of R-CNN-based approach: ResNet is cut at a convolution layer and a region proposal network is added; bounding box classification and regression layers are used to replace the original output layer of the ResNet. 
R-CNN are then shared with re-training the RPN. The updated RPN is used again to further train the fast R-CNN. As a result, both the RPN and the fast R-CNN use the shared convolutional layers to form a unified network as a faster R-CNN [72].

\subsubsection{Abnormality Detection with YOLO V2}

The R-CNN type of approaches belong to two-stage DL object detectors. Besides them, one stage DL detectors include You Only Look Once (YOLO) [103], YOLO V2 [104] and Single Shot MultiBox Detector (SSD) [100]. Instead of using cascaded steps for region proposal prediction and object detection, these models use one single network for the task.

- YOLO - It frames object detection as a regression problem to spatially separated bounding boxes and associated class probabilities. It uses a single neural network, which predicts bounding boxes and class probabilities directly from full images in one evaluation. It can be optimized end-to-end on detection performance.

- YOLO v2 - It makes various improvements to the YOLO detection method to make it better and faster. The improvements include batch normalization, pretraining a high-resolution classifier, predicting offsets rather than coordinates, computing dimension clusters, multi-scale training, etc. YOLO 9000 further improves the YOLO v2 model to be stronger through a joint training algorithm to train a model on more than 9,000 classes from ImageNet as well as detection data from COCO.

- SSD - It discretizes the output space of bounding boxes into a set of default boxes over different aspect ratios and scales per feature map location. At prediction time, the network generates scores for the presence of each object category 
in each default box and produces adjustments to the box to better match the object shape. In addition, the network combines predictions from multiple feature maps with different resolutions to naturally handle objects of different sizes.

According to [104], YOLO v2 achieved the highest mean average precision (mAP) of 78.6 on COCO data set, in comparison to 76.8 achieved by SSD and 76.4 by faster R-CNN. Because of the performance difference, we also train YOLO v2 as the abnormality detector for comparison on the mammogram data set.

For a limited data set, transfer learning has proved to be effective in reusing deep learning models pre-trained on large-scale data sets. The idea of transfer learning for classification also applies to object detection. The deep object detectors rely on convolutional layers for feature computation and those layers can be directly transferred from existing deep CNNs that have been trained on large-scale data sets such as the ImageNet. This can effectively reduce the amount of training data that are needed to train a DL-based medical abnormality detector.

\subsection{Results}

\subsubsection{Abnormality Detection with Class Activation Mapping Data Selection}

In mammography, there is a lack of standard evaluation data and most CADe algorithms are evaluated on private data-sets. This poses a challenge for comparing performance of methods or for replicating prior results. The most commonly used databases are the Mammographic Image Analysis Society (MIAS) database [105] and the Digital Database for Screening Mammography (DDSM) [106]. MIAS contains left and right breast images for 161 patients. There are 208 normal, 63 benign and 51 malignant images. It also includes radiologist's 'truth'-markings on the locations of 
any abnormalities that may be present. DDSM is the largest mammography data-set that is publicly available. The database contains approximately 2,500 studies, each includes two images of each breast, along with associated patient information and image information. Images containing suspicious areas have associated pixel-level "ground truth" about the locations and types of suspicious regions.

Recently, Lee et al. [78] released an updated and standardized version of the DDSM for the evaluation of CADe systems in mammography. Their data-set, named CBISDDMS (Curated Breast Imaging Subset of DDSM), includes decompressed images, data selection and curation by trained mammographers, updated mass segmentation and bounding boxes, and pathologic diagnosis for training data. The data-set contains 753 calcification cases and 891 mass cases. Sample image patches are shown at the top row of Figure 16.

We use image patches from CBIS-DDSM for classification and use full mammograms for abnormality detection test. We merge the training and testing data-set in CBIS-DDSM and conduct new 85/15 split for training and testing sets. The number of image patches are listed in Table 6.

Table 6: Size of training and testing image patches for CBIS-DDSM mammogram data-set.

\begin{tabular}{|l||c|c|c|}
\hline Abnormality & Training & Testing & Overall \\
\hline \hline Calcification & 1284 & 227 & 1511 \\
Mass & 1353 & 239 & 1592 \\
\hline
\end{tabular}




\section{Data Augmentation}

To avoid over-fitting, we applied data augmentation on the training data-set: random rotations between zero and 360 degrees, and random $\mathrm{X}$-axis and $\mathrm{Y}$-axis reflections. This procedure is motivated by our observation of the variations within the data-set.

\section{Comparison of Different Deep CNN Architectures}

We set the following parameters for training a deep patch CNN: Stochastic Gradient Descent with Momentum (SGDM) as the optimization algorithm, batch size of 16, initial learning rate as $1 e-4$, and the learning rate factor for the last fully connected layer as 20.0. Each network stops from further training if the mean accuracy on the fifty most recent batches reaches $99.5 \%$ or if the number of epochs reaches 200 . All the models are trained on a workstation with an NVIDIA GeForce GTX TITAN X GPU (one hour for AlexNet, eight hours for VGGNet, two hours for GoogLeNet, and four hours for ResNet). The final size of fine-tuned VGGNet is about 20 times that of GoogLeNet, with in-between sizes for AlexNet and ResNet.

Final results are listed in Table 7, after running cross validations and computing mean accuracy across the five folds. VGGNet achieves the highest accuracy for classi-

fying calcification and GoogleNet gets the best performance on classifying mass. The highest overall accuracy is achieved by VGGNet.

In order to compare with traditional machine learning approaches, we select support vector machines (SVMs) as one of the representative classifiers. To conduct fair comparisons with the deep CNNs, we apply transfer learning using pre-trained deep CNNs as feature extractors. Without loss of generality, we use AlexNet that has been pre-trained on ImageNet data-set and compute features after each principal layer, i.e, convolution layer or fully connected layer. We then feed these features to an SVM model, train the classifier, and test their performance on the same testing data as 
Table 7: Mean Classification Accuracy of Deep CNNs and SVMs on the Mammogram Data-set

\begin{tabular}{|l||c|c|c|}
\hline Model & Calcification & Mass & Overall \\
\hline \hline AlexNet & $88.81 \%$ & $93.64 \%$ & $91.23 \%$ \\
VGGNet & $\mathbf{9 2 . 4 2} \%$ & $92.64 \%$ & $\mathbf{9 2 . 5 3} \%$ \\
GoogleNet & $87.14 \%$ & $\mathbf{9 5 . 0 6} \%$ & $91.10 \%$ \\
ResNet & $90.22 \%$ & $93.39 \%$ & $91.80 \%$ \\
\hline AlexNet-conv5-SVM & $87.05 \%$ & $87.62 \%$ & $87.33 \%$ \\
AlexNet-conv5-100-ANN & $87.40 \%$ & $89.37 \%$ & $88.39 \%$ \\
HOG-SVM & $83.70 \%$ & $79.50 \%$ & $81.55 \%$ \\
\hline
\end{tabular}




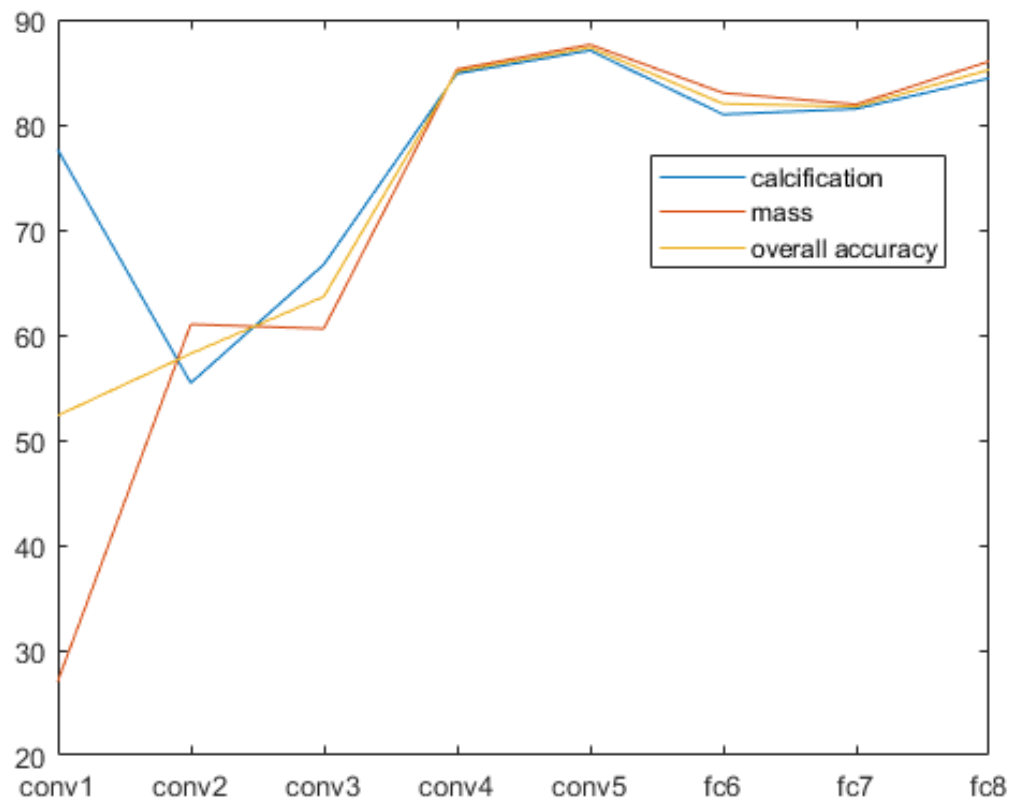

Figure 18: Accuracy of SVM models trained with features computed from the different layers in the AlexNet (mammogram data-set). 
those in previous experiments. The same five-fold cross validation is conducted and the result is plotted in Figure 18 over the different feature extraction layers. It indicates that the accuracy of the SVM model keeps increasing over the convolution layers until "conv5" and then drops at fully connected layers. The best result is included in Table 7 with the model named 'AlexNet-conv5-SVM'.

Besides SVM, we also implement transfer learning with artificial neural networks $(\mathrm{ANN})$ as the classifier. Varying the number of neurons in the hidden layer leads to different values of accuracy. After a series of experiments, the best performance is achieved with 100 neurons in the hidden layer. The result is included in Table 7 with the model named 'AlexNet-conv5-100-ANN'.

In comparison with manual features, a model trained with histogram of oriented gradients (HOG) using SVM ('HOG-SVM') achieved only $81.55 \%$ accuracy. HOG is one of the commonly used image features in computer vision [32]. These results indicate that the deep CNNs outperform the traditional methods on patch classifications of both calcification and mass, because of more effective features and betterperforming classifiers.

\section{Detection Results using Class Activation Mapping}

We choose fine-tuned ResNet for computing class activation mappings, based on the fact that ResNet is ready for use without further training. To test the CAM, we use one full mammogram image with calcification, feed it to ResNet, compute the CAM and show the result in Figure 19. The heat-map is superimposed on the mammogram image in the middle, which highlights the location of calcification found in the mammogram (best viewed in color). For comparison, we also include the ground-truth binary mask image on the right. 

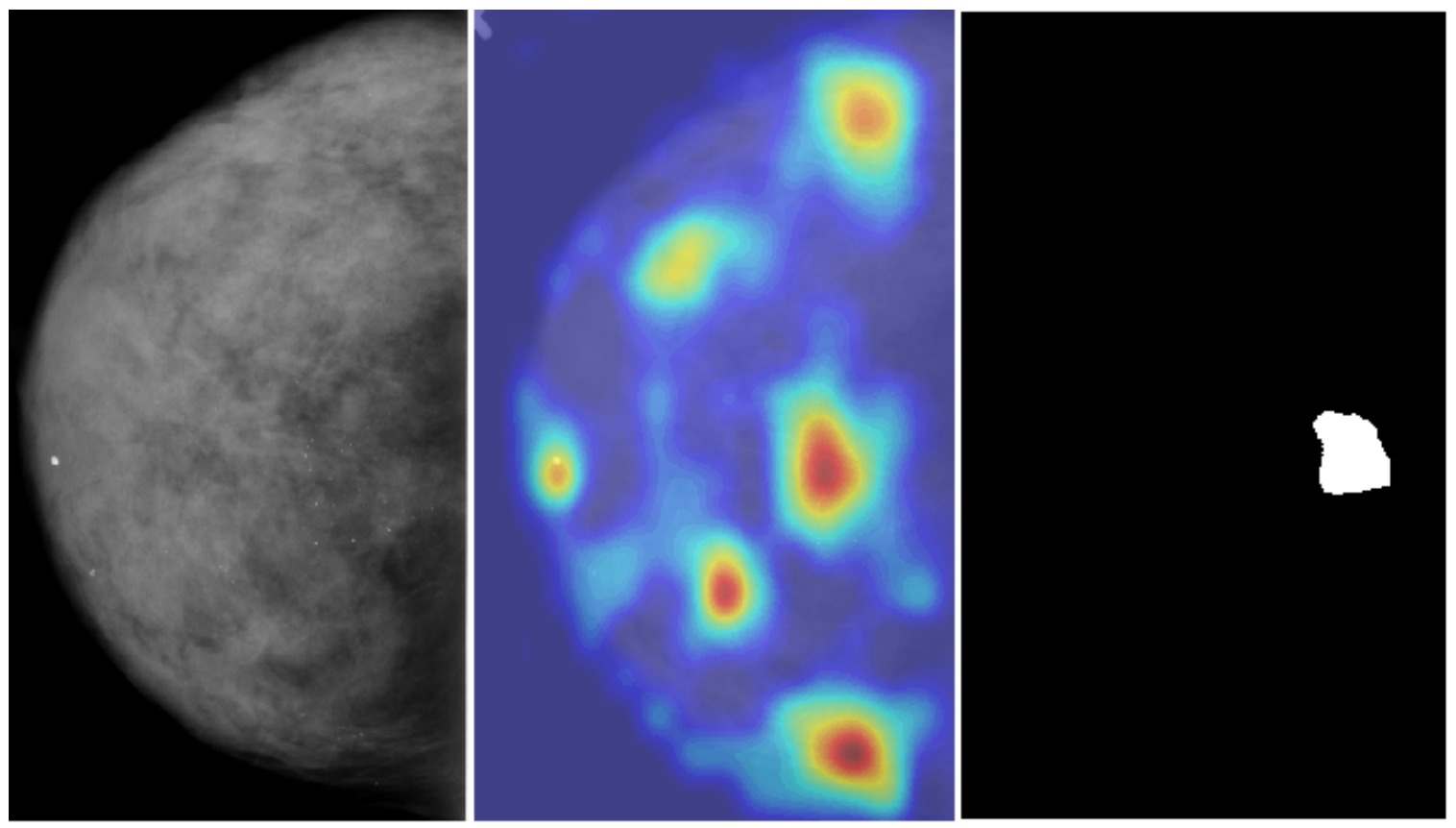

Figure 19: Calcification localization result (left: full mammogram, middle: class activation map output superimposed on the mammogram, right: ground-truth binary mask image). 
Similarly, a full mammogram from the mass class is fed into the ResNet for computing the CAM. The results are demonstrated in Figure 20. The highlighted heatmap matches well with the binary mask image on abnormality locations.
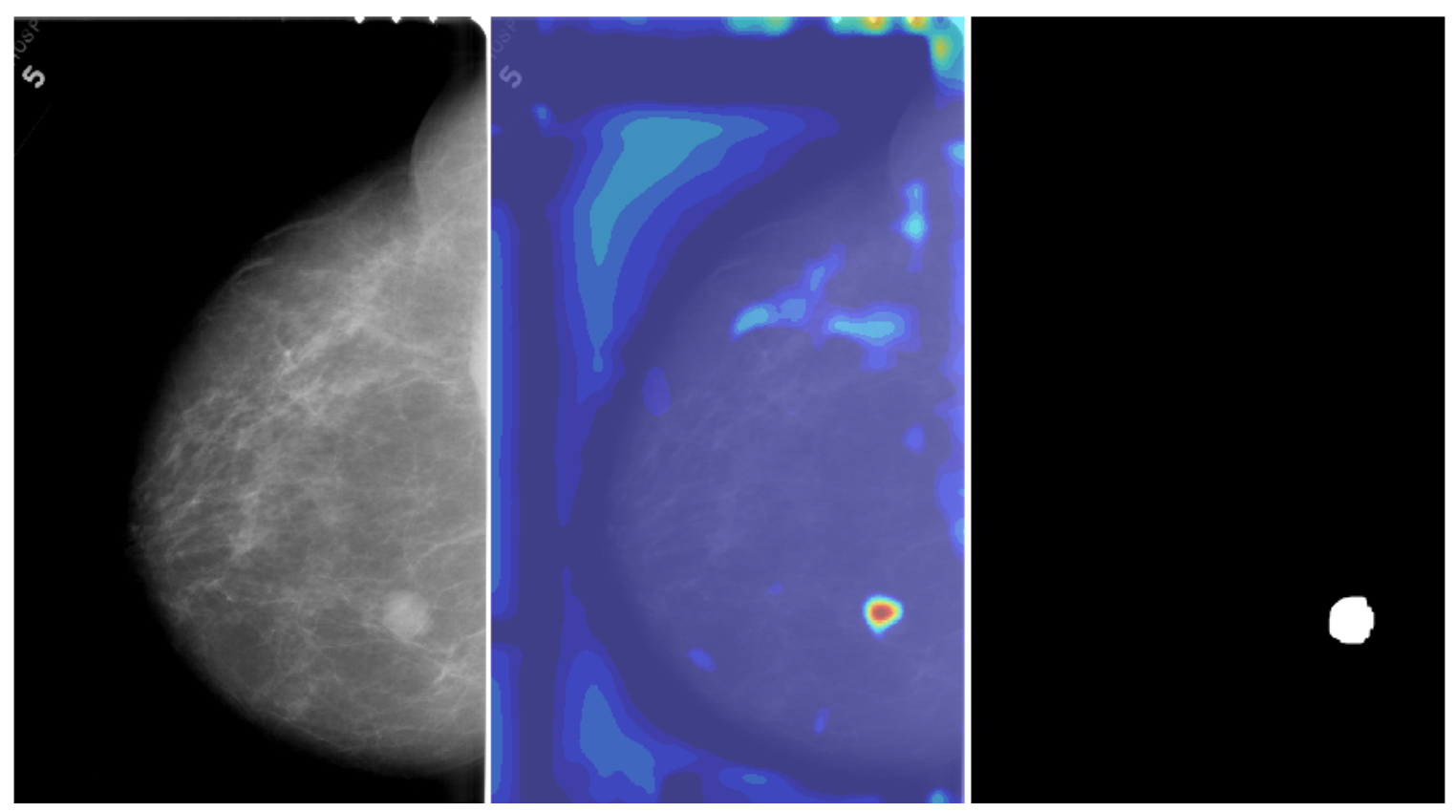

Figure 20: Mass localization result (from left to right: full mammogram, class activation map output superimposed on mammogram, ground-truth binary mask image).

To further evaluate the effectiveness of CAM, we have conducted experiments on more mammograms and placed the outputs in Figure 21 and 22.

\subsubsection{Abnormality Detection with Faster R-CNN}

\section{Data Selection}

Comparing with other modalities in medical imaging, ultrasound has advantages of being lower cost and more accessible; however, the image quality is much lower than that of CT or MRI images. Therefore, detecting abnormalities in ultrasound images is very challenging. In this work, we adopt a private ultrasound liver data-set from Nantong No. 3 People's Hospital (city of Nantong, China), termed Nantong data-set. 

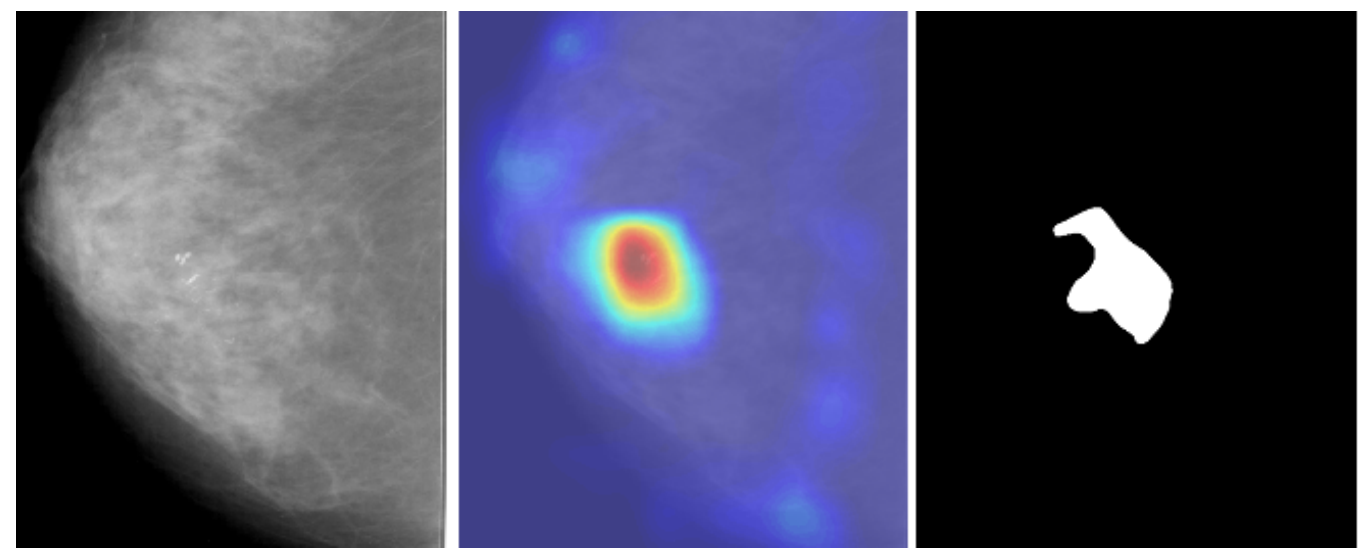

(a) Test case 1
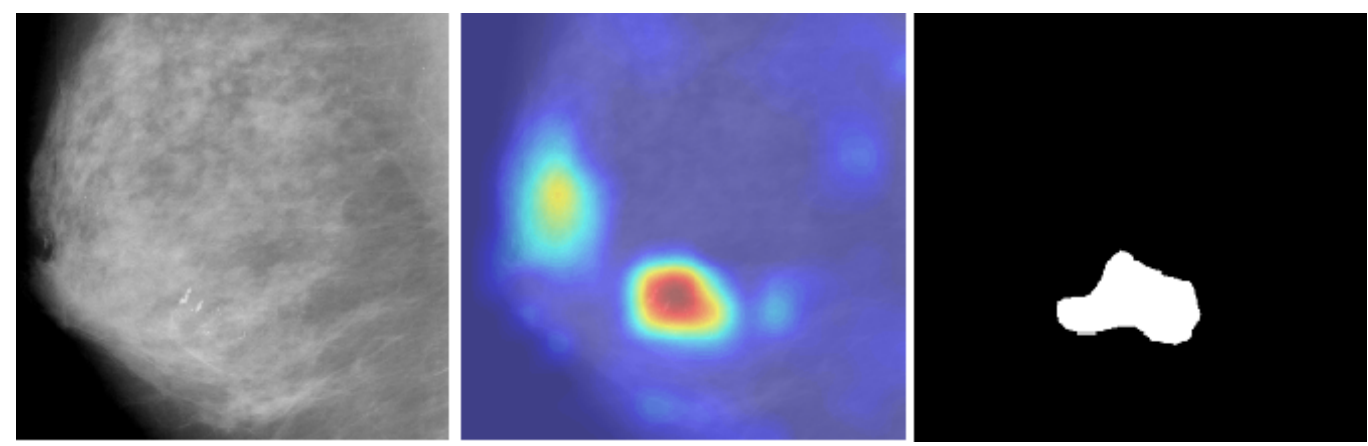

(b) Test case 2
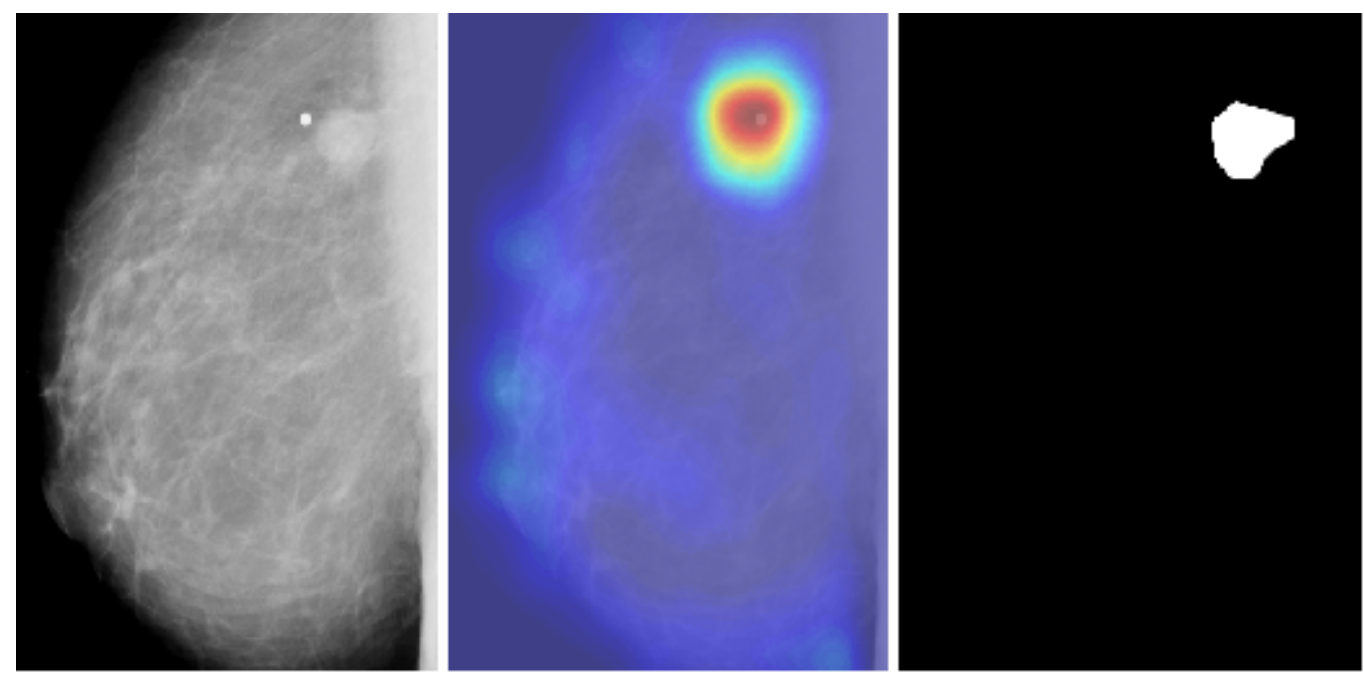

(c) Test case 3

Figure 21: Calcification localization result (left: full mammogram, middle: class activation map superimposed on mammogram, right: ground-truth image). 

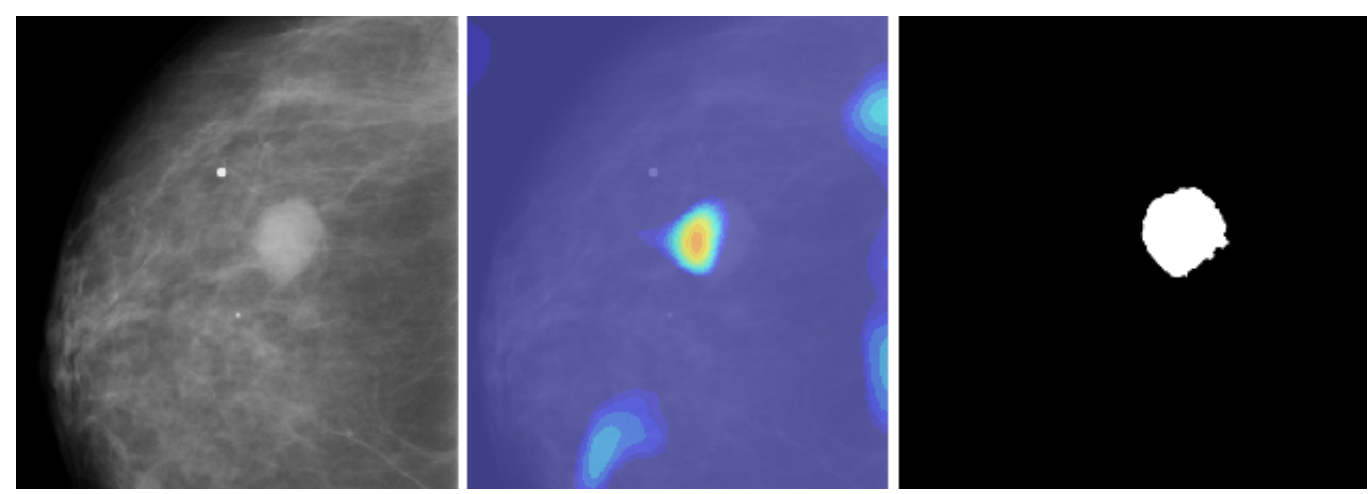

(a) Test case 4
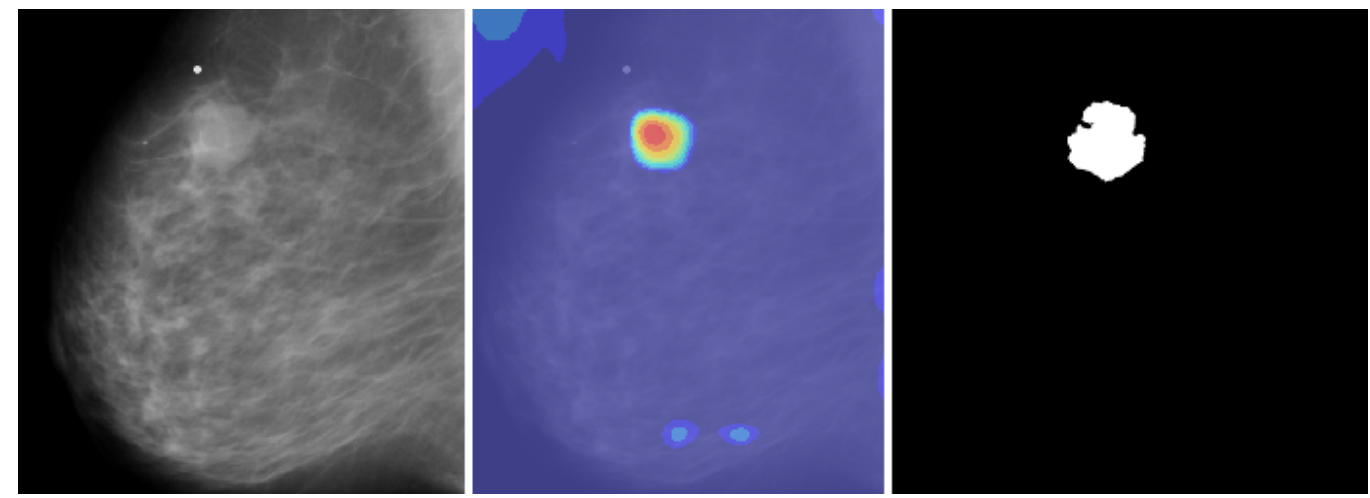

(b) Test case 5
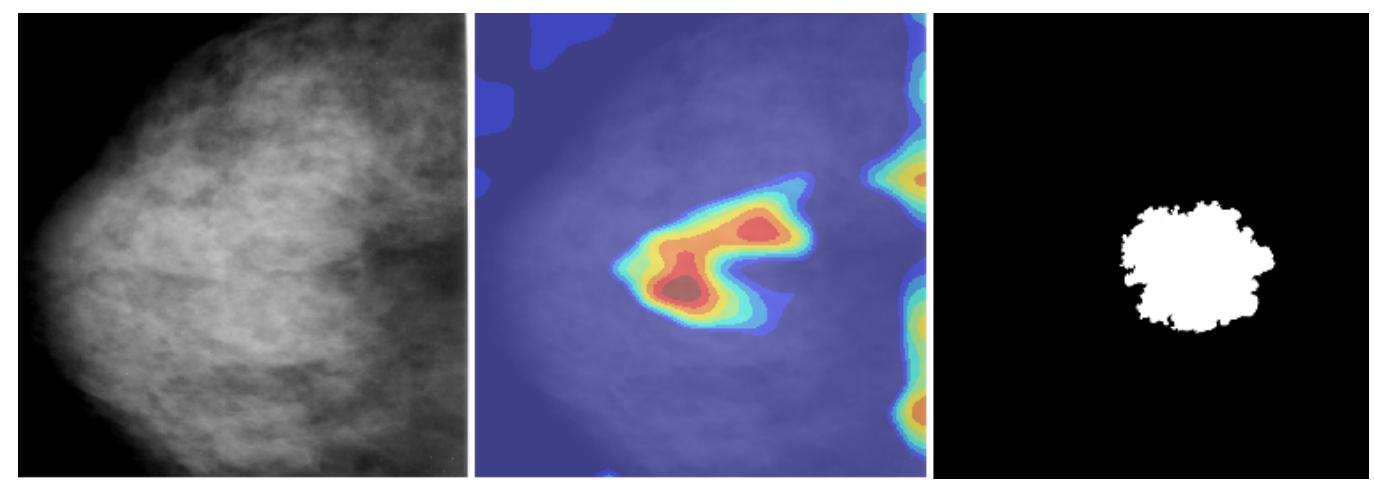

(c) Test case 6

Figure 22: Mass localization result (left: full mammogram, middle: class activation map superimposed on mammogram, right: ground-truth image). 
Patients' consent has been obtained on using their data for conducting research and publications. All the images have been sanitized to remove personal information. The number of images in each category are listed in Table 8. For each category, we conduct $80 / 20$ split for training and testing. Note that this is a much smaller data-set than the previous mammogram data-set.

Table 8: Size of training and testing sets from Nantong data-set of ultrasound liver images.

\begin{tabular}{|l||c|c|c|}
\hline Abnormality & Training & Testing & Overall \\
\hline \hline CYST & 231 & 58 & 289 \\
PHC & 83 & 21 & 104 \\
\hline
\end{tabular}

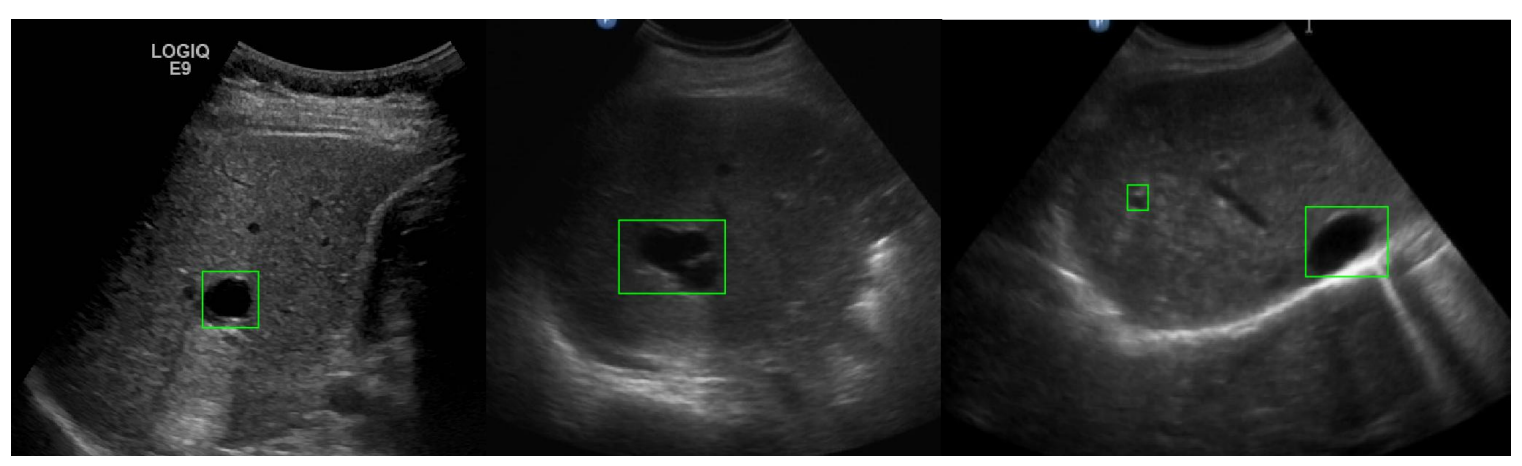

Figure 23: Sample images of the CYST data-set (labeled in green rectangles).

The two selected categories of abnormalities in US liver images are cyst and primary hepatic carcinoma (PHC). Sample US liver images with labeled cysts are illustrated with Figure 23 and sample PHC images are with Figure 24. 


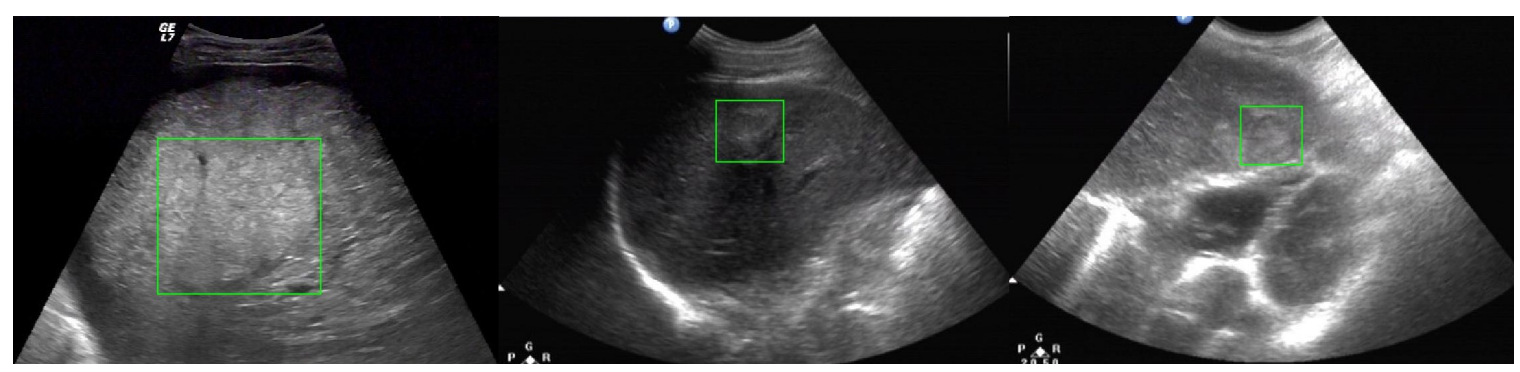

Figure 24: Sample images of the PHC data-set (labeled in green rectangles).

\section{Experiment Settings}

Fine-tuning a faster R-CNN detector on the Nantong data-set uses the following parameter settings. The four-state training process uses the stochastic gradient descent with momentum (SGDM) optimizer. Max epochs are set to 10 with mini batch size at 1 . Initial learning rates are set to $1 e-3$ for the first two stages and $1 e-4$ for the last two stages. For the detector, we set $[0,0.3]$ and $[0.6,1.0]$ for the negative and positive overlap ranges. Box pyramid scale is set to 1.2. Training each detector takes about half an hour on our GPU workstation.

The convolutional feature extractor in the faster R-CNN can be replaced with different state-of-the-art deep CNNs. Three of them are used for comparing the performance on the testing data. Their average detection precision is listed in Table 9. The average precision is computed for successful detection, in which the overlap ratio between the predicted bounding box and the ground-truth bounding box is above a threshold (we use 0.5 in our experiment). The results indicate that for CYST dataset, both GoogleNet and ResNet are showing the best and close performance among the three, while on the PHC data-set, ResNet is demonstrating the best precision.

In order to compare with traditional approaches, we train a SVM classifier using the HOG feature and apply it to testing images through a window-sliding approach (window size of $100 \times 100$ in pixels, step size of 20 pixels). To evaluate the traditional method, we use the first five highest-ranked bounding boxes among all the candidates 
from prediction. The average precision is computed and placed under the column of 'HOG-SVM' in Table 9.

Table 9: Average Precision of Deep Detectors on Different Abnormalities in the Ultrasound Liver Data-set

\begin{tabular}{|l||c|c|c|c|}
\hline Abnormalities & AlexNet & GoogleNet & ResNet & HOG-SVM \\
\hline \hline CYST & 0.41 & 0.57 & $\mathbf{0 . 6 0}$ & 0.16 \\
PHC & 0.15 & 0.26 & $\mathbf{0 . 4 2}$ & 0.18 \\
\hline
\end{tabular}

\subsubsection{Abnormality Detection with YOLO V2}

In order to compare with other deep learning approaches, we conduct a comparison with YOLO v2 model [104]. In general object detection, there are two main-stream types of deep learning approaches: two-stage and one-stage. The faster R-CNN is a two-stage network, which trains a region proposal network and an object detector in separate steps. YOLO v2 model belongs to the one-stage approach, in which the model trains one single network for object detection.

We train a series of YOLO v2 models at $448 \times 448$ resolution, using the Resnet50 network. The abnormality detectors are trained for CYST and PHC separately. For each abnormality, different learning rates are used for comparison. The average precision results are listed in Table 10. Layer "activation_40_relu" is used during training for activation. Minimum batch size is set at 16 and the number of epochs are set at 100. The anchor box sizes for PHC and CYST are [116 116, 169 155, 81 $75,5456]$ and $[5554,3538,8074,131$ 121].

The YOLO v2 models result in lower precision than those achieved by our proposed 
Table 10: Average Precision of YOLO v2 based Detectors in the Ultrasound Liver Data Set

\begin{tabular}{|l||c|c|c|}
\hline Learning Rate & $1.0 \mathrm{e}-5$ & $5.0 \mathrm{e}-5$ & $1.0 \mathrm{e}-4$ \\
\hline \hline CYST & 0.33 & 0.45 & $\mathbf{0 . 5 1}$ \\
PHC & 0.05 & $\mathbf{0 . 0 8}$ & 0.06 \\
\hline
\end{tabular}

approach. In the case of CYST, keep increasing the learning rate leads to a sudden drop of the precision towards zero. For PHC, the highest average precision by YOLO v2 model is much lower than that achieved by our approach.

A comparison on tables 9 and 10 indicates that, on the ultrasound image data set, Faster R-CNN model outperforms the traditional HOG feature, while YOLO v2 model outperforms the HOG only on the detection of CYST but not PHC. The lack of training data leads to a sudden drop of performance for YOLO v2 model in the detection of PHC. In order to verify this, we conduct similar comparisons on the mammogram data set where we have more training data. The results are listed in Table 11. It confirms that when more training data are available, the YOLO v2 model outperforms the HOG-SVM approach by a large margin and the Faster R-CNN approach still achieves the top performance among the approaches.

Among the selected deep CNN architectures (ResNet50, GoogLeNet and MobileNet v2), Faster R-CNN achieved the highest average precision using GoogLeNet and YOLO v2 used ResNet50 to achieve the best result. Details about this comparison, including experiment settings, are available in [19]. 
Table 11: Average Precision of Object Detectors on Mass Detection in the Mammogram Data-set

\begin{tabular}{|l||c|c|c|}
\hline Abnormality & HOG-SVM & Faster R-CNN & YOLO v2 \\
\hline \hline Mass & 0.0416 & $\mathbf{0 . 3 6 0 7}$ & 0.3107 \\
\hline
\end{tabular}

\section{Comparison with Traditional Approaches on Patch Classification}

To compare the deep CNNs with traditional approaches on classifications, we conduct feature transfer with SVM and ANN in a similar way as we did with the mammogram data-set. After five-fold cross validations, the results on patch classifications are listed in Table 12. Figure 25 depicts the performance on extracting features from different layers in AlexNet for training the SVM classifier (layer 'conv5' leads to the best result and thus listed as 'AlexNet-conv5-SVM' in Table 12). In training an ANN model, our experiments find that a number of 60 neurons in the hidden layer gives the best classification result (listed as 'AlexNet-conv5-60-ANN' in Table 12). When not using the deep CNN features, a model trained with HOG features using SVM ('HOG-SVM') achieved only $87.29 \%$ accuracy. The results indicate that the deep CNNs outperform the traditional methods on patch classifications of both cyst and PHC, because of more effective features and better-performing classifiers.

\section{Detection Results}

We now make observations on the detection results. Figure 26 demonstrates the detection results on two test cases. The first test case is examined with the trained CYST detector based on different deep neural networks (AlexNet, GoogleNet and ResNet). Likewise, the second test case is examined with the trained PHC detector 
Table 12: Mean Accuracy of Deep CNNs and SVMs on the Liver Data-set

\begin{tabular}{|l||c|c|c|}
\hline Model & CYST & PHC & Overall \\
\hline \hline AlexNet & $94.88 \%$ & $95.00 \%$ & $94.94 \%$ \\
GoogleNet & $\mathbf{9 9 . 5 3} \%$ & $88.75 \%$ & $94.14 \%$ \\
\hline ResNet & $98.60 \%$ & $\mathbf{9 8 . 7 5 \%}$ & $\mathbf{9 8 . 6 8} \%$ \\
\hline AlexNet-conv5-SVM & $96.28 \%$ & $95.00 \%$ & $95.64 \%$ \\
HOG-SVM & $95.81 \%$ & $95.00 \%$ & $95.41 \%$ \\
\hline
\end{tabular}

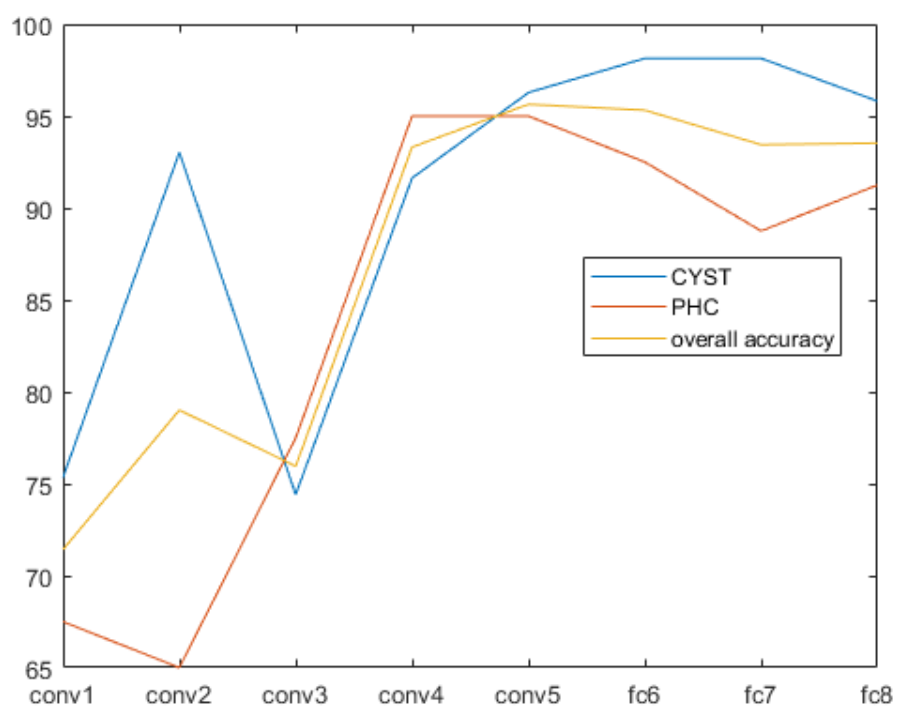

Figure 25: Accuracy of SVM models trained with features computed from the different layers in the AlexNet (liver data-set). 
based on the different deep network architectures. In most cases, the detectors show good performance in that the detected areas have large overlapping with the groundtruth area. The exception is with case 2 using a GoogleNet based PHC detector, where the predicted area includes but is larger than the ground-truth.

We conduct further experiments on other testing cases. Figure 27 demonstrates the detection results with the CYST detectors on two new testing cases. In (a) and (b), the CYST detectors successfully identified the CYST labeled by doctors. However, both detectors also detected one more area as CYST located lowed in the image. The radiologist confirms that this is an inferior vena cava (or IVC), which is a large vein that carries de-oxygenated blood from the lower and middle body into the right atrium of the heart. In (c), the CYST detector detects one more area as CYST with a probability at 0.91218. The radiologist confirms that this is actually a portal vein, which is a blood vessel that carries blood from the gastrointestinal tract, gallbladder, pancreas and spleen to the liver. Therefore the IVC and portal vein are detected as false positives by the detectors.

In another test case (case 4), the detectors are successful in detecting the CYST previously labeled by the radiologist. Without exception, the three detections also locate a new area as CYST (upper left in the image) with different but high probabilities. The radiologist confirms that the newly located area is actually a CYST, which was neglected during the labeling process.

In summary, the detectors are successful in locating the actual CYSTs in the testing images; however, they make false positive detection in certain cases but are able to identify CYSTs that may have been missed by human annotators.

Furthermore, we conduct more experiments on testing cases in the HPC category. Figure 28 demonstrates the detection results on two new cases. For case 5, the three detectors are able to detect the PHC close to ground truth but at different probabilities. It is noted that AlexNet detects a separate area as PHC, located left 


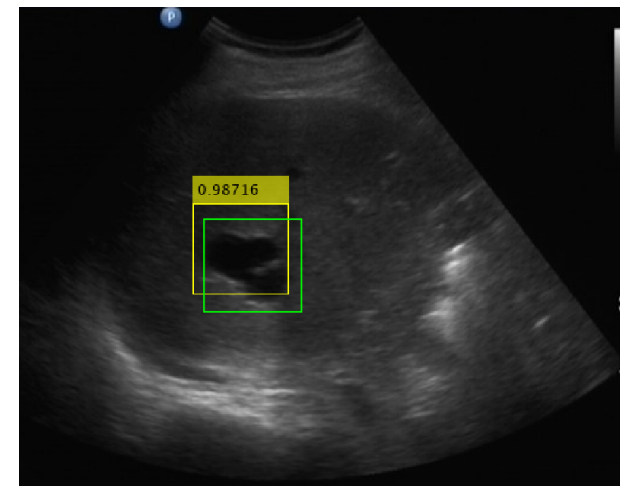

(a) Case 1, AlexNet

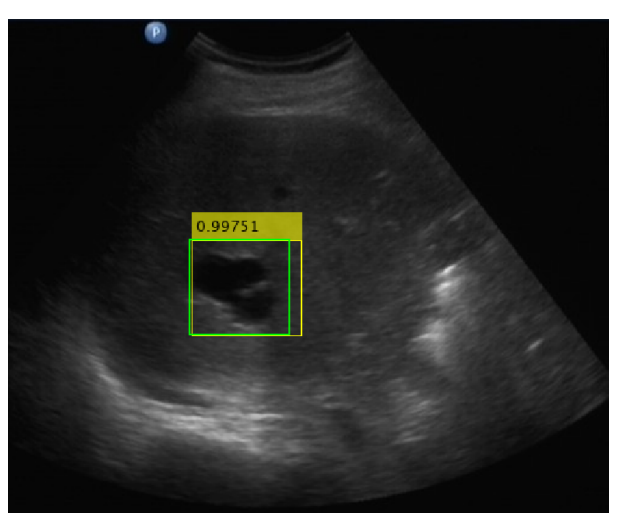

(c) Case 1, ResNet

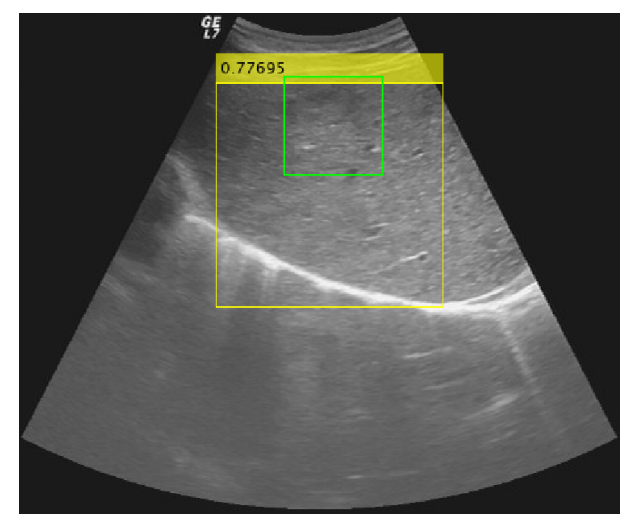

(e) Case 2, GoogleNet

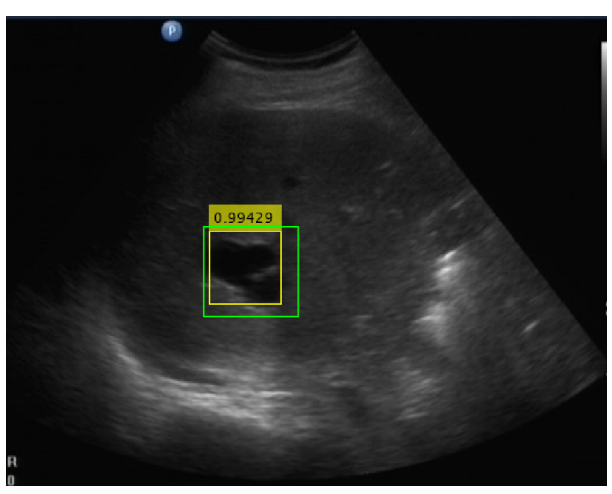

(b) Case 1, GoogleNet

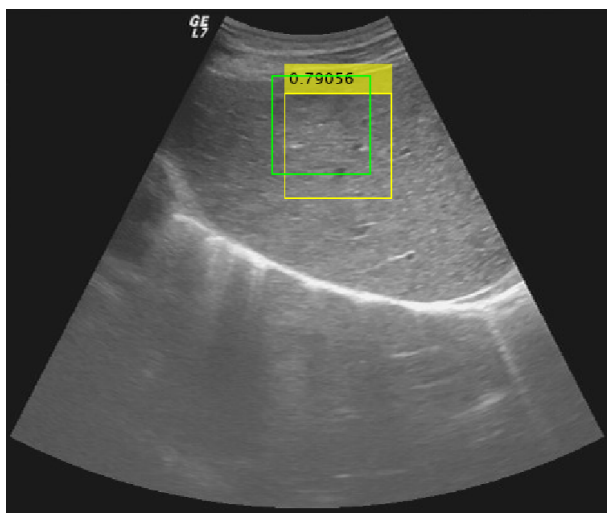

(d) Case 2, AlexNet

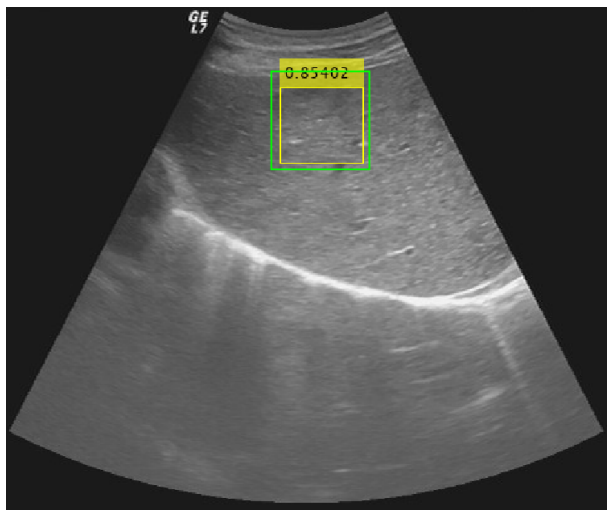

(f) Case 2, ResNet

Figure 26: Detection results on two test cases (case 1: (a) - (c) with CYST abnormality; case 2: (d) - (f) with PHC abnormality). Detection results are labeled in yellow with probabilities and ground truth is labeled with green rectangles. 


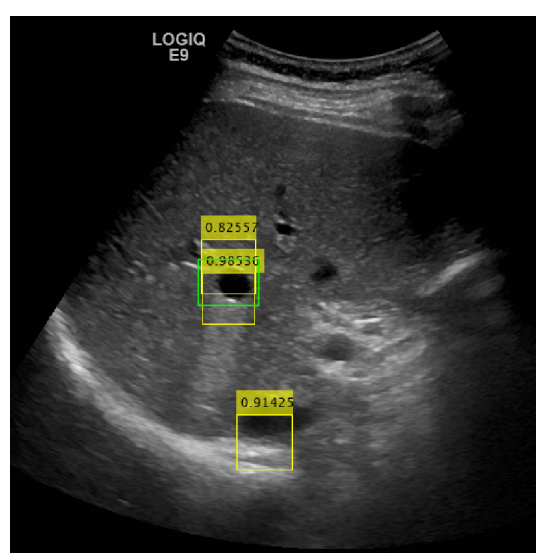

(a) Case 3, AlexNet

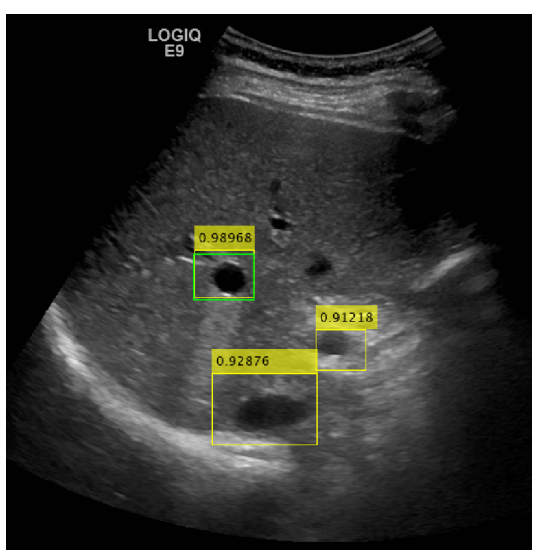

(c) Case 3, ResNet

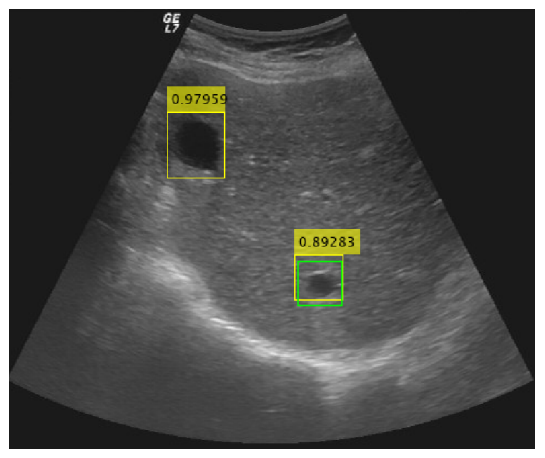

(e) Case 4, GoogleNet

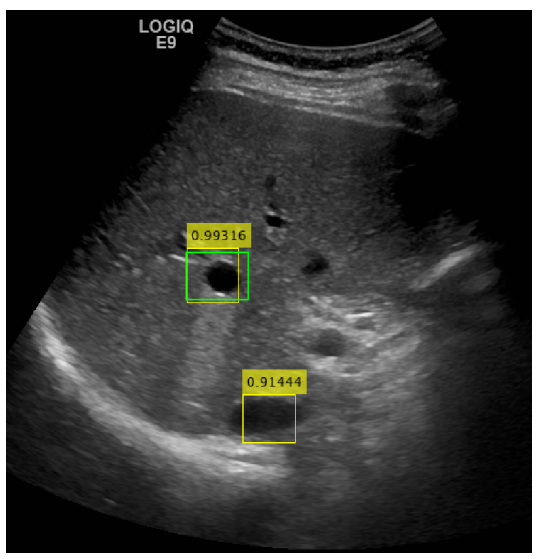

(b) Case 3, GoogleNet

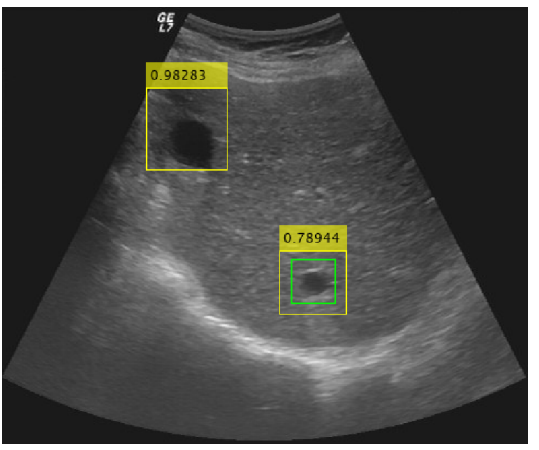

(d) Case 4, AlexNet

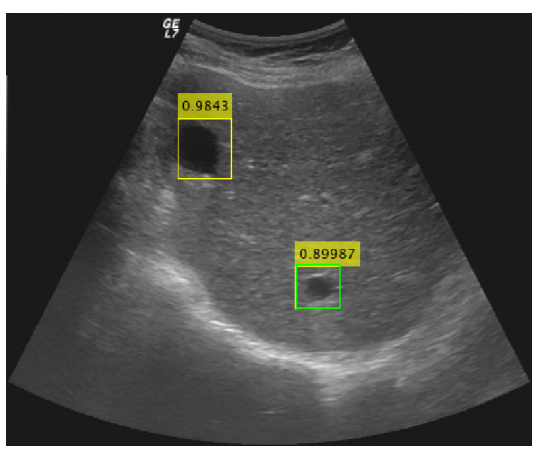

(f) Case 4, ResNet

Figure 27: Detection results on two test cases with CYST abnormality (detection results are labelled in yellow with probabilities, ground truth is labelled with green rectangles. 
to the ground-truth. The radiologist confirms the correctness of this extra detection and points out that this belongs to metastatic or invasive lesion, which is an area that should have been labeled by human annotators.

Case 6 is arguably the most challenging one among all the testing cases in demonstration. Patient record confirms that the patient had a history of cirrhosis, which is a late stage of scarring (fibrosis) of the liver caused by many forms of liver diseases and conditions, such as hepatitis and chronic alcoholism. Empirically, the ResNet-based detector is the most successful in detecting the PHC area (see (f) in the figure). In comparison, the GoogleNet-based detector missed the PHC (see (e) in the figure). The interesting result comes from AlexNet-based detector ((d) in the figure), which fails to detect the annotated PHC but detect the other two areas as PHC without high probabilities. We present the result to the radiologist, who diagnoses this case as massive liver cancer (with diameter greater than 10 centimeters). Many nodules within the liver are dead and hard to find because of unclear boundaries. There is also no obvious presence of isolated liver mass which presses against periphyseal edema zone. This has caused the failure of GoogleNet-based detector in detecting PHCs. For this case, the experimental results indicate that combination of AlexNet-based and ResNet-based detectors gives the best result. The GoogleNet-based detector missed the diffusion lesions.

\subsection{Discussion}

It is challenging to train classifiers for detecting calcification and mass in mammogram images. Deep neural networks has a limitation on the size of input images $(224 \times 224$ or $227 \times 227$ in pixels). Resizing mammogram images to these sizes inevitably reduces their quality and lose the subtle details needed for classification. Therefore we propose training classifiers from cropped patch images in order to recognize the difference 


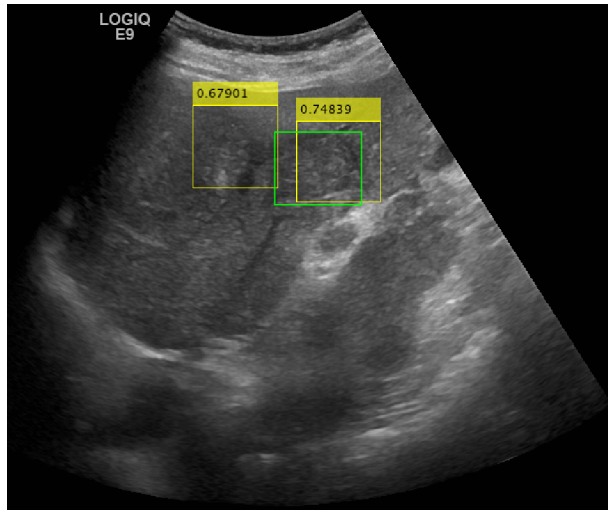

(a) Case 5, AlexNet

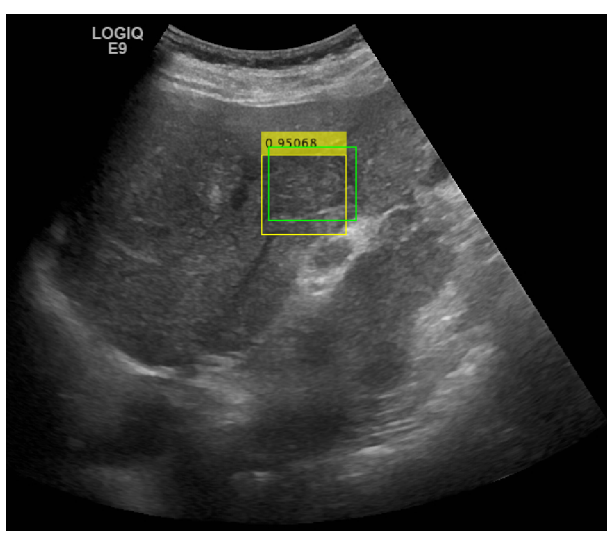

(c) Case 5, ResNet

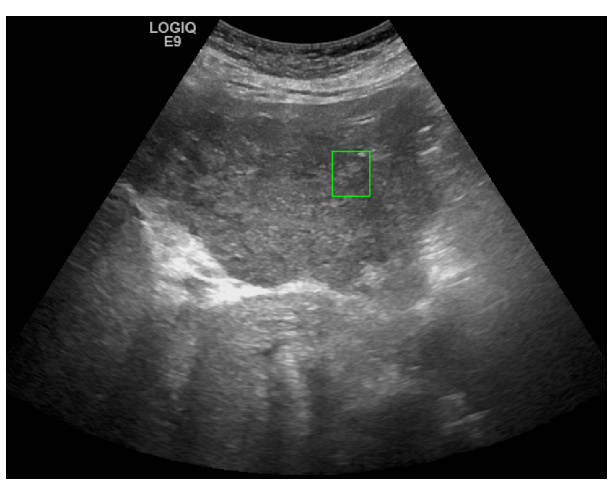

(e) Case 6, GoogleNet

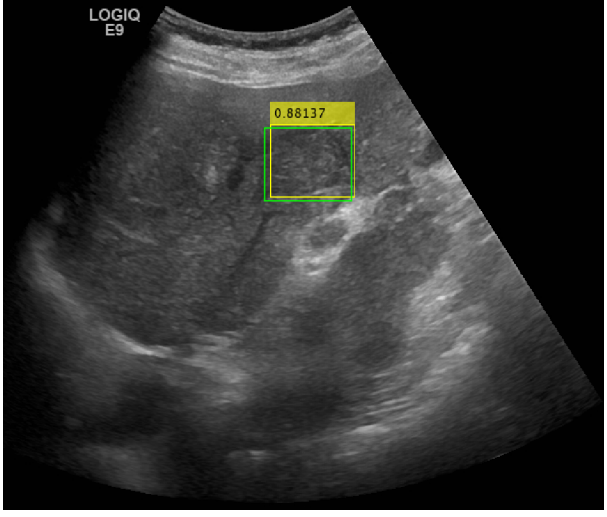

(b) Case 5, GoogleNet

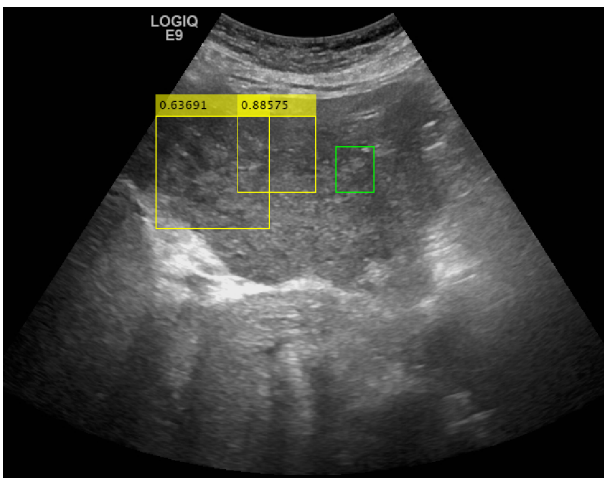

(d) Case 6, AlexNet

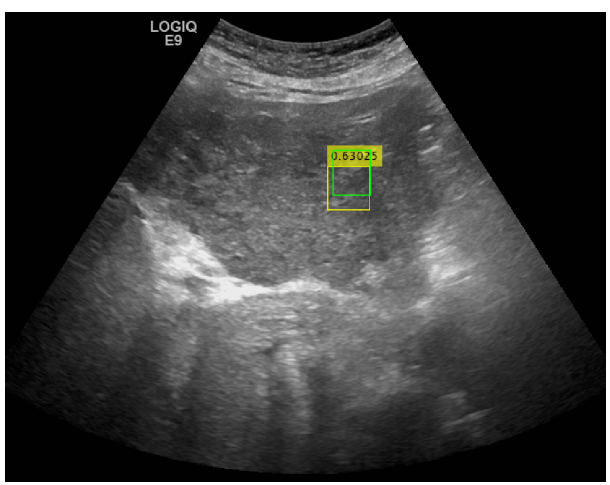

(f) Case 6, ResNet

Figure 28: Detection results on two test cases with PHC abnormality (detection results are labelled in yellow with probabilities, ground truth is labelled with green rectangles. 
between calcification and mass, and apply the trained deep CNN models onto fullsize mammogram images. Using a technique called class activation mapping, we successfully adapt the patch classifier for the localization of abnormalities in full mammogram images.

Detecting abnormalities in ultrasound liver images is more challenging because of low contrast and high level of noise. In most cases, the three detectors based on state-of-the-art neural networks achieve great success in detecting the abnormalities (CYST and PHC). For false positive cases, a cross-sectional view of the abnormality will help improving the detection result. The researchers and radiologist are pleased to see that the detectors are able to detect abnormalities that have been missed by human annotators. In very challenging cases, the combination of several detectors results in a more accurate and complete detection.

The CAM is easier for implementation while the faster R-CNN approach provides more information and options. The CAM approach relies on patch classifiers without needing to know where the patches are located in a full-size training image. Moreover, the CAM approach enables localizing abnormalities in one single forward pass, meaning that this method is more efficient than traditional sliding-window based approaches. Selecting different deep architectures for training faster R-CNNs leads to detectors of various performance. Combining the detectors is very effective for very challenging cases. A further combination with the CAM approach will help radiologists and doctors to avoid missing detection, and consequently improve patient health outcome.

In our recent research [20], we integrated the CAM approach with other deep detector models for lesion detection in MRI images. Bounding boxes were generated out of the CAM output and quantitative performance was then evaluated and compared. 


\subsection{Conclusion}

In this chapter, we validate our deep learning framework on high-resolution medical images, where abnormalities are localized in small areas and are hard to detect if images are compressed. We successfully integrate deep patch CNNs with class activation mappings and faster R-CNNs for medical abnormality detection. Because of limited training data, transfer learning is adopted for effective feature learning without over-fitting. Deep patch CNNs are trained to learn image features from local abnormalities. They are integrated with CAMs and RPNs for building abnormality detectors. Experimental results indicate that the integrated approach outperforms both traditional and other deep learning methods.

This chapter presents a branching method for abnormality detection under different training scenarios. Our recent research indicates that an integrated approach leads to better performance [20]. 


\section{Chapter 5}

\section{Representation Learning on 3D Data for Computing Features}

3D data are used for a large number of applications in medical imaging. Examples include scanning torso shape for scoliosis evaluation, scanning teeth shape for orthodontics, and scanning bone shape for surgical planning. Therefore, this chapter introduces the proposed framework for 3D human surface data, in order to compute viewpoint-invariant features for recognizing humans. In the proposed deep learning framework, components used in the validation are highlighted in Figure 29.

We study the viewpoint-independence of image features in the classification of identities using multiple-view full-body images. A reliable vision system should be robust in classifying objects from images captured on novel viewpoints. To obtain a robust classifier, 3D models are collected for rendering training and testing images from various viewpoints. These images are then used for extracting features and building classifiers. In this work, we compute multiple-view human-body images from a 3D anthropometry human body database. For each subject, a majority of the views are randomly selected to be included in the training dataset and the remaining views are used for testing. More specifically, we use Histogram of Oriented Gradient (HOG) feature based Support Vector Machine (SVM) as the baseline to be 

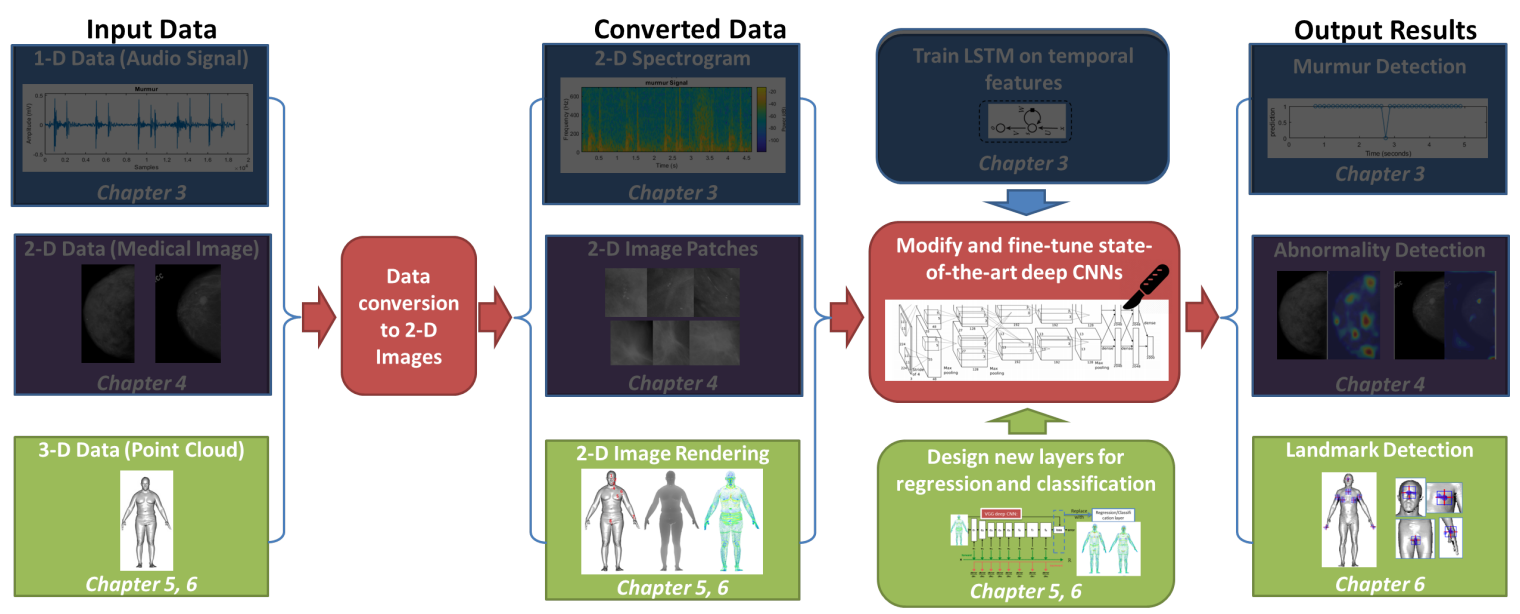

Figure 29: Applying the deep representation learning framework on 3D human surface data for human recognition (from left to right: raw data, converted 2D data representations, deep model training and output).

compared with deep auto-encoders network and deep Convolutional Neural Networks $(\mathrm{CNN})$. Through experiments, we conclude that the deep CNN performs the best (deep auto-encoders network as the runner-up) in computing viewpoint-independent image features for identity classifications based on 2D full-body images. For the deep CNNs, end-to-end training leads to better performance than using them solely as feature extractors.

\section{$5.1 \quad$ Introduction}

The study on viewpoint-independent image features remains an important research topic in computer vision. Image features with viewpoint independence play key roles in a wide variety of applications, ranging from classification, localization, recognition, tracking, to stereo matching.

Classifiers trained on certain views of multiple subjects may fail in recognizing 
images from novel viewpoints. For this challenge, 3D models have the advantage and capability of providing various views of the same object. In this work, we experiment on rendering multiple-view full-body images from a 3D anthropometry database and use the images for extracting features and building classifiers. We use a majority of the views for training and use the rest views for testing the identity classification rates.

Feature extraction plays a key role in building a robust classifier. In traditional machine learning, feature extraction is a time-consuming manual process. It normally involves image processing operations, such as computing image gradients to form discriminative feature descriptors for each image. The recent progress in deep learning enables automatic feature learning from images, eliminating the handdesigned/manual feature extraction step.

This chapter compares manual and automatic image feature extraction methods on viewpoint independence for identity classifications. More specifically, we compute, visualize, and compare image features using Histogram of Oriented Gradient (HOG) feature, deep network using auto-encoders, and deep Convolutional Neural Networks $(\mathrm{CNN})$. These features are then used to build classifiers for comparing identity classification rates on a testing dataset captured from different viewpoints.

To our best knowledge, this is the first work on applying deep neural networks onto the computation of view-independent image features on human bodies. Our contributions include: 1) visualizing image features and comparing their performance on identity classification; 2) comparing main-stream deep neural networks for automatic feature extractions; and 3) studying different structures of the deep neural networks for best performance. 


\subsection{Related Work}

Visual descriptors used for classification in computer vision can be divided into two categories: manual and automatic. The representative descriptors in the manual category include Histogram of Oriented Gradient (HOG) [32] and Local Binary Patterns (LBP) [30]. The automatic descriptors include those computed by deep neural networks (e.g., deep auto-encoders network and deep CNN).

In the original human detection task [32], C-HOG (Circular Histogram of Oriented Gradient) and R-HOG (Rectangular Histogram of Oriented Gradient) performed better on MIT and INRIA data sets [107] than the generalized Haar wavelets, PCA-SIFT (Principal Component Analysis - Scale Invariant Feature Transform [108]), and shape context descriptors [109]. By combining HOG with LBP, another work [110] has demonstrated improved performance on human detection.

Applying deep convolutional neural networks to 3D data has many challenges. One of them is the irregularity in data, which means variable size of unordered nodes and different number of neighbors. These challenges make convolutions difficult to apply. According to a recent survey paper on graph neural networks (GNN) [111], a graph convolution can be generalized from a 2D convolution through taking the weighted average of a node's neighborhood information. As one type of GNN, convolutional GNNs (ConvGNNs) can be used for node or graph classifications/regressions. The ConvGNNs have two main types: spectral-based approaches and spatial-based approaches. While the spectral-based approaches have a theoretical foundation in graph signal processing, spatial models have higher efficiency, better generality and flexibility [111]. In computer vision, the GNNs have applications in scene graph generation, point-cloud classification and action recognition. In particular for 3D meshes, geometric deep learning is more pertinent and promising [112]. 


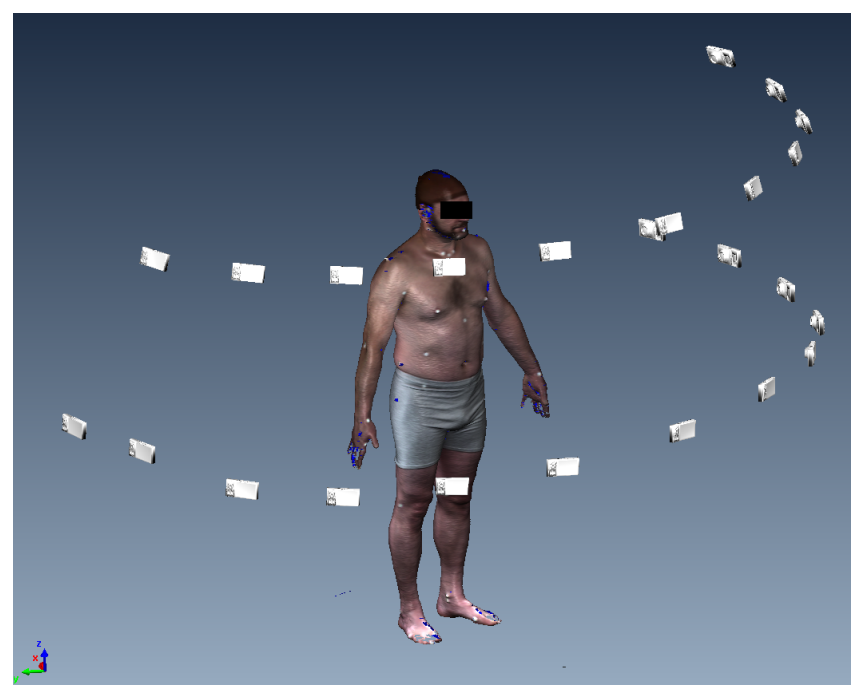

Figure 30: The setup of virtual cameras for capturing full-body images from different viewpoints

\subsection{The Approaches}

We introduce the 3D anthropometry dataset used in this chapter and the different feature extraction methods used for learning and building classifiers.

\subsubsection{Data Preparation}

We use the Civilian American and European Surface Anthropometry Resource (CAESAR) database for our experiments [113]. The CAESAR database is a large-scale 3D scan data set, which comprises full-body laser scans of civilian populations from North America, the Netherlands, and Italy. Besides 3D body scans, the data set also contains body measurements and demographic information, including age, gender, ethnicity, et al. The composition of the dataset can be found in Table 13 .

To get multiple-view full-body images, we use commercial software Polyworks to set multiple virtual cameras at different positions and angles (Figure 30). In 3D space, each virtual camera is set to face the $3 \mathrm{D}$ scan and the lighting is set to light up the 
Table 13: Composition of the CAESAR dataset.

\begin{tabular}{|c|c|c|c||c|}
\hline Region & North America & Italy & Netherlands & All \\
\hline Number of Subjects & 2384 & 800 & 1211 & 4395 \\
\hline
\end{tabular}
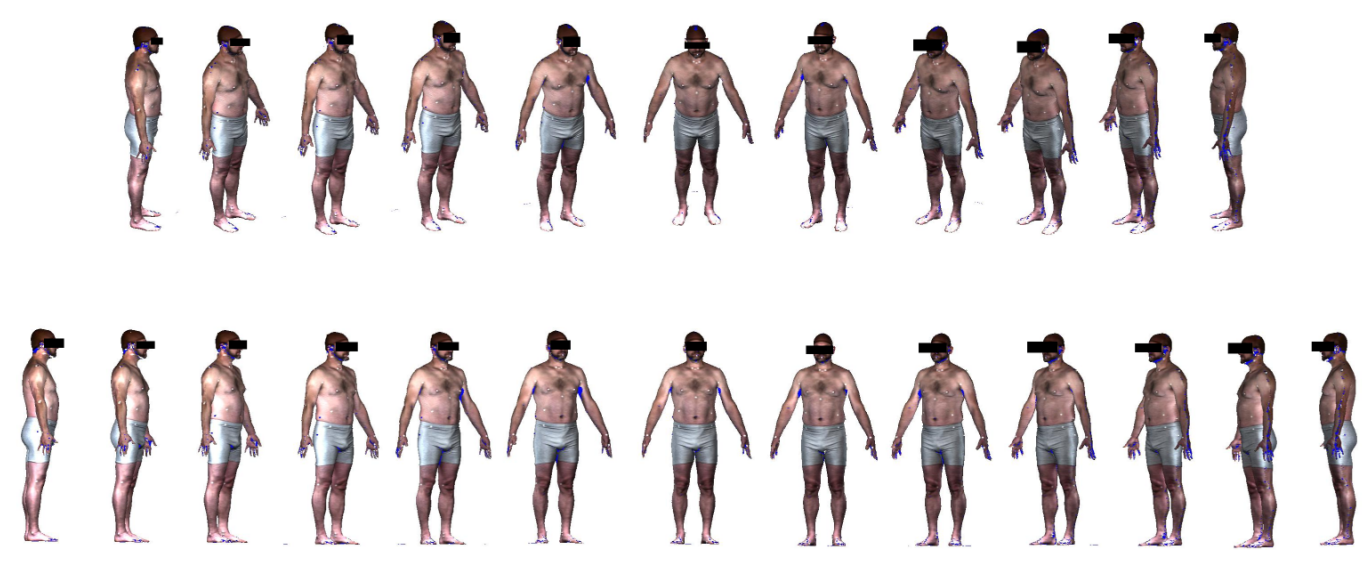

Figure 31: Multi-view images rendered from one 3D raw scan (top row: upper front views, bottom row: front views)

frontal surface of the 3D scan. Snapshots are then taken with the virtual cameras. Both the lighting and camera settings can be adjusted to achieve the best result on capturing full body images.

A data set of 2D full body images can be computed using the above setting. Our experiment uses 24 virtual cameras (11 upper front views and 13 waist-level front views). The rendered images of one subject are illustrated in Figure 31. All the faces are painted in black for removing the identity from the displayed subjects. 


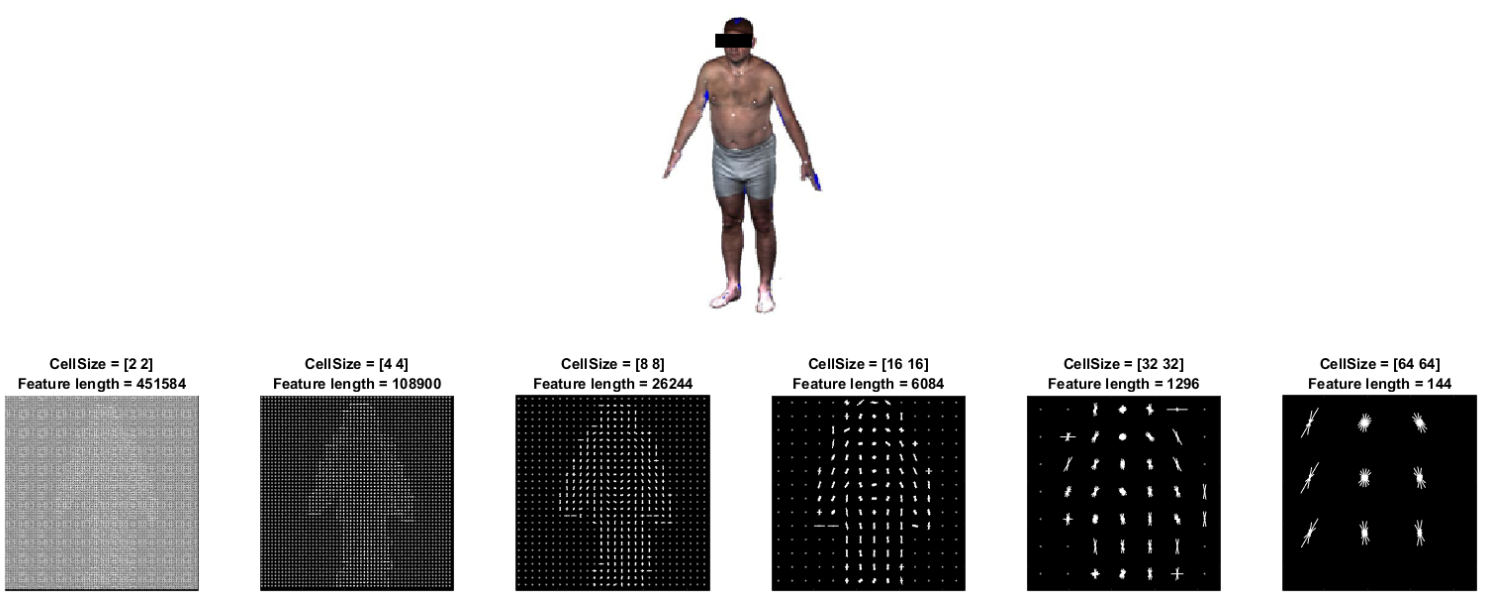

Figure 32: HOG features computed with different cell sizes on a sample image (top row: sample image, bottom row: computed HOG features (on top of each image icon are the cell size used for computing features and the length of the computed features))

\subsubsection{HOG Feature and SVM}

Histograms of Oriented Gradients (HOG) feature is selected for this study due to its simplicity of implementation and proved performance. The HOG feature is based on evaluating well-normalized local histograms of image gradient orientations in a dense grid [32]. It is based on the idea that local object appearance and shape can be characterized well by the distribution of local intensity gradients and edge directions. It can be done without knowing the corresponding gradient or edge positions.

To compute HOG features of an image, the image window needs first to be divided into small spatial regions called "cells". Each cell accumulates a local 1-D histogram of gradient directions or edge orientations over the pixels of the cell [32]. The combined histogram entries form the representation. Depending on the image dimension, cell size can be adjusted to compute the best features with a reasonable length of feature vector. Figure 32 demonstrates the HOG features computed on a sample image with different cell sizes. 


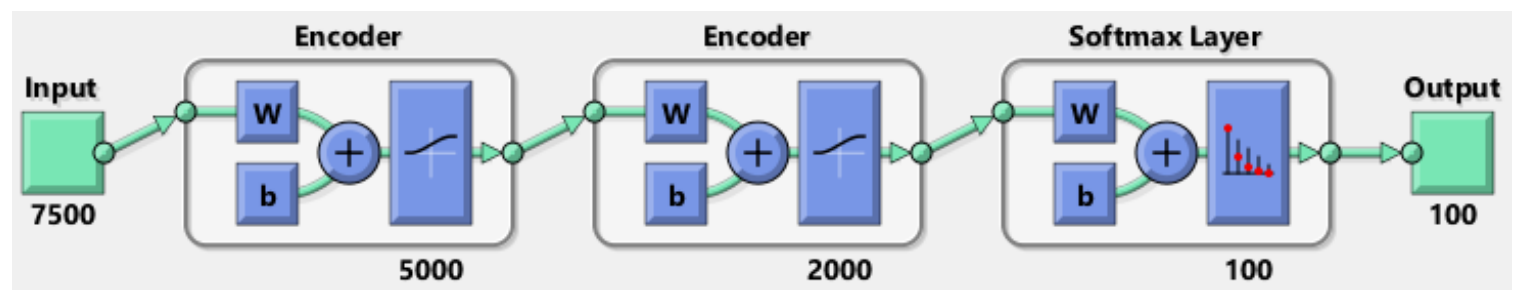

Figure 33: A deep neural network using auto-encoders

The computed HOG features are then fed into a linear Support Vector Machine (SVM) for training a classifier. Standard SVM supports two-class classifications. To build a multi-class classifier, we build multiple One-vs-One (OvO) SVM classifiers. Ultimately, $K(K-1) / 2$ classifiers are trained for a K-way multiclass problem [114]. At prediction, a voting scheme is applied to a new sample and the class with the highest number of votes from all the binary classifiers gets predicted by the combined classifier (multi-class SVM).

\subsubsection{Deep Auto-encoders Network}

Hinton et al. demonstrate in [34] that auto-encoders provide better performance in data compression than Principal Components Analysis (PCA). Auto-encoder trains a multi-layer neural network with a small center layer (the hidden layer) to reconstruct high-dimensional input vectors at output. Gradient-descent method is used to train the network.

An effective feature extractor can be built by stacking multiple encoders. A classifier can then be built by appending a softmax layer to the stacked encoders. Each encoder is trained in a unsupervised manner and the softmax layer is trained in a supervised manner. After assembling the encoders with the softmax layer, the whole neural network is again fine tuned in a supervised way. Figure 33 demonstrates a network composed of two encoders followed by a softmax layer. The numbers represent 
data dimensions in each layer.

\subsubsection{Deep Convolutional Neural Network and SVM}

The improvement on deep Convolutional Neural Networks (CNN) has brought tremendous progress in automatic feature learning $[6,8,9,115]$. In case of insufficient training data, learning transfer has proven to be effective.

Feature computation with convolutional layers. In our work, we apply the pre-trained AlexNet [6] to computing features for the multi-view full-body images, followed by a SVM classifier to be learned on the extracted features. A visualization of the first convolutional layer features is available in [6] ( [5] introduces details on visualizing features from other convolutional layers). The convolutional features vary depending on actual training images; however in general, the first convolutional layers represent "primitive" geometry features and the deeper layers form higher level image features.

The classifier. We build a multi-class SVM classifier based on the feature vectors computed from the deep convolutional neural network [6].

\subsubsection{End-to-End Training using Deep CNNs}

Besides using pre-trained deep CNNs as feature extractors, the state-of-the-art deep CNN models can be fine-tuned end-to-end on the target data set. Therefore, for a pretrained deep CNN, the output layers can be replaced with new ones that correspond to the number of output classes in this project. Higher learning rates are assigned to the newly replaced layers so that they can be actively trained on the target data set. In comparison, the other layers remain relatively unchanged during the fine-tuning process. 


\subsection{Experiments and Implementations}

\subsubsection{Datasets}

The dataset are selected to meet the requirements and limitations from all the approaches. The images of one hundred subjects are selected for identity classification. This essentially means a 100-class classifier needs to be built for each method. As we have 24 views rendered for each subject, together we have 2,400 images in the final dataset. For each class, 17 views are randomly selected to be included in the training dataset and the remaining 7 views are included in the testing dataset.

The AlexNet requires the input images to have a size of [227x227x3]. Therefore, all the training and testing images are scaled to this size before feature extraction and classifier building. The exception is for auto-encoders where the images are scaled to [50x50x3] because of the limited computer memory size.

All the experiments are conducted using Matlab (v 2016a) on an HP Z620 workstation (Intel Xeon CPU E5-1650 3.5 GHz, 64GB memory). All computations are made on CPU.

\subsubsection{HOG Feature and SVM}

We experimented on different cell sizes for computing the HOG feature. We then use the fitcecoc function in Matlab for training a multi-class SVM classifier. Classification rates for different cell sizes are $37.71 \%$ (32x32), 50.14\% (16x16), and $\mathbf{5 0 . 7 1 \%}(8 \times 8)$ respectively. It takes sixteen minutes to compute the HOG features and build an SVM classifier. 
Table 14: The comparison on classification rates for different dimensions of hidden layers in the auto-encoder networks. The last column lists the number of epochs when reaching minimum gradient during fine-tuning.

\begin{tabular}{|c|c|c|}
\hline Network Structure & Classification Rate & Number of Epochs \\
\hline $7500-1500-300$ & $62.71 \%$ & 746 \\
$7500-2500-800$ & $65.00 \%$ & 570 \\
$7500-5000-2000$ & $\mathbf{8 3 . 1 4 \%}$ & 773 \\
$7500-6000-5000$ & $81.29 \%$ & 551 \\
\hline
\end{tabular}

\subsubsection{Deep Auto-encoders Network}

As the auto-encoders are fully connected networks, the deep auto-encoders network consumes a great amount of computer memory if the input and hidden layers have large sizes. Due to this limitation, our experiments resize all the RGB images to a reduced dimension $(50 \times 50 \times 3=7,500)$. Different results are achieved by adjusting the sizes of hidden layers in the networks (see Table 14).

Each encoder learns a mapping for extracting features from the input. Autoencoder tunes the weights of each neuron in responding to a particular visual feature. A plot of the weights for the first encoder in network "7500-1500-300" is shown with Figure 34. The visual features represent various types of human body silhouettes. For the same network, the weights for the second encoder is shown with Figure 35. The visualization does not show apparent visual features as the data are further compressed down the layers.

The most time-consuming part of the training process comes from fine tuning the whole deep auto-encoder network, followed by training the first encoder. For example 


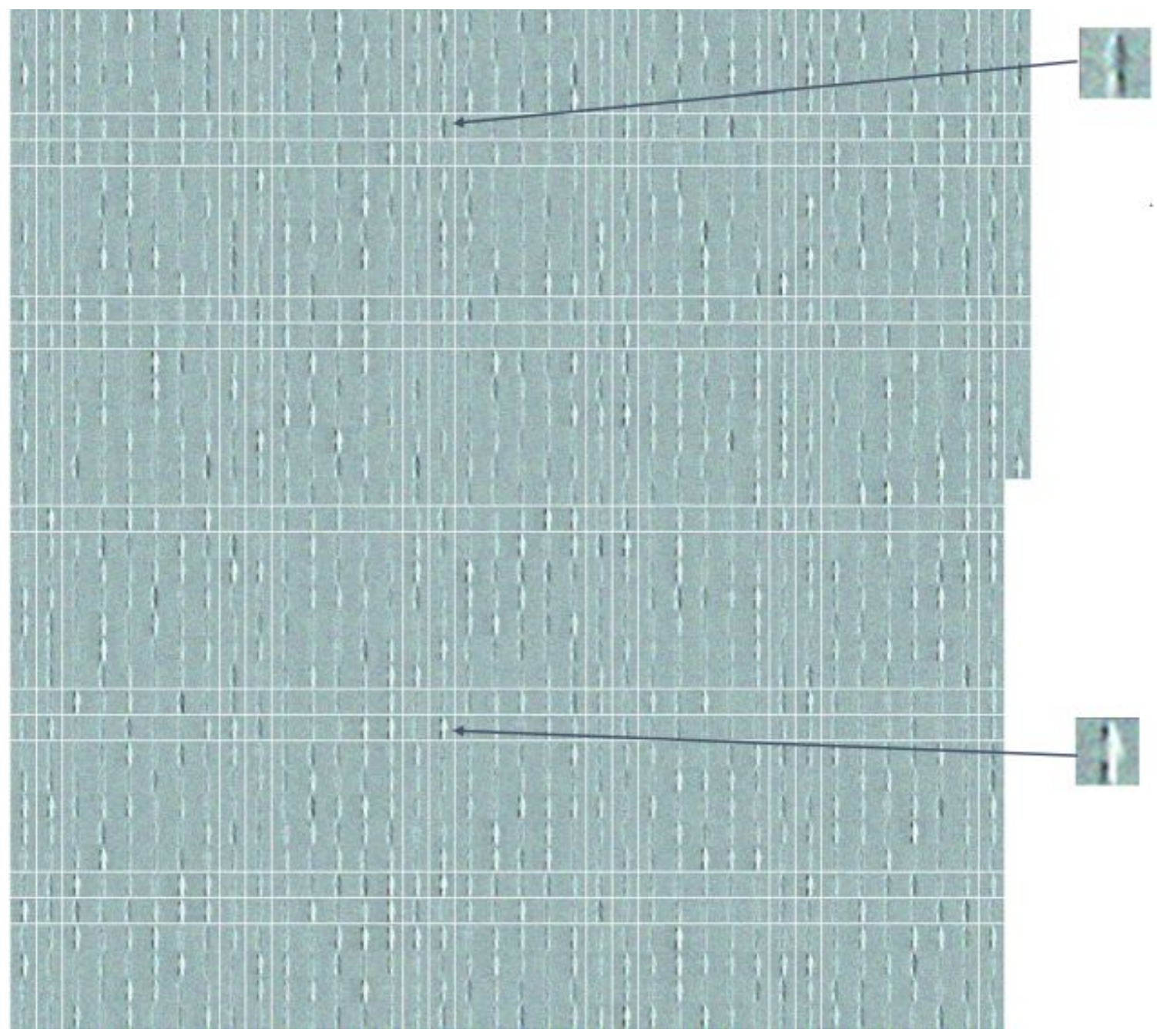

Figure 34: Visualization of features learned by the first encoder.

in training the network "7500-5000-2000", the times are one hour for the first encoder, 15 minutes for the second encoder, 6 minutes for the softmax layer, and three hours for fine tuning the whole deep network.

\subsubsection{Deep Convolutional Neural Network and SVM}

Using the pre-trained AlexNet, we select first part of the network until one of the hidden layers (any layer between conv1 and $f c 8$ ) as the feature extractor (labeled 


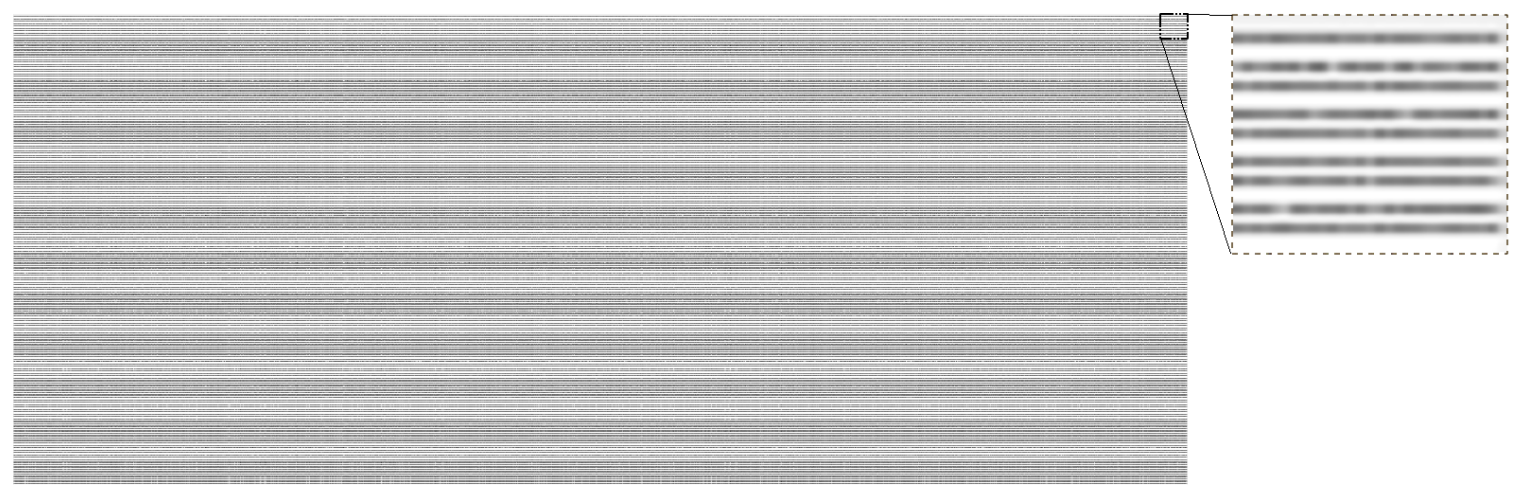

Figure 35: Visualization of features learned by the second encoder.

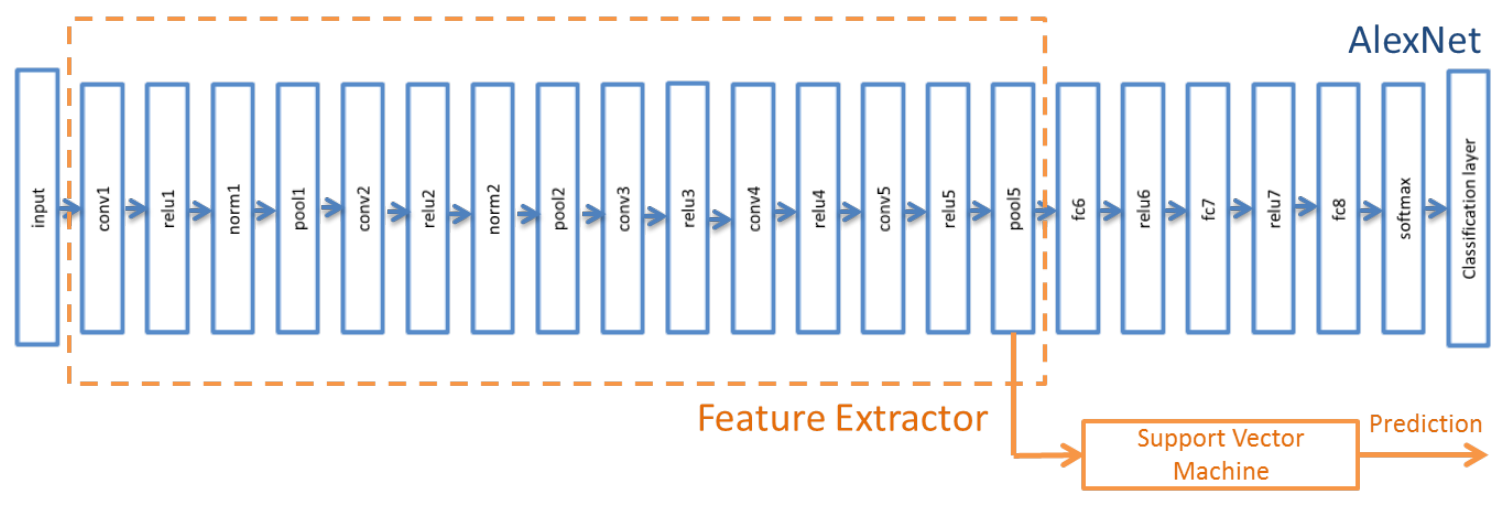

Figure 36: An example selection of first part of the AlexNet (conv1 - pool5) as feature extractor for building an SVM classifier.

within the orange dotted rectangle in Figure 36 is an example of the selection: from conv1 to pool5) for building a linear SVM classifier. The classification rates over the selection of different CNN layers are plotted in Figure 37. We draw from the graph that the best rate is achieved when the selected CNN starts from input layer and ends at layer pool5. With the dimension of feature vector at 9,216, we achieve the best classification rate at $92.14 \%$.

In general, the classification rate increases over the depth of the layers and the best performance is achieved at an intermediate level. The same trend was observed 


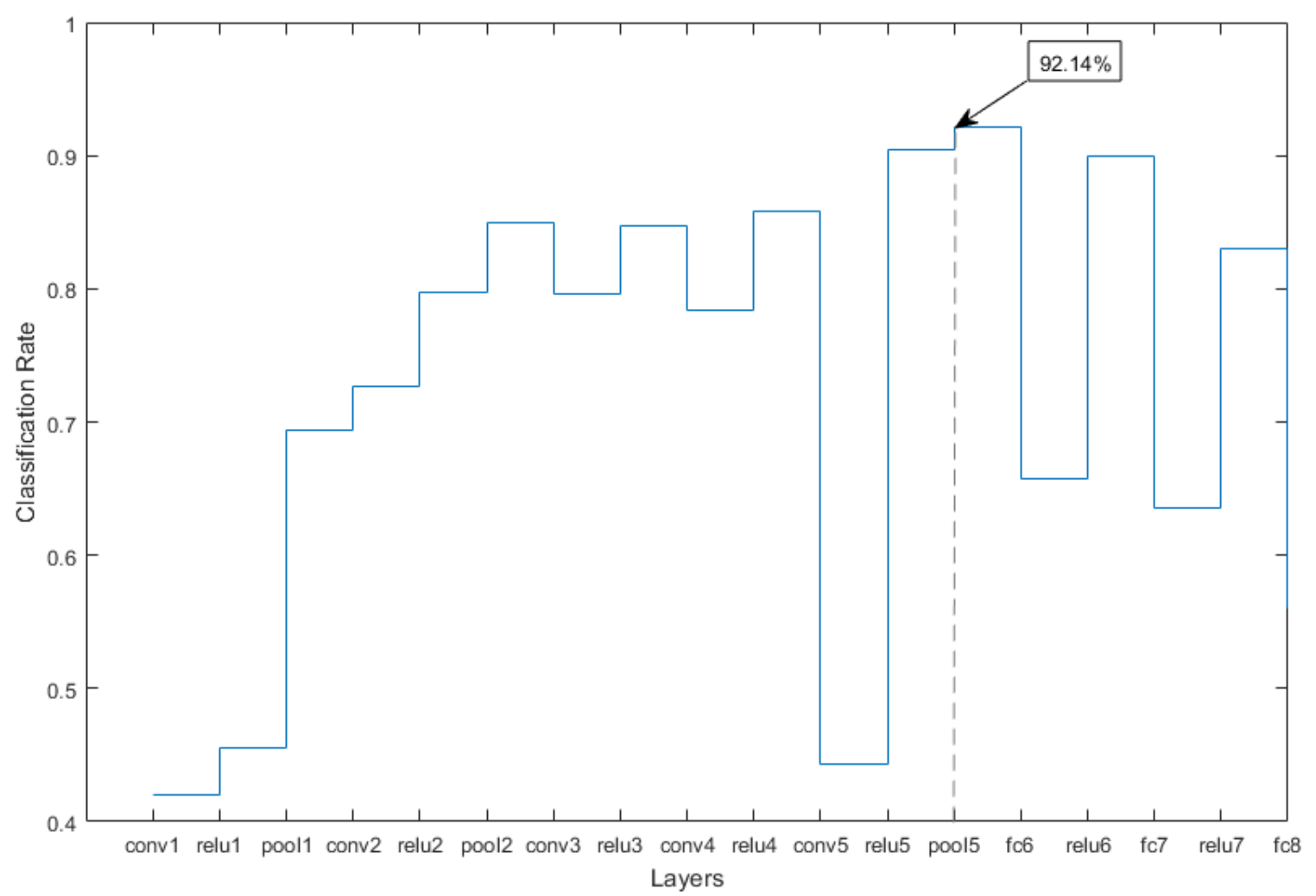

Figure 37: Classification rates over different layers in the deep CNN

in previous work on feature transferability [10,41]. Another explanation comes from Razavian et al. [41], who stated that intermediate level provides generic features while the deeper level tends to extract features specific to training images and the task trained for. Performance drops found at certain convolution layers (conv3, conv4, and conv5) are partially due to the data compression, whereas non-linear layers (relu1, relu2, relu3, relu4, and relu5) contribute to the performance boosts.

The original AlexNet took two days to train on a CPU and six hours on a GPU. With new improvements on hardware (faster GPUs and clusters) and software (more efficient ConvNet implementations), the times are reduced by a magnitude. Comparing to the time on training the AlexNet, the time on feed-forward feature extraction and classifier building is minimal (several minutes). 
Table 15: Performance comparison on image classification using different approaches.

\begin{tabular}{|l|c|c|c|c|c|}
\hline & HOG + SVM & Auto-encoders & AlexNet+SVM & AlexNet & ResNet \\
\hline Class. Rate & $50.71 \%$ & $83.14 \%$ & $92.14 \%$ & $\mathbf{9 8 . 0 0 \%}$ & $97.86 \%$ \\
\hline
\end{tabular}

\subsubsection{End-to-End Training using Deep CNNs}

In the end-to-end training approach, we compared two deep CNN models, namely AlexNet and ResNet, on their performance of human recognition. They were chosen because AlexNet was the first deep CNN model that won the ImageNet competition while ResNet is one of the recent winners.

The parameter settings for fine-tuning the AlexNet and ResNet are as follows. For AlexNet, we use the stochastic gradient descent with momentum (SGDM) optimizer. A mini batch size is set at 16 with a learning rate at $1.0 e-4$ and maximum epochs at 40. For ResNet, we use the same SGDM optimizer and set mini batch size at 32 with a learning rate at $1.0 e-3$. The maximum epochs are set at 100 .

\subsubsection{Comparison on Performance}

A confusion matrix provides too much details as the number of rows and columns will both be 100. Instead, we compute the percentage of correctly classified images (the overall identity classification rate) for each method. The comparison is listed in Table 15. The results indicate that, deep learning models have achieved higher accuracy than those using traditional features. Within the deep learning approaches, training the models end-to-end leads to better performance than using them as feature extractors only. Our best result was achieved by the AlexNet model at $98.00 \%$. 


\subsection{Discussion}

Our experiments demonstrate that the deep CNN outperforms the other two approaches in the identity classification of full-body images. Among the three methods, deep neural networks consume more time in model training; however, the rapid progress in multi-GPU and cluster-based approaches will significantly reduce the time.

The HOG feature was selected for this study. Our future work includes the Local Binary Pattern (LBP) feature. Increasing the dimension of the last encoder in the deep auto-encoders network helps improving the performance on identity classifications until certain point, after which the performance drops.

As we have limited amount of training data, we use transfer learning along with a pre-trained deep CNN. A transfer learning approach trains a base network and copies the first part of it to the target network. The remaining part of the target network is fine-tuned to its target task and the copied part remains frozen during fine-tuning. This works especially well in avoiding over-fitting in cases when the target dataset is small and the number of parameters is large [10]. The classification rate using this approach proves the great performance boost from learning transfer.

We provide the visualizations of features computed using HOG and auto-encoders. The first encoder layer represents richer visual information in the training images than the HOG. Because each encoder captures strong, high-order correlations between the activities of units in the layer below, the visualization of the first encoder demonstrates body-like silhouettes from the input layer. Our observations on the second encoder agrees with [34], which states that the following encoders in the network progressively reveal low-dimensional, nonlinear structure. These progressive representations of features show an opposite trend from those observed in the deep convolutional networks, where the first several hidden layers represent primitive image features and the deeper layers represent more complex features composed of the primitive ones [116]. 
We study different feature extractors on their robustness to viewpoint changes in visual classification applications. We compute features and train classifiers on certain viewpoints and test their capabilities on new viewpoints. The features computed using a deep CNN achieve the highest classification rate on novel views. The current experiments make an assumption that the testing cases belong to one of the training classes. To build a practical access control system, the testing cases may not belong to any of the training classes. In this situation, the classifiers need to compute a distance or score of the testing cases when using the classifiers. Proper thresholds need to be set in order to avoid over-prediction or under-prediction [117].

To build a practical access control system, one needs to consider other factors, including human in cluttered background, human in different poses, human in various appearances, and the quality of images taken. To address these issues, a more complete training dataset covering these variances is necessary for building the classifiers. In this work, we focus on computing the best viewpoint-independent features and the same approach can be extended to other datasets with variations aforementioned.

\subsection{Conclusion}

In this chapter, we validate the proposed deep learning framework on 3D human data. In particular, we empirically study the viewpoint independence of 2D image features. We implement different feature extractors for the identity classification of multiple-view full-body images. Using traditional feature extraction method as a baseline, we demonstrate that automatic feature extractions achieve better performance. We also demonstrate that the combination of a pre-trained deep CNN and learning transfer outperforms the auto-encoder-based deep neural networks. Further experiments indicate that end-to-end training of the deep CNN models leads to even better performance than using them solely as feature extractors. 


\section{Chapter 6}

\section{Representation Learning on 3D Data for Locating Landmarks}

There are more applications in 3D than human recognition; therefore in this chapter, we continue the introduction of our proposed framework in $3 \mathrm{D}$, through extending the capabilities of deep neural networks. The framework is applied for predicting anatomical landmark locations on 3D human surface data. This is an important step for 3D human data analysis and a non-typical task for existing deep CNN models. In the proposed deep learning framework, components used for the validation are highlighted in Figure 38.

Anatomical landmarks play key roles in many 3D applications that involve 3D human body scans, including consistent parameterization, body measurement extraction, segmentation, and mesh deformation. Manually locating landmarks is tedious and time-consuming for large-scale 3D anthropometric surveys. To avoid this laborintensive work, we propose a data-driven approach, which learns from a dataset of 3D scans with known landmark locations and predicts their locations on new scans. More specifically, we adopt a coarse-to-fine approach by applying a deep regression neural network to computing the locations of selected landmarks and then employing 


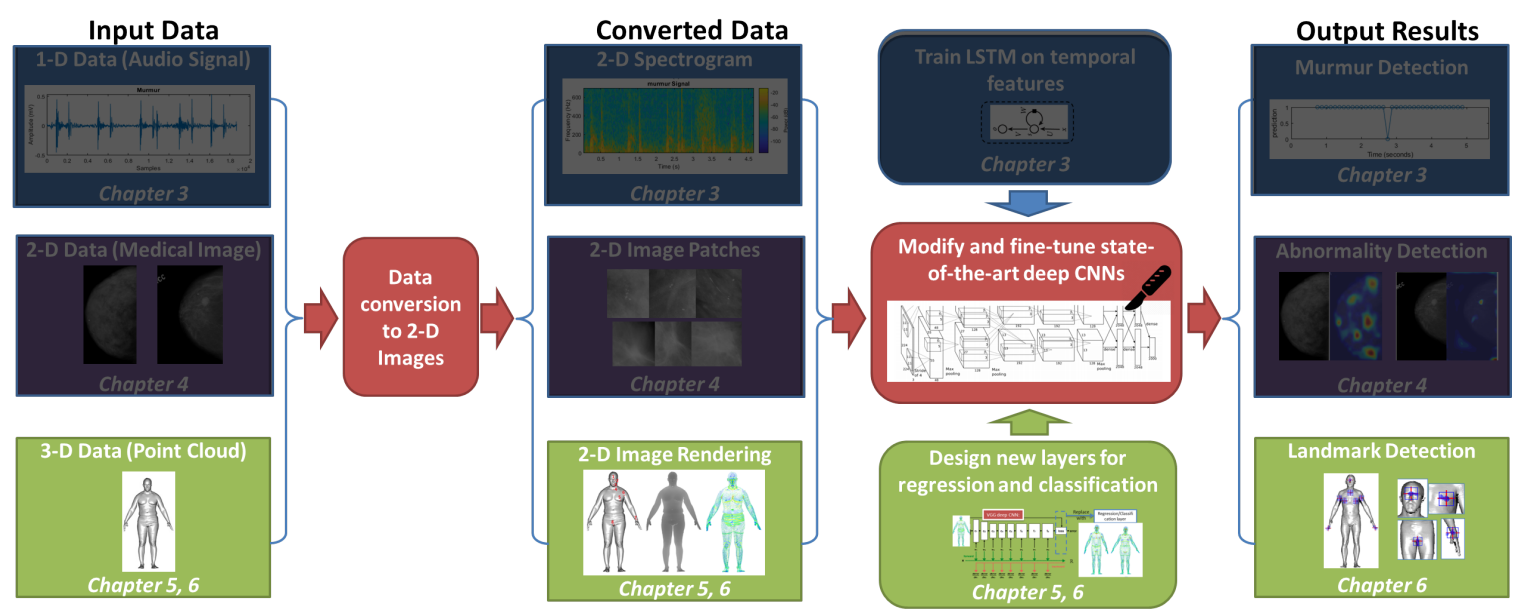

Figure 38: Applying the deep representation learning framework on 3D human surface data for locating landmarks (from left to right: raw data, converted 2D data representations, deep model training and output).

for each landmark an individual deep classification neural network to improve its accuracy. Moreover, we compute from a frontal view and compare three types of image renderings for training the deep regression network, i.e., appearance images, range depth images, and curvature map images. Among them, depth images result in the best empirical accuracy on landmark predictions and therefore are selected to train the deep classification networks. Our proposed approach achieves close-to-human accuracy on locating landmarks and can be extended to automate the landmark locating process for large datasets of 3D human body scans.

\subsection{Introduction}

Accurate 3D anatomical landmarks are of great importance for shape-essential applications, including consistent parameterization, body measurement extraction, segmentation, and mesh re-targeting. In large-scale anthropometry surveys, manually 
locating these landmarks is a time-consuming and tedious process.

In this chapter, we propose a method using deep Convolutional Neural Networks (CNN) for automatically locating 3D anatomical landmarks for a large database of full-body scans. A 3D scan consists of a collection of unordered 3D points. Different scans have different number of points. Without pre-processing, this type of data is not suitable for the current deep CNNs.

Although there have been recent work on using deep CNNs with 3D clean meshes [118] [119], the localized geodesic convolutional neural network will fail to work on

3D raw scans because of data imperfections (noise, holes, surface flipping, etc.). We tackle this problem by rendering $3 \mathrm{D}$ scan data into different types of $2 \mathrm{D}$ images for use in the deep regression and classification neural networks. More specifically, we employ a deep regression $\mathrm{CNN}$ to predict rough landmark locations using full-body images, followed by deep classification CNNs to improve their accuracy using local image patches.

State of the art [120] has a mean localizing error between $1.2 \mathrm{~cm}$ and $2.6 \mathrm{~cm}$ on different 3D anatomical landmarks. We aim to outperform the state of the art.

\subsubsection{Accurate 3D Landmarking}

It is necessary to note that the problem of locating anatomical landmarks has more stringent accuracy requirements than the current object localization tasks in ImageNet Large Scale Visual Recognition Competition (ILSVRC), where the size and location of bounding boxes allow some tolerances [2].

Many downstream processing on 3D scan data relies on the accurate locations of the landmarks. 3D consistent parameterization relies largely on the accurate locations of landmarks, which guide template mesh deformation and ensure the correspondence of key anatomical features after parameterization [121] [122]. The 3D consistent parameterization can be used to compute deformable shape space for data-driven 
applications [123] [124] [125].

Anatomical landmarks are used in extracting body dimensions from 3D scans where they provide exact location and delineation for the measurements. This makes it possible to extract arbitrary body measurements from the scans, providing richer information than traditional tape measures. Again, the accuracy of measurements relies on the accurate locations of 3D landmarks.

3D landmarking also plays essential roles in computer vision and animation. In 3D imaging, surface matching relies on efficiently computing accurate landmarks for mesh alignment. Computer animation techniques such as skeleton-driven approaches depend on 3D landmarks for creating realistic human animation effects. Mesh retargeting is another example that relies heavily on landmarks.

\subsubsection{Deep Convolutional Neural Networks}

Recent years witnessed great performance advancement on image benchmark tasks using deep convolutional neural networks. Comparing to traditional hand-crafted image features, deep CNNs automatically extract the best features from a large dataset for tasks they are trained for. For example, the best-performing models in recent ImageNet challenges have used very deep CNNs [2] [9] [126]. However, due to the complexity of 3D scan data, there have been few published work on using deep CNNs [127] [128].

Our experiments and tests are conducted on the CAESAR database [113]. More specifically, we make orthogonal projections of 3D human body scans onto front-view image planes using three different representations: model appearance rendering with lighting, range image with depth projection, and image rendering showing curvature mapping. We compare these three image sources and search for the best one for predicting landmarks.

We conduct landmark predictions in two steps. First, we use a deep regression 
CNN to compute the locations of all landmarks. Second, for each landmark, we train a deep classification CNN using image patches centered at the landmark and at nearby pixels, termed phantom landmarks, to build distinguishing classes for the current landmark.

Our contributions are three folds. First, we convert 3D raw scan data to 2D image representations that are best suitable for the use of deep CNNs. Second, we compare three different sources of images by training deep regression networks and discover the best one for landmark prediction. Third, we improve the accuracy of landmark locations by training deep classifiers.

\subsection{Related Work}

Object detection is a challenging task in computer vision. Traditional approaches for object detection compute hand-crafted features and then train a shallow classifier such as SVM or logistic regression. In using deep-learning approaches, image classifiers are trained to automatically learn features and work on sliding windows across an image [42] [97] [99] [129]. One good example is RCNN [129] [130], which makes region proposals using selective search instead of doing exhaustive search.

Recent work on object detection argues that simultaneously learning on multiple tasks can improve the performance on each individual task [126] [103] [131]. The work from Li etc. [131] simultaneously learns a human pose regressor and sliding-window body-part and joint-point detectors in a deep network architecture. They frame pose estimation as a regression task, while also defining two accessory tasks to guide the network to learn useful features for pose estimation. In particular, these accessory tasks are sliding window detectors for various body parts and joints. It is shown that joint training improves the learning speed and increases the accuracy of pose regression. 
Object detection also depends on image sources. Predicting human pose from depth images proved to be more robust than from 2D images [132]. With 3D human body models, we can set up projection matrices for comparing different image sources on landmark prediction.

Current 3D landmarking approaches rely on traditional hand-crafted feature computations [120,133]. Giachetti etc. [120] introduced six different methods on automatically locating 3D landmarks on human body scans. These methods computed hand-crafted feature representations for learning and predicting landmark locations. More specifically, features include histogram-based SPIN image, spectral geometry based Laplace-Beltrami operator (LBO) and augmented point feature histograms (APFH). Wuhrer etc. [134] introduce 3D posture-invariant shape features with SPIN images for creating the shape descriptor.

There are more published work on identifying landmarks on 2D images than on 3D meshes; however, 2D human images have to deal with large variations on posture, lighting condition and resolutions. Vezzetti and Marcolin [135] claim that the most straightforward way of locating landmarks is viewing it as a regression problem. A significantly deep network was used for predicting landmarks. Fan and Zhou [136] use cascaded deep CNN for facial landmark localization and formulate it as learning a function that maps image pixel arrays to point coordinates.

\subsection{Deep Learning for Landmark Prediction}

In this work, we focus on computing the accurate locations of landmarks by combining a deep regression $\mathrm{CNN}$ with deep classification CNNs. We employ a coarse-to-fine approach by predicting landmark locations using a regression network for holistic feature computation and then improving their accuracy using multiple deep classification networks. Comparing to $2 \mathrm{D}$ images, the one extra dimension in 3D data 
enables computing unique 3D features for training deep CNNs.

Transfer learning [10] has been a popular approach on applying pre-trained deep neural networks to a small dataset in order to avoid over-fitting. Starting from a deep CNN pre-trained on a large set of data, the model can be customized to work with a different and small set of training data for fine fitting. This helps to address the challenge on training deep neural networks with limited number of training data. In this work, we adapt a pre-trained generic deep CNN to the regression and classification tasks.

\subsubsection{Data Preparation}

The CAESAR dataset (CAESAR - Civilian American and European Surface Anthropometric Resources) is a collection of 3D full-body laser scans with manual landmarks and measurements. For each body scan, a set of 73 landmarks were manually palpitated and labeled by anthropometrists during scanning. The 3D locations of these landmarks were then identified and recorded using a 3D software. For this work, we select eight landmarks which are visible from front-view projections (see the left image in Figure 39). Without loss of generality, these landmarks are located on the left side of human body.

For each body scan, we use an orthogonal projection matrix to project the 3D model onto a 2D image plane. Firstly, we light the 3D model with an upfront light source and render the image to compute the gray scale appearance image. Secondly, we compute the depth image and map it onto the 2D image plane. Thirdly, we compute Gaussian curvature maps on the 3D model, color-code each vertex with curvature values, and then project the curvature map onto the 2D image plane. Figure 39 demonstrates image examples computed using the three methods.

The selected 3D landmarks are mapped onto the 2D image plane using the same 

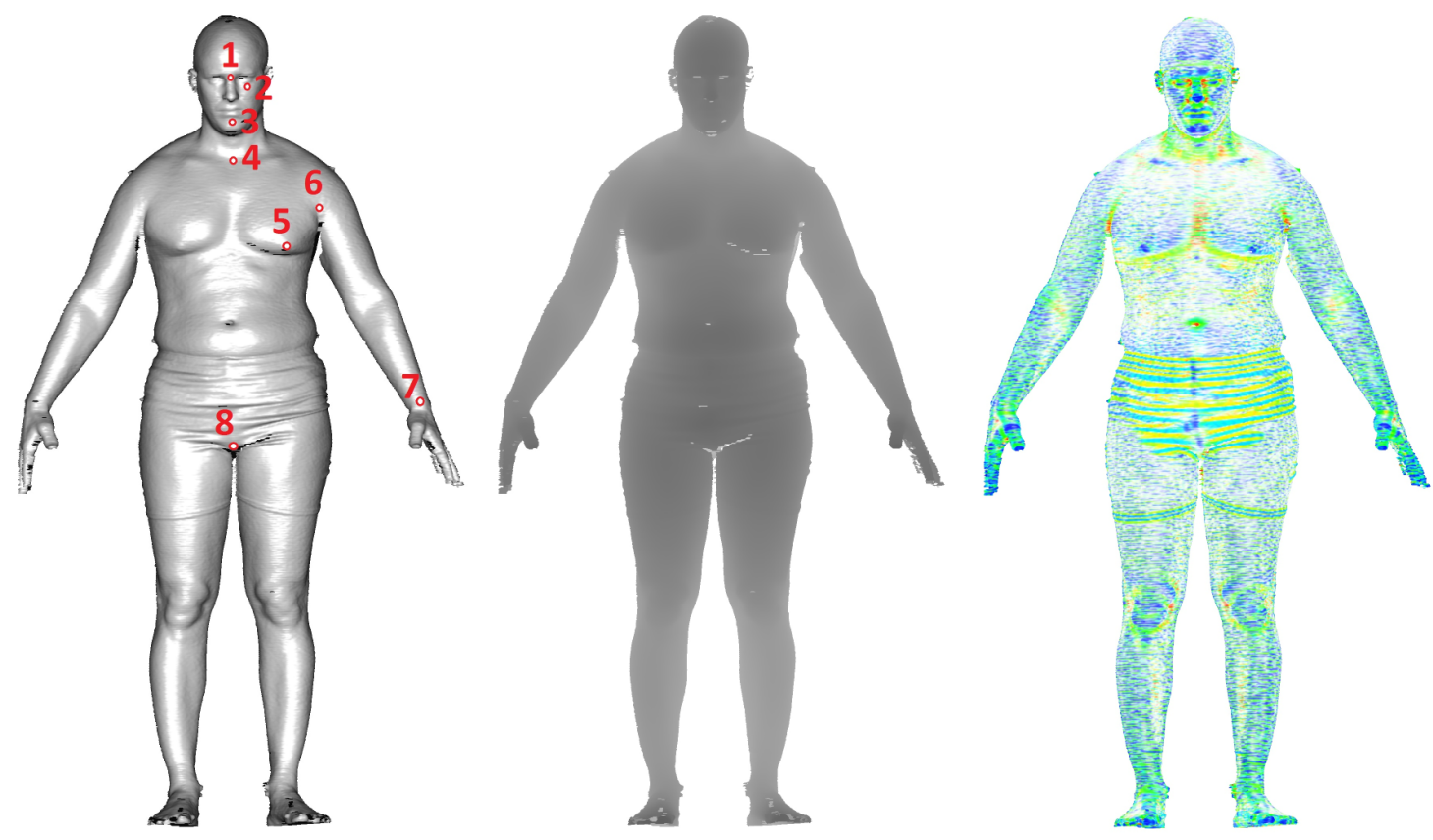

Figure 39: From left to right: rendered appearance image (eight selected anatomical landmarks are labeled in red), range depth image, and curvature map image. The landmarks are: 1-Sellion 2-Infraorbitale 3-Supramenton 4-Suprasternale 5-Thelion 6-Anterior Axilla 7-Radial Styloid and 8-Groin.

projection matrix as used by the image projections. In training the deep neural networks below, the inputs are the images and the outputs are a vector of concatenated $2 \mathrm{D}$ locations of the landmarks.

\subsubsection{Deep CNN for Regression}

We first customize VGG network [137] to a deep regression network for predicting the locations of all eight selected landmarks. The VGG network secured the first and second places in the localization and classification tasks in ImageNet ILSVRC-2014 challenge. It is comprised of five convolutional layers followed by three fully-connected layers. To modify it for doing regression, the last softmax layer is removed and the 
last fully-connected layer is changed to match the size of the output, which is twice the number of selected landmarks.

A customized layer is appended to the modified network for computing losses. In this work, least square error $\left(L_{2}\right)$ is used for forward loss computing and backward loss propagation.

\subsubsection{Deep CNN for Classification}

The deep regression network computes the locations of the eight selected landmarks from a full-body image. For each individual landmark, we train a deep classification network to further improve the accuracy of location prediction. For each landmark, we select nearby pixels called phantom landmarks for extracting training image patches to build a classifier.

To modify the VGG network for this purpose, the output softmax layer is first removed and the last convolution layer is changed to reflect the size of the output, which is five, the number of classes for the new classifier. A new softmax loss layer is then appended to the customized network for the classification of image patches.

All the parameters and weights are inherited from the published VGG network, except learning rate is adjusted to ensure the convergence of learning processes.

The deep regression CNN predicts locations of all the selected landmarks. Setting a reasonably large searching area around each predicted landmark and using sliding window approach with the deep classification CNN results in the best predictions of landmark locations.

\subsection{Experimental Results}

We customize and train the deep CNNs using MatConvNet, a MATLAB toolbox for Convolutional Neural Networks [138]. The toolbox provides basic building blocks 
of a deep CNN: convolution, pooling, and non-linear activations. MatConvNet also has a repository of pre-trained models that can be readily loaded into MATLAB for fine-tuning with new datasets. It supports the use of multiple GPUs.

\subsubsection{Data Preparation}

After data cleaning, a set of 2,378 body scans and their landmarks are selected from the CAESAR database. Following a common data splitting practice in machine learning, we randomly select $20 \%$ of the whole dataset for testing and the rest for learning, which is then further split into two sets - $80 \%$ for training and $20 \%$ for validating. This leads to 1522,380 , and 476 scans in the datasets for training, validating, and testing purposes. Validation datasets are used to avoid over-fitting during learning processes.

After rendering and projection, the size of rendered images are all [2240, 2240,3]. Due to the requirement on input image sizes from the VGG network, all the images are scaled to $[224,224,3]$ and then normalized. Moreover, landmark coordinates are projected to $2 \mathrm{D}$ and then scaled to match the downsized images and then normalized.

Examples of rendered appearance images, range depth images and curvature map images are shown in Figures 40, 41 and 42, respectively.

\subsubsection{Deep Regression CNN}

Feeding the pre-processed images and landmark data into the customized deep regression CNN starts the training of the deep regression CNN. The cost function curves on training and validation data sets turn flat after training for about 40 hours with 16, 000 epochs. The learning rate is set at $0.5 e-4$. 


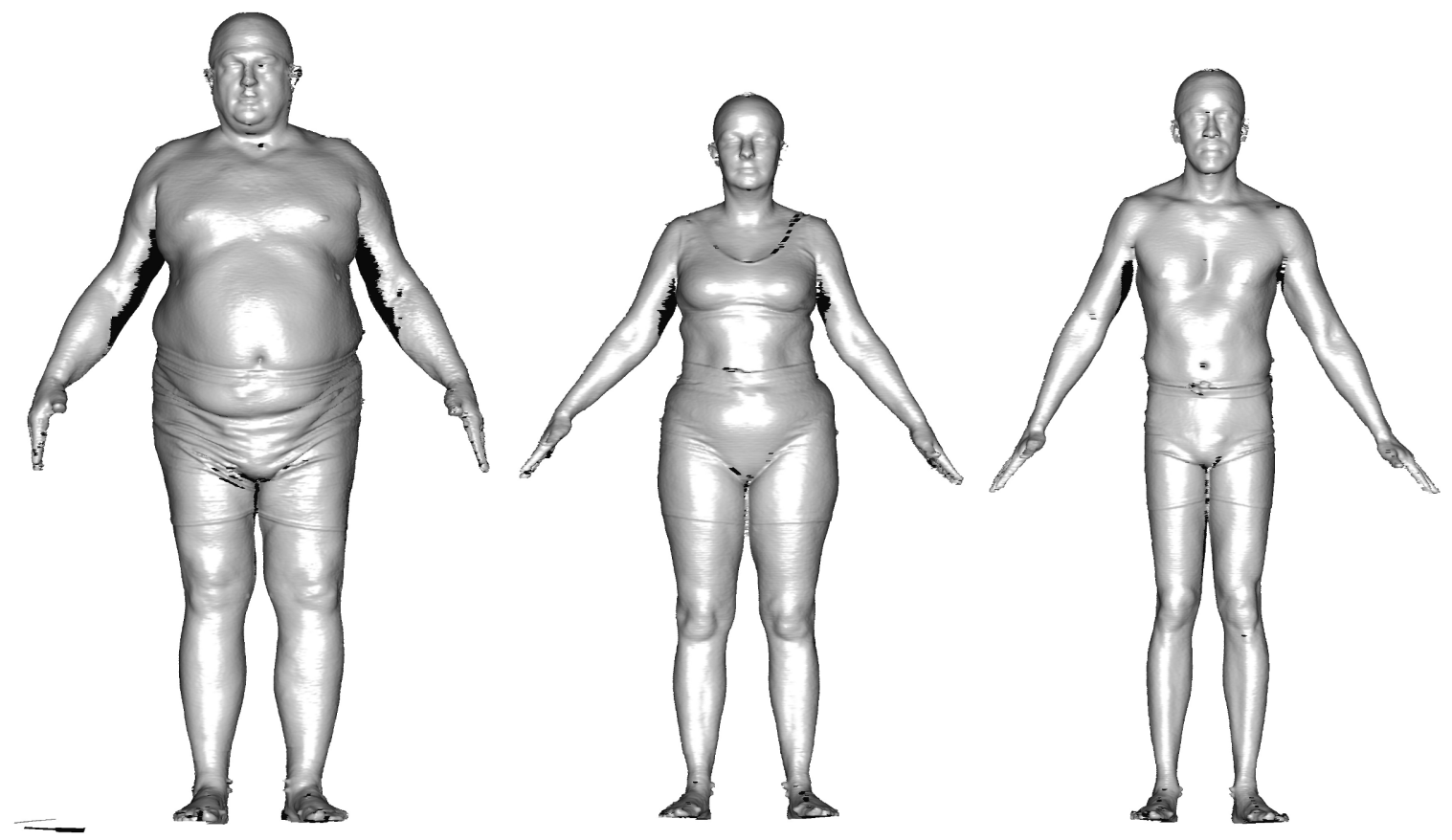

Figure 40: Examples of rendered appearance images.

\subsubsection{Deep Classification CNN}

The deep regression CNN computes the locations of the selected eight landmarks from full-body images. To improve the result, we train a deep classification network for each landmark. More specifically for each landmark, we use the landmark and its four-corner nearby phantom landmarks, which are 20 pixels away on x-axis and y-axis. Figure 43 illustrates an example of the selection of such phantom landmarks for the sellion landmark. Square image patches of size $[80,80]$ centered at each of the five landmarks are extracted and saved, leading to one positive and four negative image patches for each landmark on every training subject in order to learn a classification CNN.

The same image patch extraction approach is applied to other selected landmarks for training separate deep classification CNNs. Training the deep classification CNN on depth images takes about 18 hours for completing 1,800 epochs (learning rate set 


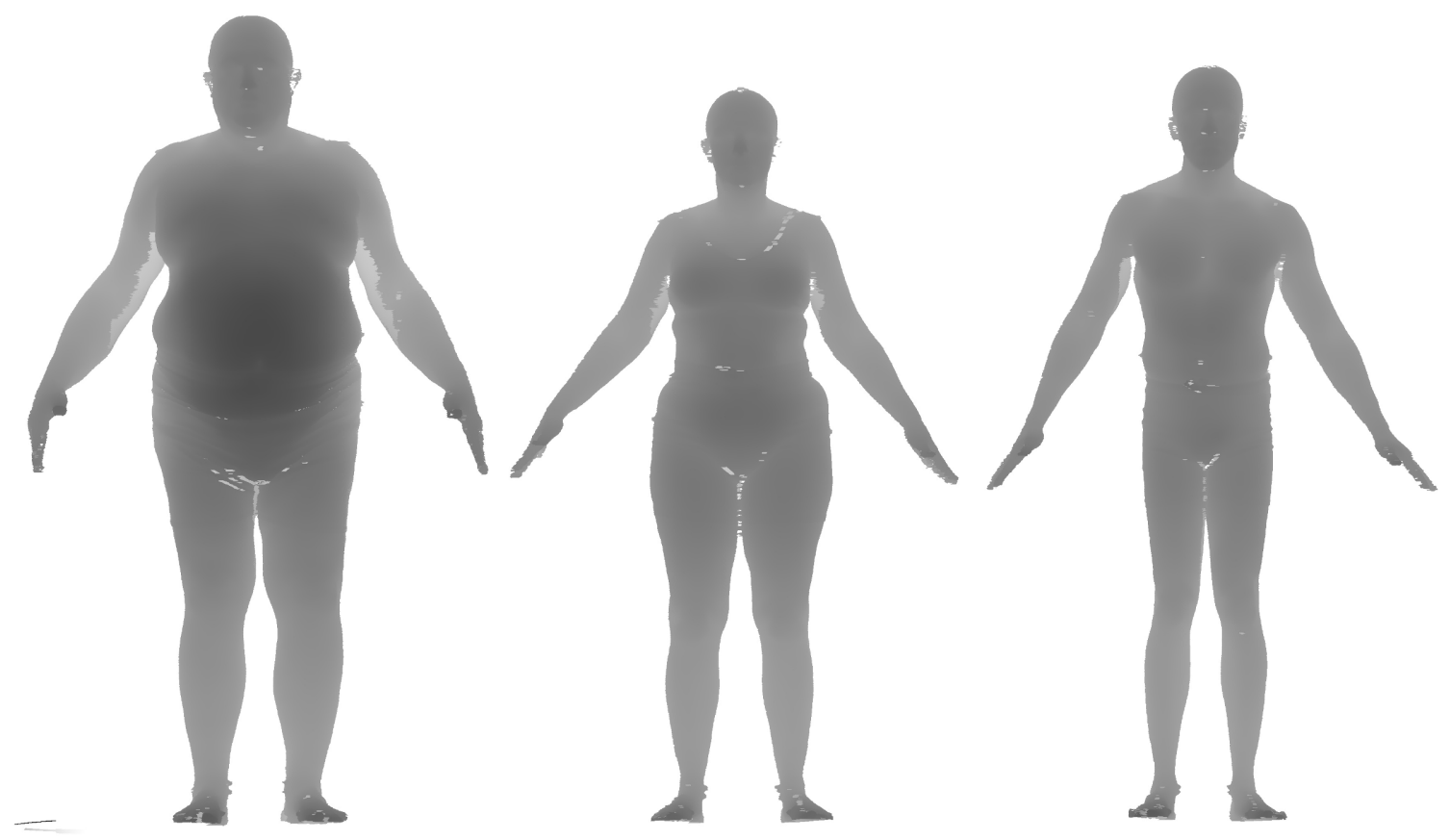

Figure 41: Examples of range depth images.

at $2.0 e-5)$. Training the deep classification $\mathrm{CNN}$ on curvature images takes about 24 hours for completing 400 epochs (learning rate set at 1.0e-4).

\subsubsection{Prediction Results}

At the end of training the deep regression network, we compare the prediction errors on each landmark for the three image sources (see Figure 44). Using curvature map images as the source for training and predicting landmark locations results in the lowest errors on almost all the landmarks. A few sample regression results are illustrated with Figure 45. As one may find, despite different heights, weights, shape and even arm postures, the deep regression network performs well in predicting the eight landmarks' locations.

Regression results are then used to define a local search window for each landmark. After learning a deep classification CNN for each landmark, small image patches 


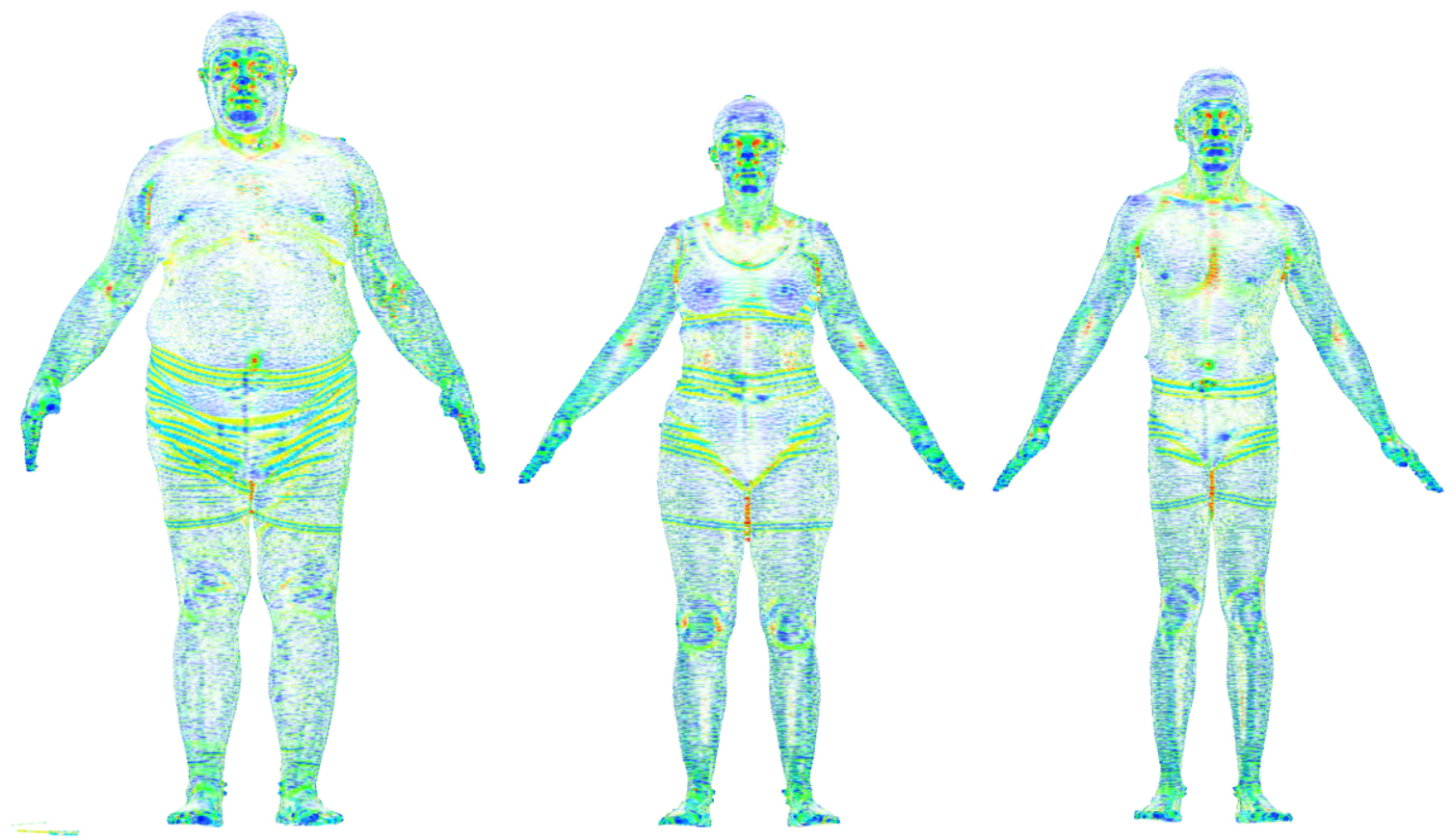

Figure 42: Examples of curvature mapped images.

(same size as those used in training) are extracted for classification. In most cases, multiple image patches (candidates) are classified as the landmark patch other than the phantom patches; however, only the center of an image patch with the highest classification probability is considered as the final location of the predicted landmark.

Using the sellion landmark as an example, a sweeping-window approach (step size of 5 pixels) is used to classify each extracted image patch surrounding the landmark (searching area size $[100,100])$. Figure 46 shows a few examples of the classification results. Other landmark prediction results are demonstrated with examples shown in Figures 47 to 53. The classification is performed on depth images and the predicted landmark is marked on appearance images for better visualization. The results can be further improved with a reduced step size of the sliding window.

These figures provide visual evidence that the deep classification CNNs perform very well in finding the location of each landmark. In the visualization, each image 


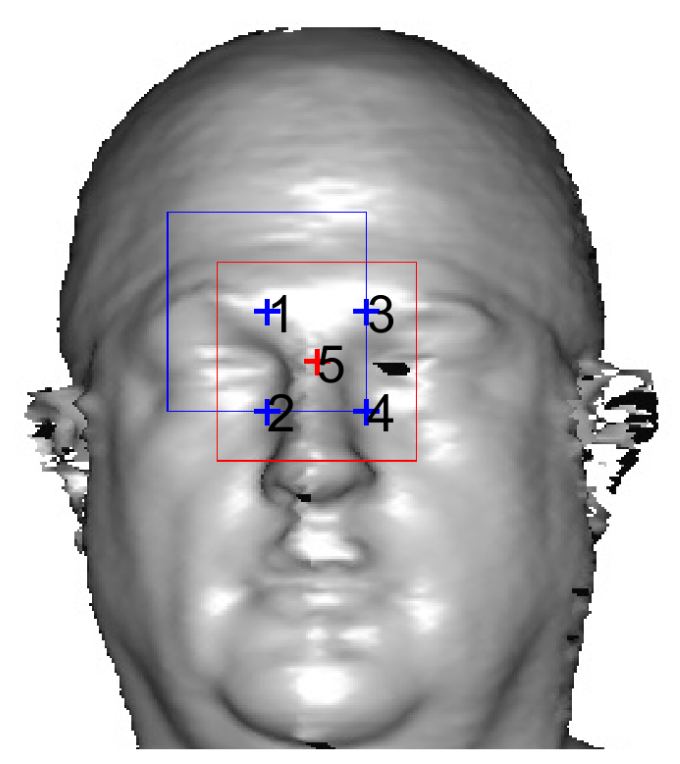

Figure 43: Phantom landmarks selection (red cross (5): current sellion landmark; red square: image patch extraction area around current sellion landmark; blue crosses (1-4): phantom landmarks on four corners; blue square: image patch extraction area around the first phantom landmark).

icon shows the searching area in blue squares. Blue crosses are the candidate landmark locations, where the extracted patch is classified as the landmark. Among all the candidate locations, the one returning the highest probability during classification is labeled with red cross to indicate the final location of the predicted landmark.

A quantitative comparison with the deep regression CNN is conducted on the prediction results from the deep classification CNNs (Table 16). Comparing to the results from regression on curvature, better prediction results on landmarks using the classification CNNs (depth or curvature) are highlighted in bold.

Based on a sample 3D linear measurement (parallel to the image plane) and its length in the $2 \mathrm{D}$ image plane, one pixel in the $2 \mathrm{D}$ image corresponds to $0.9 \mathrm{~mm}$ in $3 \mathrm{D}$ space. As one may find, the deep CNNs on depth images achieve better performance 


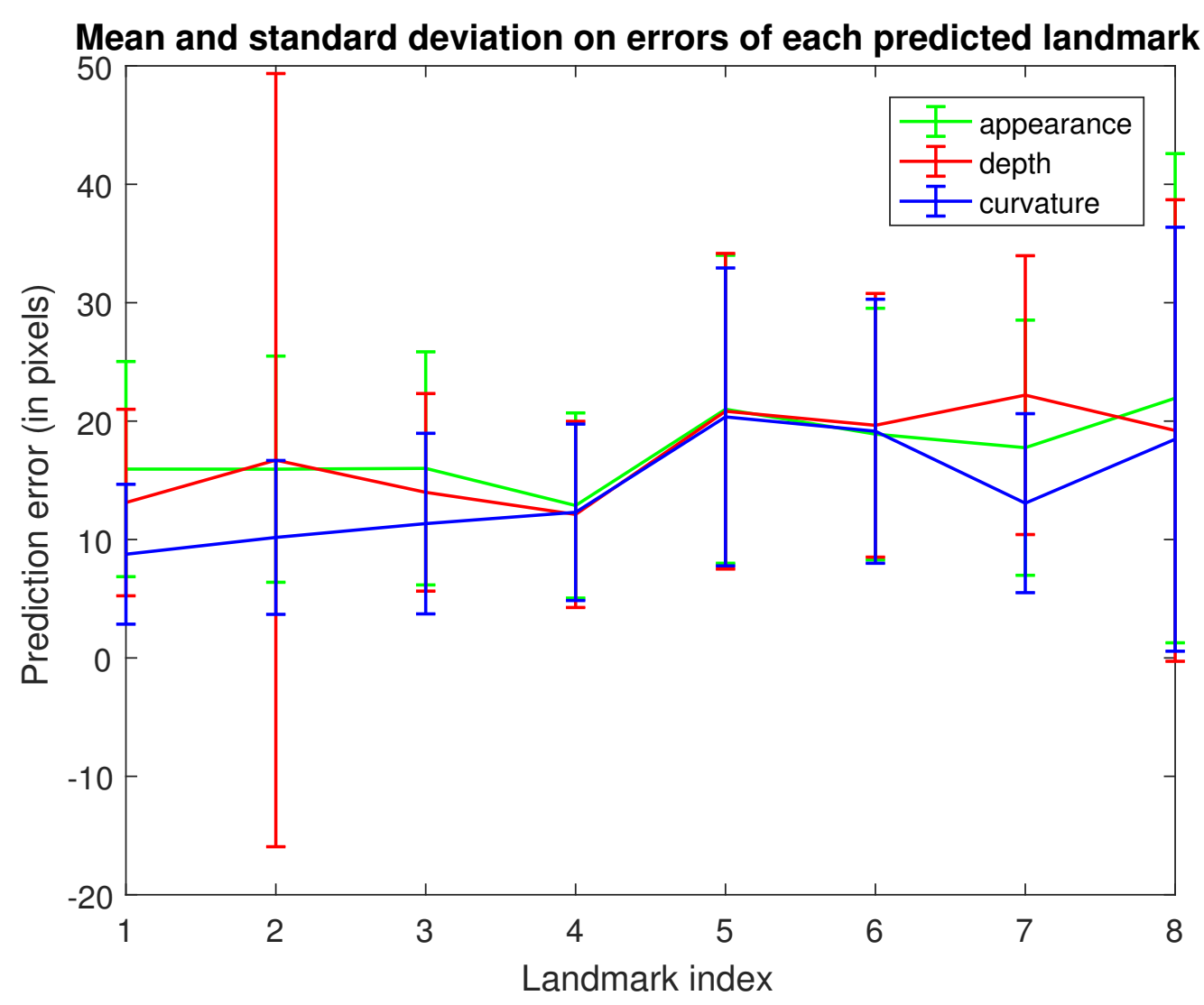

Figure 44: Comparison on mean and standard deviation of prediction errors on each landmark.

than state of the art [120] on most landmarks except on 'Land. 6 - Anterior Axilla' and 'Land. 8 - Groin'. In comparison, the classification CNNs on curvature maps achieve better accuracy on 'Land. 5 - Thelion' and slightly better accuracy on 'Land. 8 - Groin'.

The challenges on locating 'Land. 6 - Anterior Axilla' and 'Land. 8 - Groin' result from the raw data imperfection (mesh holes because of occlusions during scanning). In comparison, state of the art [120] uses cleaned mesh (hole filled and noise removed) for landmarking tests.

All the experiments are implemented in Matlab with GPU support (NVIDIA 


\begin{tabular}{|c|c|c|c|c|c|c|c|c|}
\hline Networks & Land. 1 & Land. 2 & Land. 3 & Land. 4 & Land. 5 & Land. 6 & Land. 7 & Land. 8 \\
\hline $\begin{array}{l}\text { Regress. on } \\
\text { Curvature }\end{array}$ & {$[8.7,5.9]$} & {$[10.1,6.4]$} & {$[11.3,7.6]$} & {$[12.3,7.4]$} & {$[20.3,12.5]$} & {$[19.1,11.1]$} & {$[13.0,7.5]$} & {$[18.4,17.9]$} \\
\hline $\begin{array}{l}\text { Class. on } \\
\text { Depth }\end{array}$ & {$[5.2,3.3]$} & {$[7.7,4.0]$} & {$[7.9,5.7]$} & {$[11.2,7.9]$} & {$[10.3,9.7]$} & {$[23.5,15.4]$} & {$[9.0,5.1]$} & {$[16.1,12.4]$} \\
\hline $\begin{array}{l}\text { Class. on } \\
\text { Curvature }\end{array}$ & {$[18.0,16.5]$} & {$[21.9,22.3]$} & {$[8.0,6.2]$} & {$[29.9,20.1]$} & {$[6.8,7.6]$} & {$[19.9,16.2]$} & {$[11.3,10.4]$} & {$[15.7,12.4]$} \\
\hline
\end{tabular}

Table 16: Results comparison on selected landmarks between the regression network and the classification networks. The numbers in each cell represent the mean and standard deviation of the prediction errors (in pixels).

GeForce GTX Titan X). Detecting a landmark using the sweeping-window approach takes about 15 seconds, which can be improved after parallelizing the windowsweeping approach.

\subsection{Discussion}

For training a deep regression $\mathrm{CNN}$, the size of the input images is restricted by the pre-trained deep CNN. Therefore, the accuracy of the predicted landmark locations is limited. Building and training a new deep CNN allowing larger-size images may improve the accuracy of results.

Among the three image sources, appearance images are easily affected by lighting directions and intensities. When intensity is high, the reflection on mesh surfaces washes out 3D image features after rendering to 2D images.

Curvature mapping is also affected by certain parameter settings. Different scales 
used for computing surface curvatures result in different curvature values. Moreover, there are variants of computing the curvature: principal curvatures, mean curvatures, and Gaussian curvatures. In comparison, depth images are least affected by experimental settings.

The deep classification CNNs are trained using image patches extracted around each landmark and nearby phantom landmarks. The size of the extracted image patches and the number and location of phantom landmarks can be adjusted accordingly for each individual landmark. As an example, landmark 4 may need larger image patch sizes for training as it is located on a flat surface area.

As one of the manual features, curvature maps achieve the best accuracy from full-body images among the three image sources; however, when working with local patches, depth images demonstrate empirical superiority over curvature maps. This is partly because that computing manual features leads to the loss of information. Curvature maps do extremely well on locating 'Land. 5 - Thelion' as the feature computation highlights the anatomical landmark to be identified.

We experiment on predicting the locations of landmarks from front-view images on the left side of human bodies. The same approach can be extended to work on the other side of the human body and from other views (e.g., landmarks visible from the rear). The 3D locations of these landmarks can then be computed after projecting back to the $3 \mathrm{D}$ space.

The experimental results have demonstrated its robustness in dealing with imperfect scan data with holes and noise because all the training and testing have been conducted on raw 3D laser scans with minimum pre-processing. For the standing posture, the frontal and rear views for image capturing suffice for computing image features in order to accurately identify the landmarks as has been proposed. In a more complicated posture such as a seated one, a few more postures are expected for landmarks not visible from existing viewpoints. 


\subsection{Conclusion}

In this chapter, we continue the validation of the proposed deep learning framework on an important task of landmark prediction in 3D computer vision. We successfully complete the customization of a generic classification CNN to computing holistic features for landmark regression. We also customize the same network to create new classification CNNs for conducting accurate landmark predictions. The proposed framework proves its effectiveness in achieving higher accuracy than state of the art with exceptions on landmarks where surface data are missing in 3D.

Different image sources have their superiority and weaknesses in identifying landmarks. In this chapter, we conclude depth images perform the best among the three image sources. Our future work includes searching for better image sources for training the deep CNNs. We will extend the approach to identifying landmarks from different viewpoints and on human body scans in different postures. 

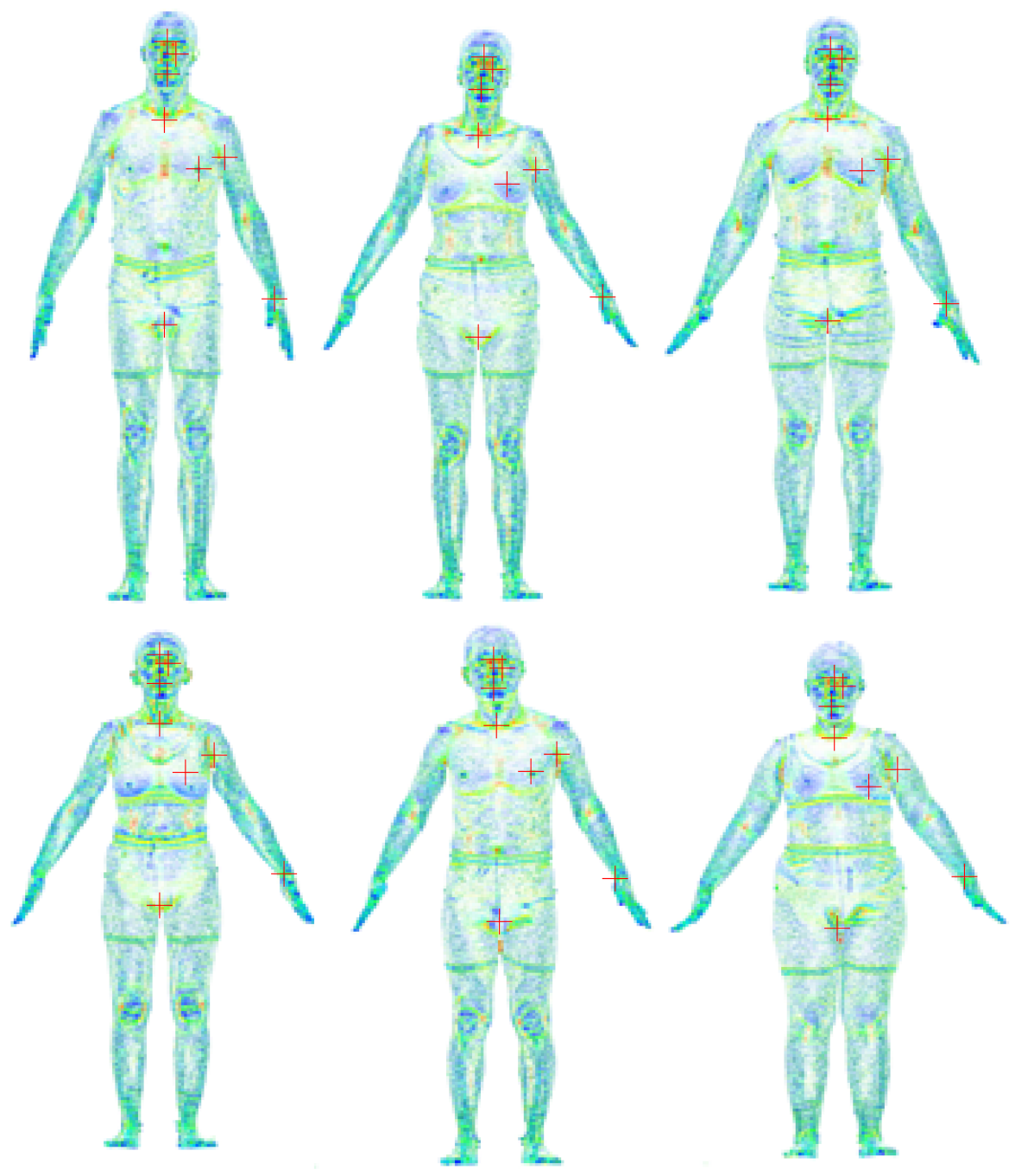

Figure 45: Regression result on sample test images (red cross: locations of predicted landmarks from the deep regression CNN. 

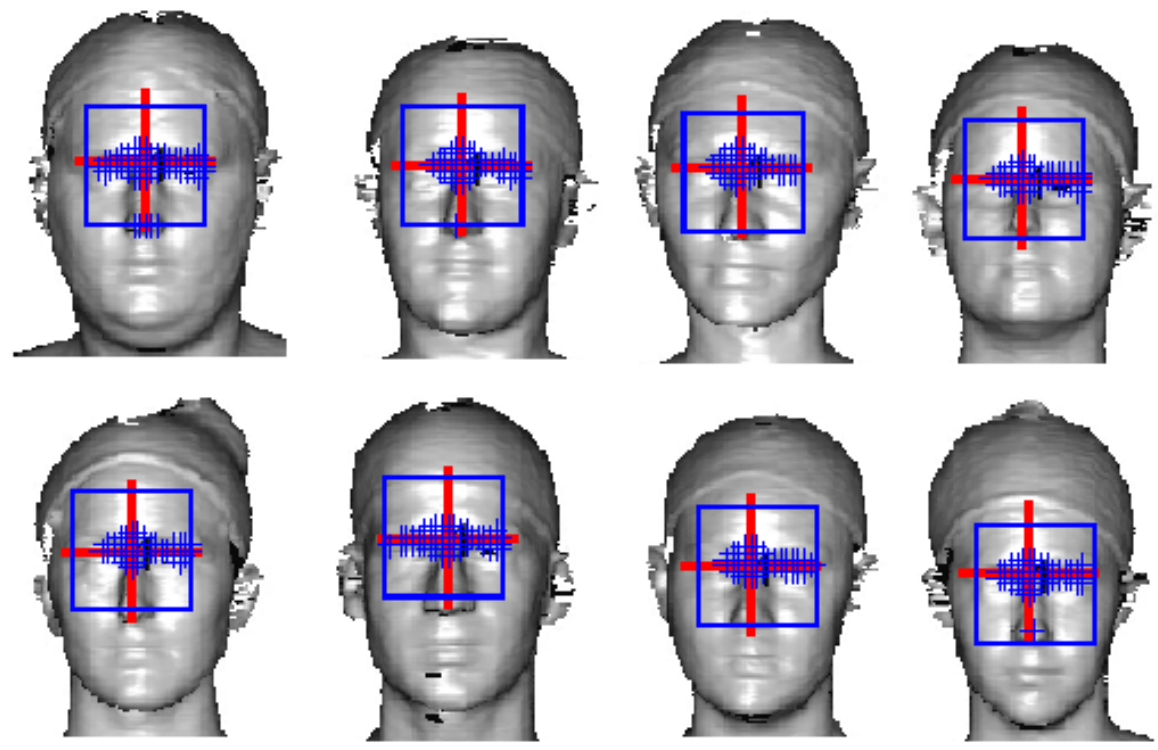

Figure 46: Window-sweeping image patch classification results for Sellion landmark.
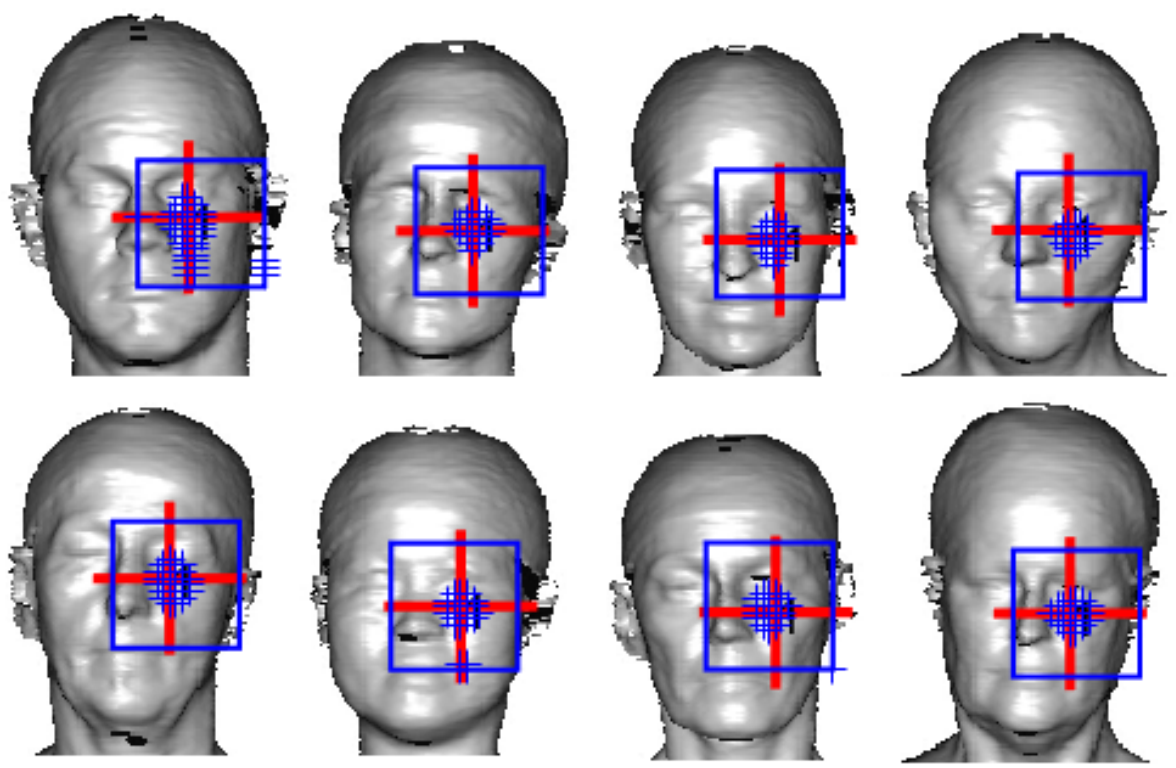

Figure 47: Window-sweeping image patch classification results for Infraorbitale landmark. 

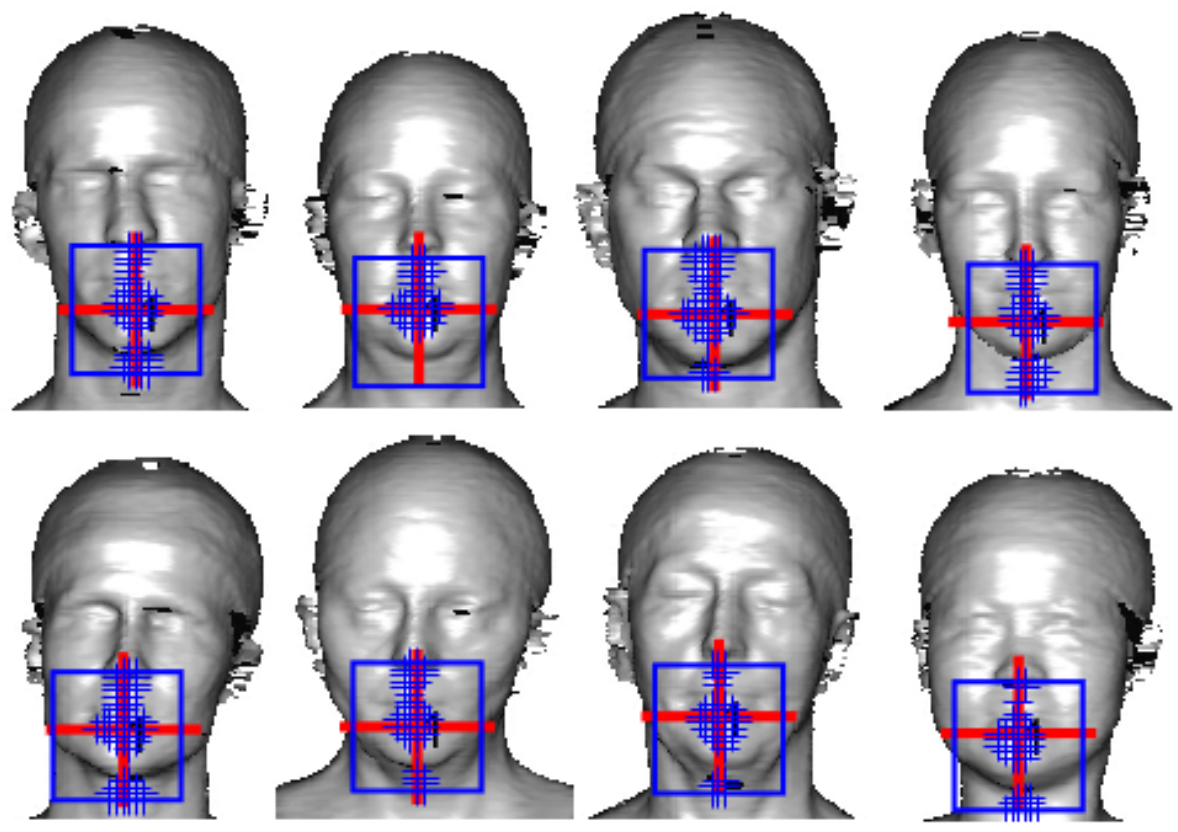

Figure 48: Window-sweeping image patch classification results for Supramenton landmark.

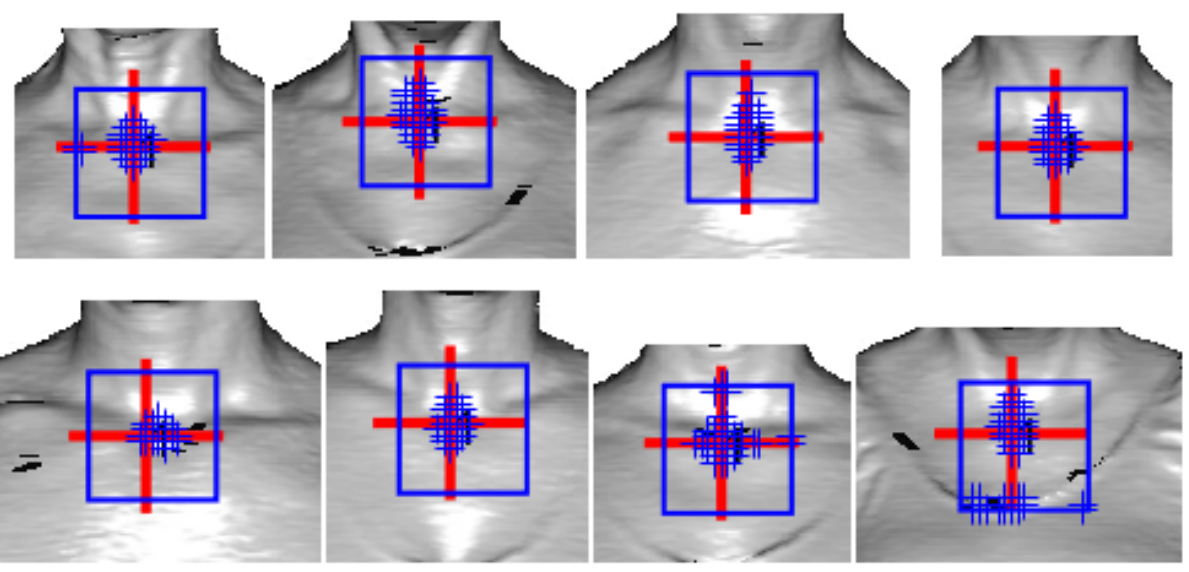

Figure 49: Window-sweeping image patch classification results for Suprasternale landmark. 

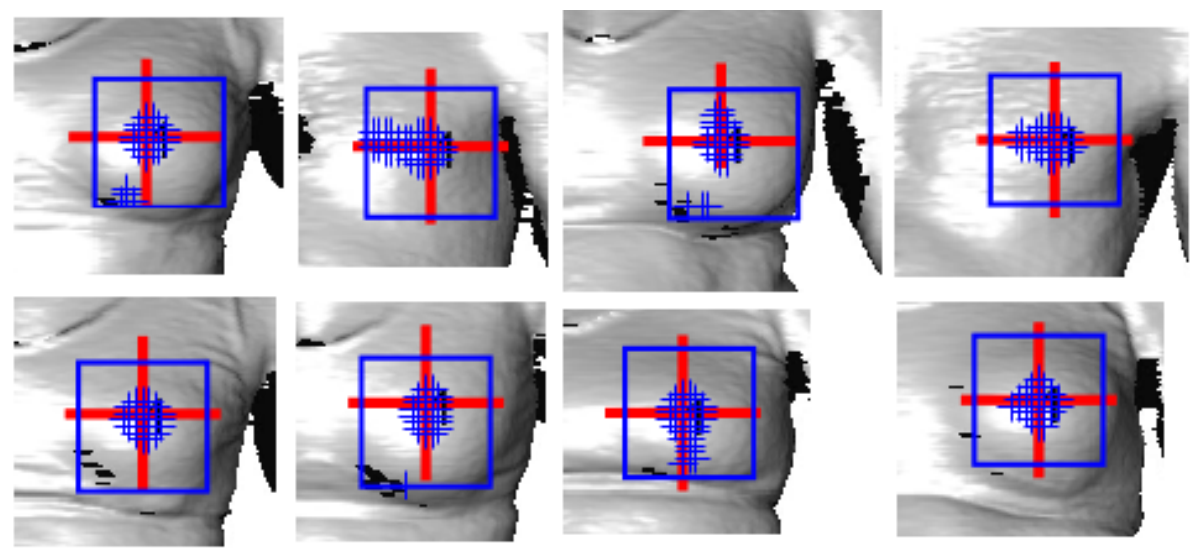

Figure 50: Window-sweeping image patch classification results for Thelion landmark.
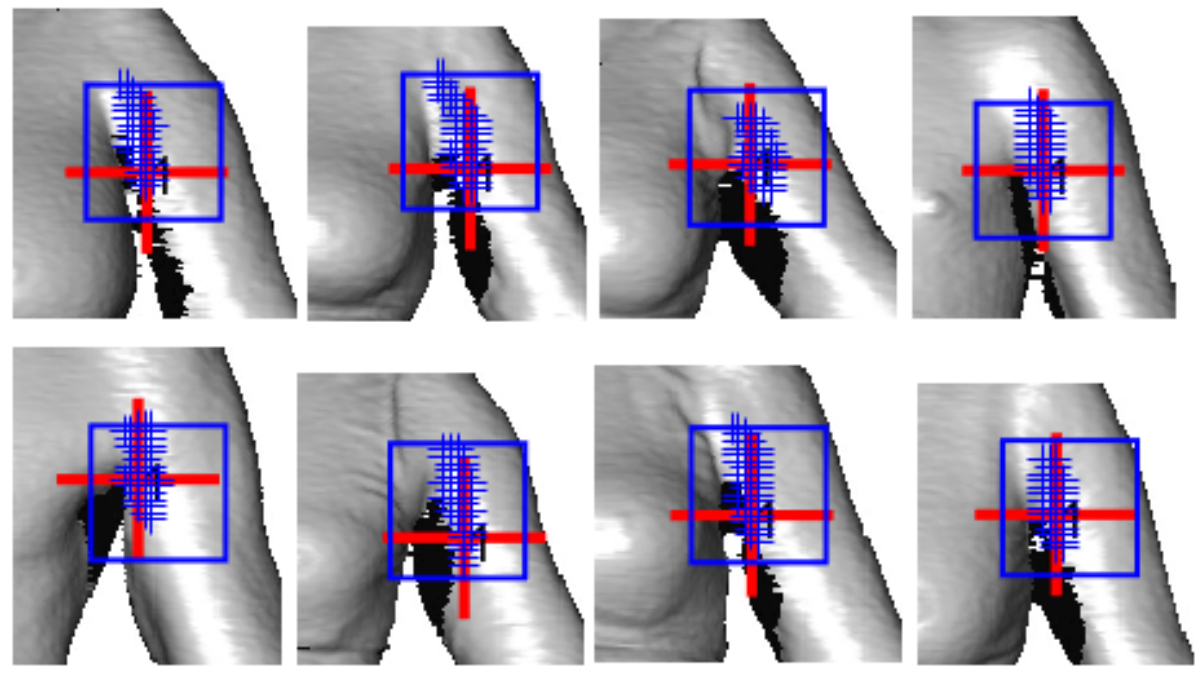

Figure 51: Window-sweeping image patch classification results for Anterior Axilla landmark. 

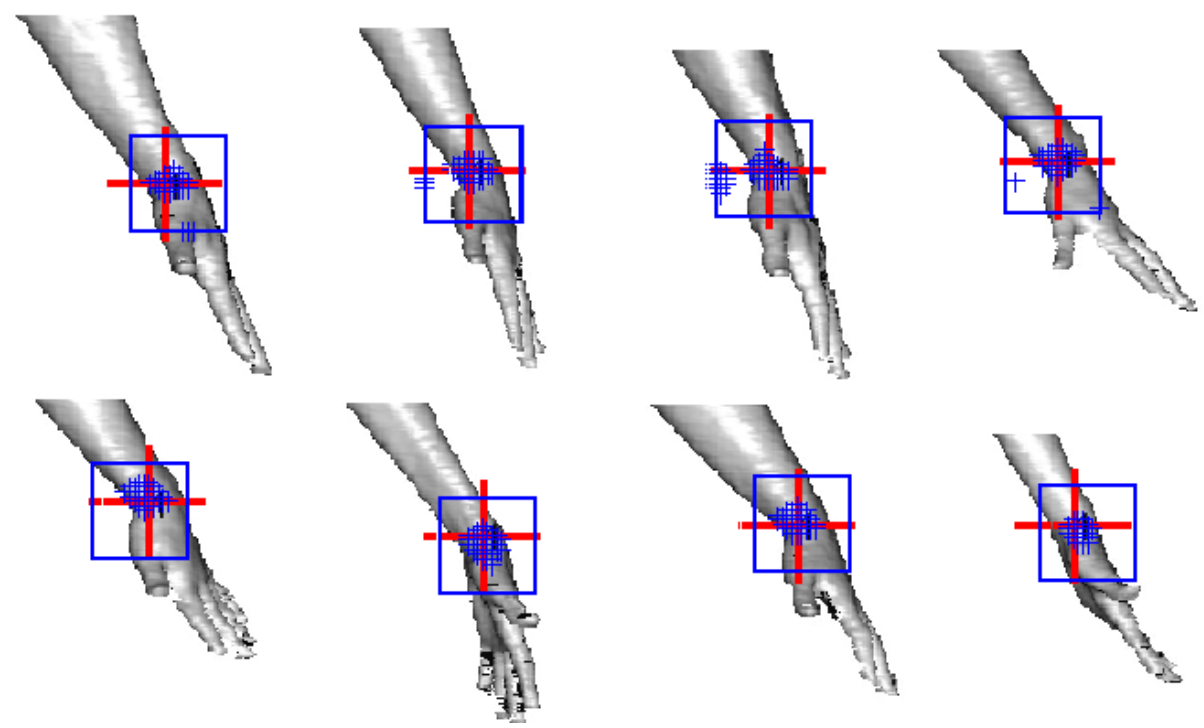

Figure 52: Window-sweeping image patch classification results for Radial Styloid landmark.
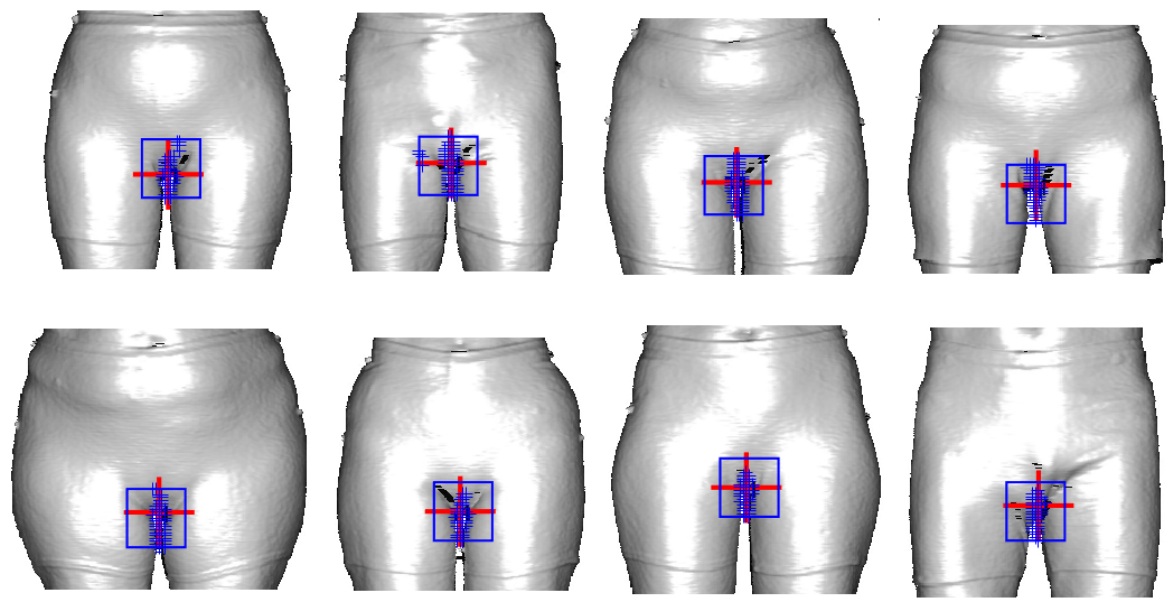

Figure 53: Window-sweeping image patch classification results for Groin landmark. 


\section{Chapter 7}

\section{Conclusion}

Applying deep learning to the analysis of medical imaging data faces challenges; therefore, we have proposed a deep representation learning framework for medical imaging data in one, two or three dimensions. Through a series of validations, the proposed framework proves to outperform traditional approaches by a large margin. Because of its effectiveness, this deep learning framework serves as a guidance in solving other medical data analysis problems.

The proposed framework is composed of data representation and feature learning, both of which are essential for medical imaging data analysis. The different data representations and neural architectures are found to play complementary roles in solving the problems. For example, the data representations used for murmur detection, i.e, spectrogram, scalogram and mel spectrogram, lead to different performance while using the same classification approach. Likewise, appearance, depth and curvature images result in difference accuracy on predicting the location of landmarks. In both applications, the selection of deep architectures also affects the performance. The complementary roles played by the different representations and architectures suggest that multi-modal data fusion approaches will further improve performance on these tasks [139].

The validations on the proposed framework lead to the following summaries on 
choosing proper approaches for medical imaging data analysis. When sufficient training data are available with annotations, training deep neural networks leads to good performance. When the number of training data is reduced, fine-tuning a pre-trained deep neural network becomes a selected approach. When the number of training data is insufficient for fine-tuning a deep neural network, using a pre-trained neural network as off-the-shelf feature extractor normally outperforms manual features or has similar performance.

Representation learning is essential for medical imaging data analysis. Looking forward, we identify several research directions that are promising in further improving the performance of the proposed framework. The first is to use the different variants of generative adversarial networks (GANs) for data augmentation [140]; the second is to use automatic machine learning tools including neural architecture search (NAS) for designing better neural network architectures [141-143]. Petri nets can be tested for modeling complex process behaviors [144]. Last but not least, few-shot learning is also promising when training data with annotations are not enough for training the deep models from scratch $[145,146]$.

The proposed framework can be applied to other data analysis problems, which were tackled using traditional approaches. In 1D signal processing, it can be applied to voice activity detection, speech quality prediction and security monitoring [147-149]. The framework can also be used for determining sit-to-stand transition for home monitoring $[150,151]$. To combat pandemic including COVID-19, the framework has applications in extracting audio features for detecting dry or wet coughs $[152,153]$.

The framework can find its applications in 2D image analysis. The auto-encoder approach can be used for image de-noising [154]. In human modeling, it can be used for localizing spine vertebrae [155], through replacing Active Appearance Models [156]. For health monitoring, it can be applied for measuring pulse transit time through video magnification [157]. 
The framework can be used for 3D data analysis. In particular, it can be used to improve the detection of landmark locations $[158,159]$, which is of high importance for digital human modeling. Despite marker-less approaches [160], the automatic prediction of landmark locations [161] remains an essential step in human modeling, which includes the analysis of human body parts [162-167], posture-invariant shape analysis [168], and shape reconstruction [169-171].

The proposed framework can also find its applications in a pandemic such as the COVID-19. Besides cough detection $[152,153]$, it can be used for computer-aided diagnosis from medical images (X-ray or CT) $[172,173]$ and lowering the risk of infection for medical professionals [174]. 


\section{List of References}

[1] Y. Bengio, A. Courville, and P. Vincent, "Representation learning: A review and new perspectives," IEEE Transactions on Pattern Analysis and Machine Intelligence, vol. 35, pp. 1798-1828, Aug 2013.

[2] O. Russakovsky, J. Deng, H. Su, J. Krause, S. Satheesh, S. Ma, Z. Huang, A. Karpathy, A. Khosla, M. Bernstein, A. C. Berg, and L. Fei-Fei, "ImageNet Large Scale Visual Recognition Challenge," International Journal of Computer Vision (IJCV), vol. 115, no. 3, pp. 211-252, 2015.

[3] D. L. Ripley and T. Politzer, "Vision disturbance after tbi," NeuroRehabilitation, vol. 27, no. 3, p. 215, 2010.

[4] M. Manassi, B. Sayim, and M. H. Herzog, "When crowding of crowding leads to uncrowding," Journal of Vision, vol. 13, pp. 10-10, 112013.

[5] M. D. Zeiler and R. Fergus, Visualizing and Understanding Convolutional Networks, pp. 818-833. Cham: Springer International Publishing, 2014.

[6] A. Krizhevsky, I. Sutskever, and G. E. Hinton, "Imagenet classification with deep convolutional neural networks," in Advances in Neural Information Processing Systems 25 (F. Pereira, C. J. C. Burges, L. Bottou, and K. Q. Weinberger, eds.), pp. 1097-1105, Curran Associates, Inc., 2012.

[7] K. Simonyan and A. Zisserman, "Very deep convolutional networks for largescale image recognition," CoRR, vol. abs/1409.1556, 2014.

[8] C. Szegedy, W. Liu, Y. Jia, P. Sermanet, S. Reed, D. Anguelov, D. Erhan, V. Vanhoucke, and A. Rabinovich, "Going deeper with convolutions," in Computer Vision and Pattern Recognition (CVPR), 2015.

[9] K. He, X. Zhang, S. Ren, and J. Sun, "Deep residual learning for image recognition," CoRR, vol. abs/1512.03385, 2015. 
[10] J. Yosinski, J. Clune, Y. Bengio, and H. Lipson, "How transferable are features in deep neural networks?," in Proceedings of the 27th International Conference on Neural Information Processing Systems - Volume 2, NIPS'14, (Cambridge, MA, USA), pp. 3320-3328, MIT Press, 2014.

[11] R. Tomasello, T. Wennekers, M. Garagnani, and F. Pulvermüller, "Visual cortex recruitment during language processing in blind individuals is explained by hebbian learning," Scientific Reports, vol. 9, no. 1, p. 3579, 2019.

[12] M. J. van Ackeren, F. Barbero, S. Mattioni, R. Bottini, and O. Collignon, "Neuronal populations in the occipital cortex of the blind synchronize to the temporal dynamics of speech," bioRxiv, 2017.

[13] P. Xi, R. Goubran, and C. Shu, "A unified deep learning framework for multimodal multi-dimensional data," in 2019 IEEE International Symposium on Medical Measurements and Applications (MeMeA), (Istanbul, Turkey), June 2019.

[14] P. Xi, R. Goubran, and C. Shu, "Cardiac murmur classification in phonocardiograms using deep convolutional neural networks," in Proceedings of the International Conference on Pattern Recognition and Artificial Intelligence, (Montreal, Quebec), May 2018.

[15] P. Xi, R. Goubran, and C. Shu, Cardiac Murmur Classification in Phonocardiograms using Deep Recurrent-Convolutional Neural Networks, ch. 11, pp. 189210. World Scientific, 2019.

[16] P. Xi, A. Law, R. Goubran, and C. Shu, "Pilot workload prediction from ecg using deep convolutional neural networks," in 2019 IEEE International Symposium on Medical Measurements and Applications (MeMeA), (Istanbul, Turkey), June 2019.

[17] P. Xi, C. Shu, and R. Goubran, "Abnormality detection in mammography using deep convolutional neural networks," in 2018 IEEE International Symposium on Medical Measurements and Applications (MeMeA), (Rome, Italy), June 2018.

[18] P. Xi, H. Guan, C. Shu, L. Borgeat, and R. Goubran, "An integrated approach for medical abnormality detection using deep patch convolutional neural networks," The Visual Computer, 2019.

[19] P. Xi, G. Rouhafzay, H. Guan, C. Shu, L. Borgeat, and R. Goubran, Computer Aided Detection of Abnormality in Mammography using Deep Object Detectors. Elsevier, 2020. 
[20] G. Rouhafzay, Y. Li, H. Guan, C. Shu, R. Goubran, and P. Xi, "An integrated deep approach for lesion detection in breast mri," in Proceedings of Second International Conference on Pattern Recognition and Artificial Intelligence, 2020.

[21] P. Xi, C. Shu, and R. Goubran, "Comparing 2d image features on viewpoint independence using 3d anthropometric dataset," International Journal of the Digital Human, vol. 1, pp. 412-425, 2016.

[22] P. Xi, C. Shu, and R. Goubran, "Localizing 3-d anatomical landmarks using deep convolutional neural networks," in 201714 th Conference on Computer and Robot Vision (CRV), pp. 197-204, May 2017.

[23] P. Smyth, "Clustering sequences with hidden markov models," in Proceedings of the 9th International Conference on Neural Information Processing Systems, NIPS'96, (Cambridge, MA, USA), pp. 648-654, MIT Press, 1996.

[24] "Reinforcement learning." https://en.wikipedia.org/wiki/Reinforcement_learning. Accessed: 2020-05-16.

[25] "Feature learning." https://en.wikipedia.org/wiki/Feature_engineering. Accessed: 2020-05-16.

[26] "Recurrent neural network." https://en.wikipedia.org/wiki/Recurrent_neural_network. Accessed: 2020-05-16.

[27] J. Chung, Ç. Gülçehre, K. Cho, and Y. Bengio, "Empirical evaluation of gated recurrent neural networks on sequence modeling," CoRR, vol. abs/1412.3555, 2014 .

[28] T. Thireou and M. Reczko, "Bidirectional long short-term memory networks for predicting the subcellular localization of eukaryotic proteins," IEEE/ACM Transactions on Computational Biology and Bioinformatics, vol. 4, pp. 441-446, July 2007.

[29] A. Graves and J. Schmidhuber, "Framewise phoneme classification with bidirectional lstm and other neural network architectures," Neural Networks, vol. 18, no. 5, pp. $602-610,2005$. IJCNN 2005.

[30] D. chen He and L. Wang, "Texture unit, texture spectrum, and texture analysis," IEEE Transactions on Geoscience and Remote Sensing, vol. 28, pp. 509512, Jul 1990. 
[31] L. Wang and D.-C. He, "Texture classification using texture spectrum," Pattern Recognition, vol. 23, no. 8, pp. 905 - 910, 1990.

[32] N. Dalal and B. Triggs, "Histograms of oriented gradients for human detection," in 2005 IEEE Computer Society Conference on Computer Vision and Pattern Recognition (CVPR'05), vol. 1, pp. 886-893 vol. 1, June 2005.

[33] A. Coates, H. Lee, and A. Y. Ng, "An analysis of single-layer networks in unsupervised feature learning," in AISTATS, 2011.

[34] G. E. Hinton and R. R. Salakhutdinov, "Reducing the dimensionality of data with neural networks," Science, vol. 313, no. 5786, pp. 504-507, 2006.

[35] J. Masci, U. Meier, D. Cireşan, and J. Schmidhuber, "Stacked convolutional auto-encoders for hierarchical feature extraction," in Proceedings of the 21th International Conference on Artificial Neural Networks - Volume Part I, ICANN'11, (Berlin, Heidelberg), pp. 52-59, Springer-Verlag, 2011.

[36] S. Dasgupta, K. Fang, K. Chen, and S. Savarese, "Delay: Robust spatial layout estimation for cluttered indoor scenes," in The IEEE Conference on Computer Vision and Pattern Recognition (CVPR), June 2016.

[37] J. Long, E. Shelhamer, and T. Darrell, "Fully convolutional networks for semantic segmentation," CoRR, vol. abs/1411.4038, 2014.

[38] Y. B. Yann LeCun and G. Hinton, "Deep learning," Nature, vol. 521, pp. 436444, 2015.

[39] J. Donahue, Y. Jia, O. Vinyals, J. Hoffman, N. Zhang, E. Tzeng, and T. Darrell, "Decaf: A deep convolutional activation feature for generic visual recognition," CoRR, vol. abs/1310.1531, 2013.

[40] M. Oquab, L. Bottou, I. Laptev, and J. Sivic, "Learning and transferring midlevel image representations using convolutional neural networks," in Proceedings of the 2014 IEEE Conference on Computer Vision and Pattern Recognition, CVPR '14, (Washington, DC, USA), pp. 1717-1724, IEEE Computer Society, 2014 .

[41] A. Sharif Razavian, H. Azizpour, J. Sullivan, and S. Carlsson, "Cnn features off-the-shelf: An astounding baseline for recognition," in The IEEE Conference on Computer Vision and Pattern Recognition (CVPR) Workshops, June 2014. 
[42] P. Sermanet, D. Eigen, X. Zhang, M. Mathieu, R. Fergus, and Y. LeCun, "Overfeat: Integrated recognition, localization and detection using convolutional networks," CoRR, vol. abs/1312.6229, 2013.

[43] Y. Jia, E. Shelhamer, J. Donahue, S. Karayev, J. Long, R. Girshick, S. Guadarrama, and T. Darrell, "Caffe: Convolutional architecture for fast feature embedding," arXiv preprint arXiv:1408.5093, 2014.

[44] M. Lin, Q. Chen, and S. Yan, "Network in network," CoRR, vol. abs/1312.4400, 2013.

[45] F. Chollet, "Xception: Deep learning with depthwise separable convolutions," in 2017 IEEE Conference on Computer Vision and Pattern Recognition (CVPR), pp. 1800-1807, July 2017.

[46] C. Szegedy, S. Ioffe, V. Vanhoucke, and A. A. Alemi, "Inception-v4, inceptionresnet and the impact of residual connections on learning," in Thirty-First AAAI Conference on Artificial Intelligence, 2017.

[47] S. Xie, R. Girshick, P. Dollár, Z. Tu, and K. He, "Aggregated residual transformations for deep neural networks," in Proceedings of the IEEE conference on computer vision and pattern recognition, pp. 1492-1500, 2017.

[48] "World statistics on cardiovascular disease." www.who.int/mediacentre/factsheets/fs317/en/. Accessed: 2020-05-16.

[49] S. Sanei, M. Ghodsi, and H. Hassani, "An adaptive singular spectrum analysis approach to murmur detection from heart sounds," Medical Engineering \&6 Physics, vol. 33, no. 3, pp. 362 - 367, 2011.

[50] F. Safara, S. Doraisamy, A. Azman, A. Jantan, and S. Ranga, "Wavelet packet entropy for heart murmurs classification," Advances in Bioinformatics, vol. 2012, 2012.

[51] A. T. Balogh, Analysis of the Heart Sounds and Murmurs of Fetuses and Preterm Infants. PhD thesis, Pazmany Peter Catholic University, 2012.

[52] C. Liu, D. Springer, Q. Li, B. Moody, R. A. Juan, F. J. Chorro, F. Castells, J. M. Roig, I. Silva, A. E. W. Johnson, Z. Syed, S. E. Schmidt, C. D. Papadaniil, L. Hadjileontiadis, H. Naseri, A. Moukadem, A. Dieterlen, C. Brandt, H. Tang, M. Samieinasab, M. R. Samieinasab, R. Sameni, R. G. Mark, and G. D. Clifford, "An open access database for the evaluation of heart sound algorithms," Physiological Measurement, vol. 37, no. 12, p. 2181, 2016. 
[53] C. Potes, S. Parvaneh, A. Rahman, and B. Conroy, "Ensemble of feature-based and deep learning-based classifiers for detection of abnormal heart sounds," in 2016 Computing in Cardiology Conference (CinC), pp. 621-624, Sept 2016.

[54] M. Zabihi, A. B. Rad, S. Kiranyaz, M. Gabbouj, and A. K. Katsaggelos, "Heart sound anomaly and quality detection using ensemble of neural networks without segmentation," in 2016 Computing in Cardiology Conference (CinC), pp. 613616, Sept 2016.

[55] E. Kay and A. Agarwal, "Dropconnected neural network trained with diverse features for classifying heart sounds," in 2016 Computing in Cardiology Conference (CinC), pp. 617-620, Sept 2016.

[56] "The effectiveness of data augmentation in image classification using deep learning." http://cs231n.stanford.edu/reports/2017/pdfs/300.pdf. Accessed: 202005-16.

[57] N. Srivastava, G. Hinton, A. Krizhevsky, I. Sutskever, and R. Salakhutdinov, "Dropout: A simple way to prevent neural networks from overfitting," Journal of Machine Learning Research, vol. 15, pp. 1929-1958, 2014.

[58] S. Ioffe and C. Szegedy, "Batch normalization: Accelerating deep network training by reducing internal covariate shift," CoRR, vol. abs/1502.03167, 2015.

[59] Z. Huang, Z. Pan, and B. Lei, "Transfer learning with deep convolutional neural network for sar target classification with limited labeled data," Remote Sensing, vol. 9, no. 9, 2017.

[60] P. Thodoroff, J. Pineau, and A. Lim, "Learning robust features using deep learning for automatic seizure detection," CoRR, vol. abs/1608.00220, 2016.

[61] P. Bashivan, I. Rish, M. Yeasin, and N. Codella, "Learning representations from EEG with deep recurrent-convolutional neural networks," CoRR, vol. abs/1511.06448, 2015.

[62] P. Bentley, G. Nordehn, M. Coimbra, and S. Mannor, "The PASCAL Classifying Heart Sounds Challenge 2011 (CHSC2011) Results." http://www.peterjbentley.com/heartchallenge/index.html.

[63] N. Ranganathan, V. Sivaciyan, and F. Saksena, The Art and Science of Cardiac Physical Examination: With Heart Sounds and Pulse Wave Forms on CD. Contemporary Cardiology, Humana Press, 2007. 
[64] S. Hochreiter and J. Schmidhuber, "Long short-term memory," Neural Computation, vol. 9, no. 8, pp. 1735-1780, 1997.

[65] E. F. Gomes and E. Pereira, "Classifying heart sounds using peak location for segmentation and feature construction." http://www.peterjbentley.com/heartworkshop/challengepaper1.pdf.

[66] Y. Deng and P. J. Bentley, "A robust heart sound segmentation and classification algorithm using wavelet decomposition and spectrogram." http://www.peterjbentley.com/heartworkshop/challengepaper3.pdf.

[67] A. Chao, S. Ng, and L. Wang, "Listen to your heart: Feature extraction and classification methods for heart sounds." https://lindawangg.github.io/projects/classifying-heartbeats.pdf.

[68] L. van der Maaten and G. Hinton, "Visualizing data using t-SNE," Journal of Machine Learning Research, vol. 9, pp. 2579-2605, 2008.

[69] P. L. A. Hernández, T. T. Estrada, A. L. Pizarro, M. L. D. Cisternas, and C. S. Tapia, "Calcificaciones mamarias: descripción y clasificación según la 5.a edición bi-rads," Revista Chilena de Radiología, vol. 22, no. 2, pp. 80 - 91, 2016.

[70] D. Ciresan, A. Giusti, L. M. Gambardella, and J. Schmidhuber, "Deep neural networks segment neuronal membranes in electron microscopy images," in Advances in Neural Information Processing Systems 25 (F. Pereira, C. J. C. Burges, L. Bottou, and K. Q. Weinberger, eds.), pp. 2843-2851, Curran Associates, Inc., 2012.

[71] T. Kooi, G. Litjens, B. van Ginneken, A. Gubern-Mérida, C. I. Sánchez, R. Mann, A. den Heeten, and N. Karssemeijer, "Large scale deep learning for computer aided detection of mammographic lesions," Medical Image Analysis, vol. 35, pp. $303-312,2017$.

[72] S. Ren, K. He, R. Girshick, and J. Sun, "Faster r-cnn: Towards real-time object detection with region proposal networks," IEEE Transactions on Pattern Analysis \& Machine Intelligence, vol. 39, pp. 1137-1149, June 2017.

[73] M. A. Mazurowski, M. Buda, A. Saha, and M. R. Bashir, "Deep learning in radiology: an overview of the concepts and a survey of the state of the art," CoRR, vol. abs/1802.08717, 2018. 
[74] X. Wang, Y. Peng, L. Lu, Z. Lu, M. Bagheri, and R. M. Summers, "Chestx-ray8: Hospital-scale chest x-ray database and benchmarks on weaklysupervised classification and localization of common thorax diseases," CoRR, vol. abs/1705.02315, 2017.

[75] P. Rajpurkar, J. Irvin, K. Zhu, B. Yang, H. Mehta, T. Duan, D. Ding, A. Bagul, C. Langlotz, K. Shpanskaya, M. P. Lungren, and A. Y. Ng, "Chexnet: Radiologist-level pneumonia detection on chest x-rays with deep learning," CoRR, vol. abs/1711.05225, 2017.

[76] P. Rajpurkar, J. Irvin, A. Bagul, D. Ding, T. Duan, H. Mehta, B. Yang, K. Zhu, D. Laird, R. L. Ball, C. Langlotz, K. Shpanskaya, M. P. Lungren, and A. Ng, "Mura dataset: Towards radiologist-level abnormality detection in musculoskeletal radiographs," arXiv, 2017.

[77] B. Zhou, A. Khosla, L. A., A. Oliva, and A. Torralba, "Learning Deep Features for Discriminative Localization.," CVPR, 2016.

[78] R. S. Lee, F. Gimenez, A. Hoogi, K. K. Miyake, M. Gorovoy, and D. L. Rubin, "A curated mammography data set for use in computer-aided detection and diagnosis research," Scientific Data, vol. 4:170177, 2017/12/19/online.

[79] R. Nakayama, Y. Uchiyama, K. Yamamoto, R. Watanabe, and K. Namba, "Computer-aided diagnosis scheme using a filter bank for detection of microcalcification clusters in mammograms," IEEE Transactions on Biomedical Engineering, vol. 53, pp. 273-283, Feb 2006.

[80] K. Kavitha and N. Kumaravel, "A comparitive study of various microcalcification cluster detection methods in digitized mammograms," in 2007 14th International Workshop on Systems, Signals and Image Processing and 6th EURASIP Conference focused on Speech and Image Processing, Multimedia Communications and Services, pp. 405-409, June 2007.

[81] N. R. Pal, B. Bhowmick, S. K. Patel, S. Pal, and J. Das, "A multi-stage neural network aided system for detection of microcalcifications in digitized mammograms," Neurocomputing, vol. 71, no. 13, pp. 2625 - 2634, 2008. Artificial Neural Networks (ICANN 2006) / Engineering of Intelligent Systems (ICEIS 2006).

[82] F. Harirchi, P. Radparvar, H. A. Moghaddam, F. Dehghan, and M. Giti, "Twolevel algorithm for mcs detection in mammograms using diverse-adaboost-svm," in 2010 20th International Conference on Pattern Recognition, pp. 269-272, Aug 2010 . 
[83] A. Oliver, A. Torrent, X. Lladó, M. Tortajada, L. Tortajada, M. Sentís, J. Freixenet, and R. Zwiggelaar, "Automatic microcalcification and cluster detection for digital and digitised mammograms," Know.-Based Syst., vol. 28, pp. 68-75, Apr. 2012.

[84] X. Zhang and X. Gao, "Twin support vector machines and subspace learning methods for microcalcification clusters detection," Engineering Applications of Artificial Intelligence, vol. 25, no. 5, pp. 1062 - 1072, 2012.

[85] "Acr bi-rads-mammography ultrasound and magnetic resonance imaging," 4th ed., American College of Radiology, 2003.

[86] Y. Li, H. Chen, L. Cao, and J. Ma, "A survey of computer-aided detection of breast cancer with mammography," J Health Med Informat, vol. 7, no. 238, 2016 .

[87] A. Petrosian, H.-P. Chan, M. A. Helvie, M. M. Goodsitt, and D. D. Adler, "Computer-aided diagnosis in mammography: classification of mass and normal tissue by texture analysis," Physics in Medicine and Biology, vol. 39, no. 12, pp. 2273-2288, 1994.

[88] N. Petrick, H.-P. Chan, B. Sahiner, and D. Wei, "An adaptive density-weighted contrast enhancement filter for mammographic breast mass detection," IEEE Transactions on Medical Imaging, vol. 15, pp. 59-67, Feb 1996.

[89] D. Cascio, F. Fauci, R. Magro, G. Raso, R. Bellotti, F. D. Carlo, S. Tangaro, G. D. Nunzio, M. Quarta, G. Forni, A. Lauria, M. E. Fantacci, A. Retico, G. L. Masala, P. Oliva, S. Bagnasco, S. C. Cheran, and E. L. Torres, "Mammogram segmentation by contour searching and mass lesions classification with neural network," IEEE Transactions on Nuclear Science, vol. 53, pp. 2827-2833, Oct 2006.

[90] S. Han, H.-K. Kang, J.-Y. Jeong, M.-H. Park, W. Kim, W.-C. Bang, and Y.-K. Seong, "A deep learning framework for supporting the classification of breast lesions in ultrasound images," Physics in Medicine $\&$ Biology, vol. 62, no. 19, p. $7714,2017$.

[91] M. H. Yap, G. Pons, J. Martí, S. Ganau, M. Sentís, R. Zwiggelaar, A. K. Davison, and R. Martí, "Automated breast ultrasound lesions detection using convolutional neural networks," IEEE Journal of Biomedical and Health Informatics, vol. 22, pp. 1218-1226, July 2018. 
[92] H. Li, J. Weng, Y. Shi, W. Gu, Y. Mao, Y. Wang, W. Liu, and J. Zhang, "An improved deep learning approach for detection of thyroid papillary cancer in ultrasound images," Scientific Reports, vol. 8, no. 1, p. 6600, 2018.

[93] J. Chi, E. Walia, P. Babyn, J. Wang, G. Groot, and M. Eramian, "Thyroid nodule classification in ultrasound images by fine-tuning deep convolutional neural network," Journal of Digital Imaging, vol. 30, pp. 477-486, Aug 2017.

[94] H. Fu, J. Cheng, Y. Xu, D. W. K. Wong, J. Liu, and X. Cao, “Joint optic disc and cup segmentation based on multi-label deep network and polar transformation," CoRR, vol. abs/1801.00926, 2018.

[95] H. Fu, Y. Xu, S. Lin, D. W. K. Wong, and J. Liu, "Deepvessel: Retinal vessel segmentation via deep learning and conditional random field," in International conference on medical image computing and computer-assisted intervention, pp. 132-139, Springer, 2016.

[96] P. F. Felzenszwalb, R. B. Girshick, D. McAllester, and D. Ramanan, "Object detection with discriminatively trained part-based models," IEEE Transactions on Pattern Analysis and Machine Intelligence, vol. 32, pp. 1627-1645, Sep. 2010.

[97] R. Girshick, J. Donahue, T. Darrell, and J. Malik, "Rich feature hierarchies for accurate object detection and semantic segmentation," in Proceedings of the 2014 IEEE Conference on Computer Vision and Pattern Recognition, CVPR '14, (Washington, DC, USA), pp. 580-587, IEEE Computer Society, 2014.

[98] R. Sa, W. Owens, R. Wiegand, and V. Chaudhary, "Fast scale-invariant lateral lumbar vertebrae detection and segmentation in x-ray images," in 2016 38th Annual International Conference of the IEEE Engineering in Medicine and Biology Society (EMBC), pp. 1054-1057, Aug 2016.

[99] R. B. Girshick, "Fast R-CNN," CoRR, vol. abs/1504.08083, 2015.

[100] W. Liu, D. Anguelov, D. Erhan, C. Szegedy, S. Reed, C.-Y. Fu, and A. C. Berg, "Ssd: Single shot multibox detector," in Computer Vision - ECCV 2016 (B. Leibe, J. Matas, N. Sebe, and M. Welling, eds.), (Cham), pp. 2137, Springer International Publishing, 2016.

[101] J. Dai, Y. Li, K. He, and J. Sun, "R-fcn: Object detection via region-based fully convolutional networks," in Advances in Neural Information Processing Systems 29 (D. D. Lee, M. Sugiyama, U. V. Luxburg, I. Guyon, and R. Garnett, eds.), pp. 379-387, Curran Associates, Inc., 2016. 
[102] J. Huang, V. Rathod, C. Sun, M. Zhu, A. Korattikara, A. Fathi, I. Fischer, Z. Wojna, Y. Song, S. Guadarrama, and K. Murphy, "Speed/accuracy tradeoffs for modern convolutional object detectors," in 2017 IEEE Conference on Computer Vision and Pattern Recognition (CVPR), pp. 3296-3297, July 2017.

[103] J. Redmon, S. K. Divvala, R. B. Girshick, and A. Farhadi, "You only look once: Unified, real-time object detection," CoRR, vol. abs/1506.02640, 2015.

[104] J. Redmon and A. Farhadi, "Yolo9000: better, faster, stronger," in Proceedings of the IEEE conference on computer vision and pattern recognition, pp. 7263$7271,2017$.

[105] J. S. et al, "The mammographic image analysis society digital mammogram database," Exerpta Medica. International Congress Series, vol. 1069, pp. 375378, 1994.

[106] M. Heath, K. Bowyer, D. Kopans, R. Moore, and W. P. Kegelmeyer, "The digital database for screening mammography," in Proceedings of the Fifth International Workshop on Digital Mammography, pp. 212-218, 2001.

[107] C. Papageorgiou and T. Poggio, "A trainable system for object detection," International Journal of Computer Vision, vol. 38, no. 1, pp. 15-33, 2000.

[108] D. G. Lowe, "Object recognition from local scale-invariant features," in Computer Vision, 1999. The Proceedings of the Seventh IEEE International Conference on, vol. 2, pp. 1150-1157 vol.2, 1999.

[109] S. Belongie and J. Malik, "Matching with shape contexts," in Content-based Access of Image and Video Libraries, 2000. Proceedings. IEEE Workshop on, pp. 20-26, 2000.

[110] X. Wang, T. X. Han, and S. Yan, "An hog-lbp human detector with partial occlusion handling," in 2009 IEEE 12th International Conference on Computer Vision, pp. 32-39, Sept 2009.

[111] Z. Wu, S. Pan, F. Chen, G. Long, C. Zhang, and S. Y. Philip, "A comprehensive survey on graph neural networks," IEEE Transactions on Neural Networks and Learning Systems, 2020.

[112] M. M. Bronstein, J. Bruna, Y. LeCun, A. Szlam, and P. Vandergheynst, "Geometric deep learning: going beyond euclidean data," IEEE Signal Processing Magazine, vol. 34, no. 4, pp. 18-42, 2017. 
[113] K. M. Robinette, H. Daanen, and E. Paquet, "The caesar project: a 3-d surface anthropometry survey," in Proceedings of Second International Conference on 3-D Digital Imaging and Modeling, pp. 380-386, 1999.

[114] C.-W. Hsu and C.-J. Lin, "A comparison of methods for multiclass support vector machines," Trans. Neur. Netw., vol. 13, pp. 415-425, Mar. 2002.

[115] Y. Lecun, L. Bottou, Y. Bengio, and P. Haffner, "Gradient-based learning applied to document recognition," Proceedings of the IEEE, vol. 86, pp. 2278-2324, Nov 1998.

[116] H. Lee, R. Grosse, R. Ranganath, and A. Y. Ng, "Convolutional deep belief networks for scalable unsupervised learning of hierarchical representations," in Proceedings of the 26th International Conference on Machine Learning, ICML '09, (New York, NY, USA), pp. 609-616, ACM, 2009.

[117] S. Wan, M. W. Mak, and S. Y. Kung, "Adaptive thresholding for multi-label svm classification with application to protein subcellular localization prediction," in 2013 IEEE International Conference on Acoustics, Speech and Signal Processing, pp. 3547-3551, May 2013.

[118] D. Boscaini, J. Masci, S. Melzi, M. M. Bronstein, U. Castellani, and P. Vandergheynst, "Learning class-specific descriptors for deformable shapes using localized spectral convolutional networks," Computer Graphics Forum, vol. 34, no. 5, pp. 13-23, 2015.

[119] J. Masci, D. Boscaini, M. M. Bronstein, and P. Vandergheynst, "Shapenet: Convolutional neural networks on non-euclidean manifolds," CoRR, vol. abs/1501.06297, 2015.

[120] A. Giachetti, E. Mazzi, F. Piscitelli, M. Aono, A. B. Hamza, T. Bonis, P. Claes, A. Godil, C. Li, M. Ovsjanikov, V. Patraucean, C. Shu, J. Snyders, P. Suetens, A. Tatsuma, D. Vandermeulen, S. Wuhrer, and P. Xi, "Automatic location of landmarks used in manual anthropometry," in Eurographics Workshop on 3D Object Retrieval, Strasbourg, France, 2014. Proceedings, pp. 93-100, 2014.

[121] B. Allen, B. Curless, and Z. Popović, "The space of human body shapes: Reconstruction and parameterization from range scans," in ACM SIGGRAPH 2003 Papers, SIGGRAPH '03, (New York, NY, USA), pp. 587-594, ACM, 2003.

[122] P. Xi, W.-S. Lee, and C. Shu, "Analysis of segmented human body scans," in Proceedings of Graphics Interface 200\%, GI '07, (New York, NY, USA), pp. 1926, ACM, 2007. 
[123] B. Allen, B. Curless, and Z. Popovic,, "Exploring the space of human body shapes: Data-driven synthesis under anthropometric control," in SAE Technical Paper, SAE International, 062004.

[124] J. Boisvert, C. Shu, S. Wuhrer, and P. Xi, "Three-dimensional human shape inference from silhouettes: reconstruction and validation," Machine Vision and Applications, vol. 24, no. 1, pp. 145-157, 2013.

[125] S. Zuffi and M. J. Black, "The stitched puppet: A graphical model of 3d human shape and pose," in 2015 IEEE Conference on Computer Vision and Pattern Recognition (CVPR), pp. 3537-3546, June 2015.

[126] C. Szegedy, W. Liu, Y. Jia, P. Sermanet, S. E. Reed, D. Anguelov, D. Erhan, V. Vanhoucke, and A. Rabinovich, "Going deeper with convolutions," CoRR, vol. abs/1409.4842, 2014.

[127] L. Wei, Q. Huang, D. Ceylan, E. Vouga, and H. Li, "Dense human body correspondences using convolutional networks," in Computer Vision and Pattern Recognition (CVPR), 2016.

[128] S. Bu, P. Han, Z. Liu, J. Han, and H. Lin, "Local deep feature learning framework for 3d shape," Computers and Graphics, vol. 46, pp. 117 - 129, 2015. Shape Modeling International 2014.

[129] R. Girshick, J. Donahue, T. Darrell, and J. Malik, "Region-based convolutional networks for accurate object detection and segmentation," IEEE Transactions on Pattern Analysis and Machine Intelligence, vol. 38, pp. 142-158, Jan 2016.

[130] S. Ren, K. He, R. Girshick, and J. Sun, "Faster R-CNN: Towards real-time object detection with region proposal networks," arXiv preprint arXiv:1506.01497, 2015 .

[131] S. Li, Z.-Q. Liu, and A. B. Chan, "Heterogeneous multi-task learning for human pose estimation with deep convolutional neural network," International Journal of Computer Vision, vol. 113, no. 1, pp. 19-36, 2015.

[132] J. Shotton, A. Fitzgibbon, A. Blake, A. Kipman, M. Finocchio, R. Moore, and T. Sharp, "Real-time human pose recognition in parts from a single depth image," in CVPR, IEEE, June 2011.

[133] Z. B. Azouz, C. Shu, and A. Mantel, "Automatic locating of anthropometric landmarks on 3d human models," in 3D Data Processing, Visualization, and Transmission, Third International Symposium on, pp. 750-757, June 2006. 
[134] S. Wuhrer, Z. B. Azouz, and C. Shu, "Posture invariant surface description and feature extraction," in Computer Vision and Pattern Recognition (CVPR), 2010 IEEE Conference on, pp. 374-381, June 2010.

[135] E. Vezzetti and F. Marcolin, "3d human face description: landmarks measures and geometrical features," Image and Vision Computing, vol. 30, no. 10, pp. 698 - 712, 2012. 3D Facial Behaviour Analysis and Understanding.

[136] H. Fan and E. Zhou, "Approaching human level facial landmark localization by deep learning," Image and Vision Computing, vol. 47, pp. 27 - 35, 2016. 300-W, the First Automatic Facial Landmark Detection in-the-Wild Challenge.

[137] K. Chatfield, K. Simonyan, A. Vedaldi, and A. Zisserman, "Return of the devil in the details: Delving deep into convolutional nets," in British Machine Vision Conference, 2014.

[138] A. Vedaldi and K. Lenc, "Matconvnet - convolutional neural networks for matlab," in Proceeding of the ACM Int. Conf. on Multimedia, 2015.

[139] K. Liu, Y. Li, N. Xu, and P. Natarajan, "Learn to combine modalities in multimodal deep learning," arXiv preprint arXiv:1805.11730, 2018.

[140] X. Yi, E. Walia, and P. Babyn, "Generative adversarial network in medical imaging: A review," Medical image analysis, p. 101552, 2019.

[141] T. Elsken, J. H. Metzen, and F. Hutter, "Neural architecture search: A survey," arXiv preprint arXiv:1808.05377, 2018.

[142] H. Liu, K. Simonyan, and Y. Yang, "DARTS: differentiable architecture search," CoRR, vol. abs/1806.09055, 2018.

[143] A. Wong, Z. Qiu Lin, and B. Chwyl, "Attonets: Compact and efficient deep neural networks for the edge via human-machine collaborative design," in Proceedings of the IEEE Conference on Computer Vision and Pattern Recognition Workshops, pp. 0-0, 2019.

[144] J. Theis and H. Darabi, "Dream-nap: Decay replay mining to predict next process activities," arXiv preprint arXiv:1903.05084, 2019.

[145] Y. Wang, Q. Yao, J. Kwok, and L. M. Ni, "Generalizing from a few examples: A survey on few-shot learning," arXiv: 1904.05046, 2019. 
[146] D. Chen, Y. Chen, Y. Li, F. Mao, Y. He, and H. Xue, "Self-supervised learning for few-shot image classification," arXiv preprint arXiv:1911.06045, 2019.

[147] E. Nemer, R. Goubran, and S. Mahmoud, "Robust voice activity detection using higher-order statistics in the lpc residual domain," IEEE Transactions on Speech and Audio Processing, vol. 9, no. 3, pp. 217-231, 2001.

[148] L. Ding and R. A. Goubran, "Speech quality prediction in voip using the extended e-model," in GLOBECOM'03. IEEE Global Telecommunications Conference (IEEE Cat. No. 03CH37489), vol. 7, pp. 3974-3978, IEEE, 2003.

[149] A. R. Abu-El-Quran, R. A. Goubran, and A. D. Chan, "Security monitoring using microphone arrays and audio classification," IEEE Transactions on Instrumentation and Measurement, vol. 55, no. 4, pp. 1025-1032, 2006.

[150] A. Arcelus, M. H. Jones, R. Goubran, and F. Knoefel, "Integration of smart home technologies in a health monitoring system for the elderly," in 21st International Conference on Advanced Information Networking and Applications Workshops (AINAW'Or), vol. 2, pp. 820-825, IEEE, 2007.

[151] A. Arcelus, C. L. Herry, R. A. Goubran, F. Knoefel, H. Sveistrup, and M. Bilodeau, "Determination of sit-to-stand transfer duration using bed and floor pressure sequences," IEEE transactions on biomedical engineering, vol. 56, no. 10, pp. 2485-2492, 2009.

[152] A. R. Abu-El-Quran and R. A. Goubran, "Pitch-based feature extraction for audio classification," in The 2nd IEEE Internatioal Workshop on Haptic, Audio and Visual Environments and Their Applications, 2003. HAVE 2003. Proceedings., pp. 43-47, IEEE, 2003.

[153] H. Chatrzarrin, A. Arcelus, R. Goubran, and F. Knoefel, "Feature extraction for the differentiation of dry and wet cough sounds," in 2011 IEEE International Symposium on Medical Measurements and Applications, pp. 162-166, IEEE, 2011.

[154] P. Xi and T. Xu, "De-noising and recovering images based on kernel pca theory," in The 12-th International Conference in Central Europe on Computer Graphics, Visualization and Computer Vision, pp. 197-200, UNION Agency, 2004 .

[155] P. Xi, T. Xu, and Z. Zhao, "Knowledge-based active appearance model applied in medical image localization," in IEEE International Conference Mechatronics and Automation, 2005, vol. 2, pp. 637-642, IEEE, 2005. 
[156] P. Xi, W.-S. Lee, G. Frederico, C. Joslin, and L. Zhou, "Comprehending and transferring facial expressions based on statistical shape and texture models," in Computer Graphics International Conference, pp. 265-276, Springer, 2006.

[157] X. He, R. A. Goubran, and X. P. Liu, "Using eulerian video magnification framework to measure pulse transit time," in 2014 IEEE International Symposium on Medical Measurements and Applications (MeMeA), pp. 1-4, IEEE, 2014.

[158] C. Herry, M. Frize, and R. A. Goubran, "Segmentation and landmark identification in infrared images of the human body," in 2006 International Conference of the IEEE Engineering in Medicine and Biology Society, pp. 957-960, IEEE, 2006.

[159] A. Giachetti, E. Mazzi, F. Piscitelli, M. Aono, A. B. Hamza, T. Bonis, P. Claes, A. Godil, C. Li, M. Ovsjanikov, et al., "Shrec'14 track: automatic location of landmarks used in manual anthropometry," in Eurographics Workshop on 3D Object Retrieval, 2014.

[160] S. Wuhrer, C. Shu, and P. Xi, "Landmark-free posture invariant human shape correspondence," The Visual Computer, vol. 27, no. 9, pp. 843-852, 2011.

[161] S. Wuhrer, P. Xi, and C. Shu, "Human shape correspondence with automatically predicted landmarks," Machine Vision and Applications, vol. 23, no. 4, pp. 821$830,2012$.

[162] P. Xi, W.-S. Lee, and C. Shu, "Analysis of segmented human body scans," in Proceedings of graphics interface 200\%, pp. 19-26, 2007.

[163] P. Xi, C. Shu, and M. Rioux, "Principal components analysis of 3-d scanned human heads," in ACM SIGGRAPH 2007 posters, pp. 30-es, 2007.

[164] P. Xi and C. Shu, "Consistent parameterization and statistical analysis of human head scans," The visual computer, vol. 25, no. 9, pp. 863-871, 2009.

[165] R. Ball, C. Shu, P. Xi, M. Rioux, Y. Luximon, and J. Molenbroek, "A comparison between chinese and caucasian head shapes," Applied ergonomics, vol. 41, no. 6, pp. 832-839, 2010.

[166] Z. Zhuang, C. Shu, P. Xi, M. Bergman, and M. Joseph, "Head-and-face shape variations of us civilian workers," Applied ergonomics, vol. 44, no. 5, pp. 775$784,2013$. 
[167] Y. Liu, P. Xi, M. Joseph, Z. Zhuang, C. Shu, L. Jiang, M. Bergman, and W. Chen, "Variations in head-and-face shape of chinese civilian workers," Annals of Occupational Hygiene, vol. 59, no. 7, pp. 932-944, 2015.

[168] S. Wuhrer, C. Shu, and P. Xi, "Posture-invariant statistical shape analysis using laplace operator," Computers \& Graphics, vol. 36, no. 5, pp. 410-416, 2012.

[169] P. Xi, "A pca-based approach to the three-dimensional reconstruction of human body from single frontal-view silhouette," Master's thesis, University of Ottawa (Canada), 2007.

[170] P. Xi, W.-S. Lee, and C. Shu, "A data-driven approach to human-body cloning using a segmented body database," in 15th Pacific Conference on Computer Graphics and Applications (PG'0\%), pp. 139-147, IEEE, 2007.

[171] J. Boisvert, C. Shu, S. Wuhrer, and P. Xi, "Three-dimensional human shape inference from silhouettes: reconstruction and validation," Machine vision and applications, vol. 24, no. 1, pp. 145-157, 2013.

[172] L. Wang and A. Wong, "Covid-net: A tailored deep convolutional neural network design for detection of covid-19 cases from chest radiography images," arXiv preprint arXiv:2003.09871, 2020.

[173] F. Shi, J. Wang, J. Shi, Z. Wu, Q. Wang, Z. Tang, K. He, Y. Shi, and D. Shen, "Review of artificial intelligence techniques in imaging data acquisition, segmentation and diagnosis for covid-19," IEEE Reviews in Biomedical Engineering, 2020 .

[174] S. Karanam, R. Li, F. Yang, W. Hu, T. Chen, and Z. Wu, "Towards contactless patient positioning," IEEE Transactions on Medical Imaging, pp. 1-1, 2020. 